\title{
A DNS Study on the Effects of Streamwise Convex Surface Curvature on Coherent Structures in Turbulent Boundary Layers
}

by

Nikunj Dave

A thesis submitted to the Faculty of Graduate and Postdoctoral Affairs in partial fulfillment of the requirements for the degree of

\author{
Master of Applied Science \\ in \\ Aerospace Engineering
}

Carleton University

Ottawa, Ontario

(C) 2012, Nikunj Dave 
Library and Archives

Canada

Published Heritage

Branch

395 Wellington Street

Ottawa ON K1A ON4

Canada
Bibliothèque et

Archives Canada

Direction du

Patrimoine de l'édition

395 , rue Wellington

Ottawa ON K1A ON4

Canada
Your file Votre référence

ISBN: 978-0-494-93488-3

Our file Notre référence

ISBN: $978-0-494-93488-3$
NOTICE:

The author has granted a nonexclusive license allowing Library and Archives Canada to reproduce, publish, archive, preserve, conserve, communicate to the public by telecommunication or on the Internet, loan, distrbute and sell theses worldwide, for commercial or noncommercial purposes, in microform, paper, electronic and/or any other formats.

The author retains copyright ownership and moral rights in this thesis. Neither the thesis nor substantial extracts from it may be printed or otherwise reproduced without the author's permission.
AVIS:

L'auteur a accordé une licence non exclusive permettant à la Bibliothèque et Archives Canada de reproduire, publier, archiver, sauvegarder, conserver, transmettre au public par télécommunication ou par l'Internet, prêter, distribuer et vendre des thèses partout dans le monde, à des fins commerciales ou autres, sur support microforme, papier, électronique et/ou autres formats.

L'auteur conserve la propriété du droit d'auteur et des droits moraux qui protege cette thèse. $\mathrm{Ni}$ la thèse ni des extraits substantiels de celle-ci ne doivent être imprimés ou autrement reproduits sans son autorisation.
In compliance with the Canadian Privacy Act some supporting forms may have been removed from this thesis.

While these forms may be included in the document page count, their removal does not represent any loss of content from the thesis.
Conformément à la loi canadienne sur la protection de la vie privée, quelques formulaires secondaires ont été enlevés de cette thèse.

Bien que ces formulaires aient inclus dans la pagination, il n'y aura aucun contenu manquant. 


\section{Abstract}

Direct numerical simulation results are used to establish the effect of streamwise convex surface curvature on the development of turbulent boundary layers, and the effect of such curvature on the forced-convection heat transfer variations observed at certain supercritical thermodynamic states. The results illustrate the stabilizing effects of this flow geometry through modification of the structure and distribution of hairpin-like vortical flow structures in the boundary layer. Specifically, the radial equilibrium mechanism existing on the streamwise convex surface acts to decrease the streamwise spacing of hairpin-like structures within a wave packet and increase the spanwise spacing of the hairpin-like structures in comparison to the behaviour of such structures within the boundary layer on a flat surface. Furthermore, enhancement of convective heat transfer realized at a particular heat flux-to-mass flux ratio with the working fluid at a supercritical thermodynamic state is observed to be reduced by the stabilizing effect of convex surface curvature. 


\section{Acknowledgements}

I would like to thank my thesis supervisor Dr. Metin I. Yaras, firstly, for providing me with this wonderful opportunity to work in an area of my interest and secondly, for his constant positive participation and encouragement in completing my research. Dr. Yaras' undying interest in the subject and passion for research motivated me in bringing this study to fruition.

My greatest thanks go to my family; my father Harish Dave, my mother Daksha Dave and my sister Kruti Dave for their perpetual love, encouragement and support throughout my years of study, especially while completing my Master's degree. This accomplishment would not have been possible without them.

The funding provided by Atomic Energy of Canada Limited is also gratefully acknowledged. 


\section{Table of Contents}

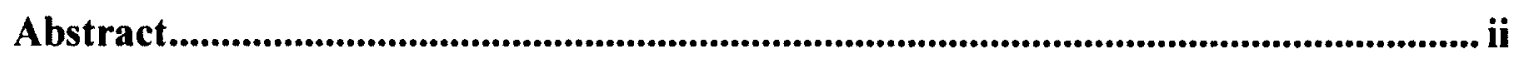

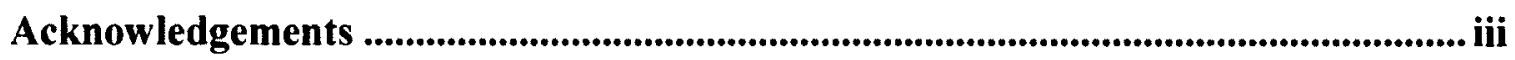

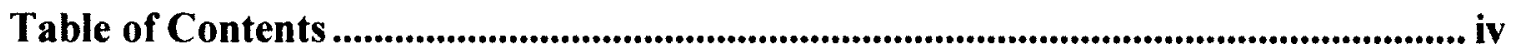

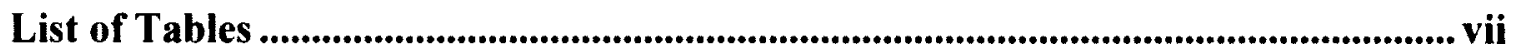

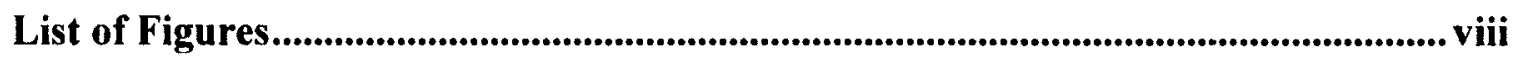

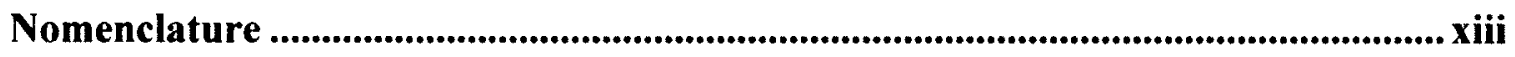

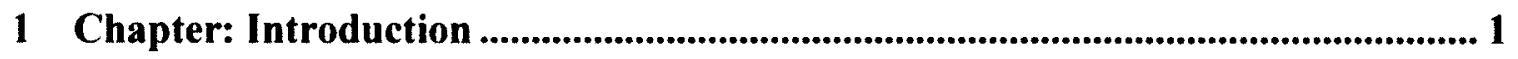

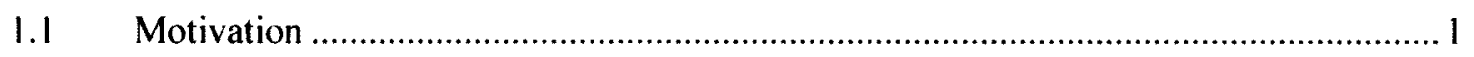

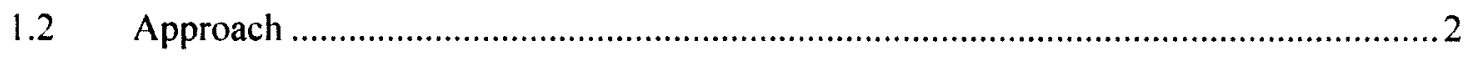

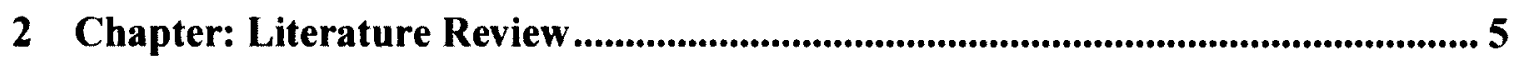

2.1 Forced Convection Heat Transfer in Supercritical Water .........................................5

2.2 Thermalhydraulics of Rod Bundles with Spiral Wire-Wrap .................................... 8

2.3 Coherent Flow Structures in Turbulent Boundary Layers...................................... 16

2.4 Effect of Streamwise Convex Surface Curvature on Turbulent Boundary Layers.......25

2.4.1 Typical Experimental Arrangement in the Study of Convex Surface Curvature

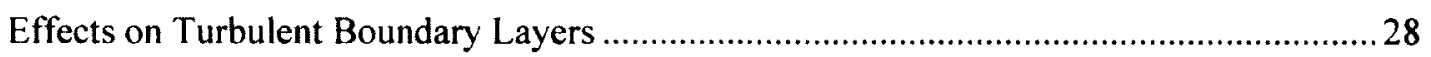

2.4.2 Effect of Streamwise Convex Surface Curvature on the Mean Turbulent Velocity

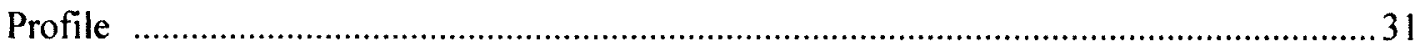

2.4.3 Effect of Streamwise Convex Surface Curvature on the Turbulent Boundary Layer..

2.4.3.1 Effect of Mildly-Curved Convex Surfaces on Turbulent Boundary Layers....34

2.4.3.2 Effects of Strongly-Curved Convex Surfaces on Turbulent Boundary Layers37

2.4.4 Effect of Streamwise Convex Surface Curvature on Convection Heat Transfer ..... 38 


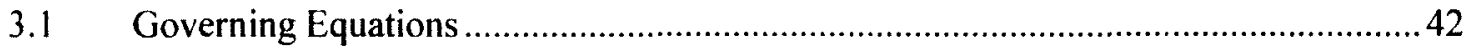

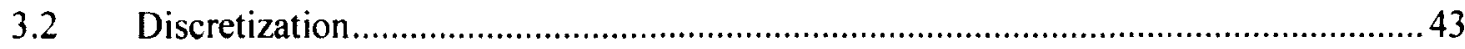

3.2.1 Convection and Diffusion Terms .................................................................47

3.2.2 The Coupled Solver........................................................................................48

3.3 Computational Method …………………….................................................. 50

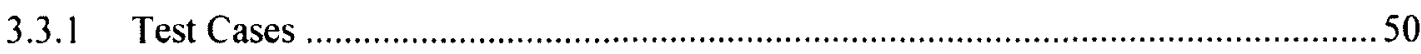

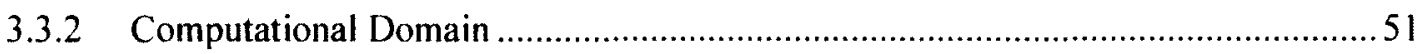

3.3.2.1 Computational Domain for the Baseline Flat-Surface Case..............................53

3.3.2.2 Computational Domain for the Streamwise Convex Surface Curvature Cases...

3.3.3 Spatial and Temporal Grid Resolution ...............................................................5

3.3.3.1 Spatial and Temporal Resolution for the Baseline Flat-Surface Case..............56

3.3.3.2 Spatial and Temporal Resolution for the Convex-Surface Cases....................57

3.3.4 Initial and Boundary Conditions …………………..........................................5

3.3.5 Forced Boundary Layer Disturbances.................................................................6

4 Chapter: Results and Discussion ................................................................................. 62

4.1 Validation of the Numerical Method......................................................................63

4.2 Effect of Streamwise Convex Surface Curvature on the Initial Evolution of Forced

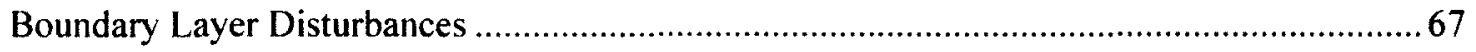

4.2.1 Evolution of the Initial Disturbance on the Mildly-Curved Streamwise Convex

Surface

4.2.2 Comparison of Evolution of the Initial Disturbance on the Mildly-Curved

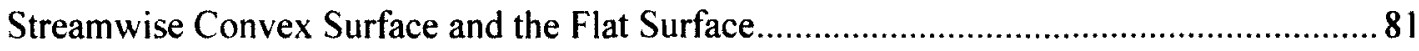

4.3 Effect of Streamwise Convex Surface Curvature on Turbulence in the Boundary Layer 
4.4 Effect of Streamwise Convex Surface Curvature on Forced Convection Heat Transfer .

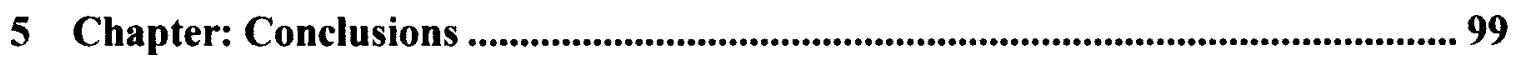

6 Chapter: Recommendations for Future Work............................................. 101

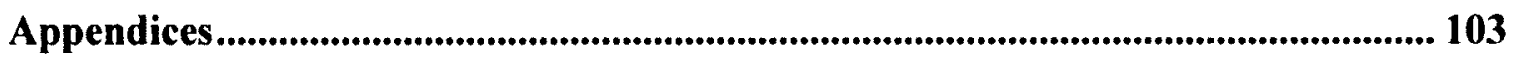

Appendix A : Review of Boundary-Layer Transition, Statistical Treatment of a Turbulent

Boundary Layer and Effect of Convex Surface Curvature on Transition. ............................ 103

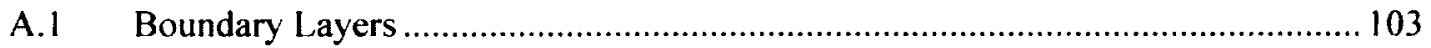

A.2 Effect of Streamwise Convex Surface Curvature on Shear-Layer Transition ....... 111

Appendix B : Effect of Mildly-Curved and Strongly-Curved Streamwise Convex Surface on an

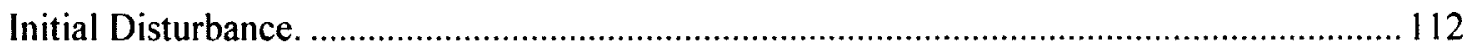

B.1 Evolution of an Initial Disturbance on a Strongly-Curved Streamwise Convex

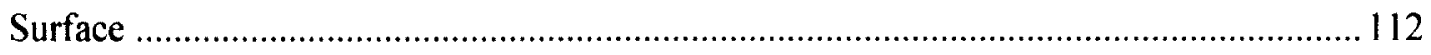

B.2 Comparison of Coherent Structures in the Turbulent Boundary Layer on a Mildly-

Curved Convex Surface Disturbed using Two Disturbance Duration Ranges.................. 118

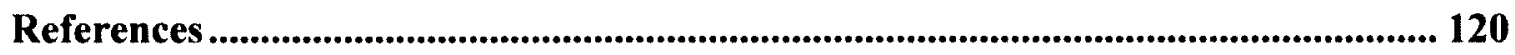




\section{List of Tables}

Table 2.1: Research conducted using wire-wrapped fuel rods.................................. 11

Table 2.2: Experiments conducted using convex surface curvatures. ...................... 30

Table 3.1: Simulation parameters. ...............................................................................51

Table 3.2: Computational domain parameters. ....................................................52

Table 3.3: Computational spatial grid resolution in the finely-resolved region. ...... 57

Table 3.4: Comparison of mean velocity profile data for various spatial grid

resolutions for a flat-surface simulation by Azih et al. (2012), ..................................58

Table 4.1: Nusselt number values and streak spacings. .............................................. 95

Table A.1: Boundary layer division based on mean velocity profile (Pope, 2000).. 109 


\section{List of Figures}

Figure 2.1: Property variations of supercritical water near the pseudo-critical temperature at a pressure of $24 \mathrm{MPa}$ (adapted from Shang and Lo, 2010)................. 6 Figure 2.2: A 7-pin wire-wrapped fuel-rod sub-assembly (adapted from Ahmad and Kim, 2006). $D$ - diameter of the fuel rod, $D_{w}$ - diameter of the spiral wire-wrap, $P$ spacing between adjacent fuel rods, $H$-pitch of the wire-wrap.................................... 9

Figure 2.3: Sub-channels within the fuel-rod assembly (adapted from Ahmad and Kim, 2006) 10

Figure 2.4: A 7-pin fuel-rod sub-assembly showing the induced motion of the flow due to the presence of wire-wrap spacers.

Figure 2.5: Evolution of a symmetric hairpin vortex in a shear flow: (a) initial distortion, (b) development of vortex legs and head, (c) evolution of subsidiary vortices and penetration toward the surface (adapted from Smith et al., 1991)....... 18 Figure 2.6: Schematic of a hairpin vortex attached to the wall (reproduced from Adrian et al., 2000).

Figure 2.7: Quadrants of the $u^{\prime} v^{\prime}$ plane indicating sweep and ejection events (adapted from Robinson, 1991).

Figure 2.8: Hairpin vortices inducing low-speed streaks: (a) symmetric case with wide separation of legs, (b) symmetric case with narrow leg separation, (c) asymmetric case (reproduced from Smith et al., 1991) 21

Figure 2.9: Hairpin wave-packet (reproduced from Zhou et al., 1999)....................... 23 
Figure 2.10: Hairpin vortex signature in the streamwise-wall-normal plane (reproduced from Adrian et al., 2000)

Figure 2.11: Experimental arrangement (adapted from Patel and Sotiropoulos, 1997). 28

Figure 2.12: Mean velocity profile on a convex surface (adapted from Ramaprian and Shivaprasad, 1977). The symbols represent different measuring stations along the curved surface with the difference from the flat-surface law of the wall increasing with distance along the curved surface. 33

Figure 3.1: Finite volume representation (adapted from ANSYS, 2009)................. 44

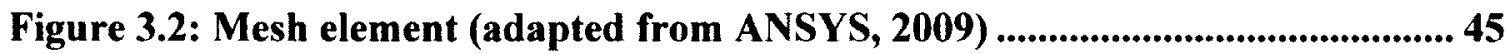

Figure 3.3: Schematic of the computational domain shown with a geometry

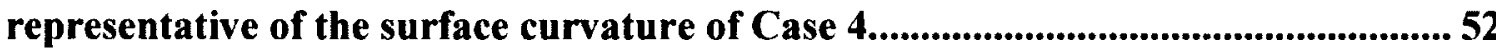

Figure 4.1: Comparison of mean velocity profiles for Case 1 with the flat-surface DNS data of Spalart (1988) and Wu and Moin (2009). 65

Figure 4.2: Comparison of fluctuating velocity fields for Case 1 with the flat-surface DNS data of Spalart (1988) and Wu and Moin (2010). 66

Figure 4.3: Comparison of production and dissipation rates from the budget of the Reynolds normal stresses for Case 1 with the flat-surface DNS data of Spalart (1988). 66

Figure 4.4: Shear layer roll-up to form an initial structure resembling a hairpin vortex loop at $t^{+}=12.5$. 
Figure 4.5: Stretching of the initial structure forming the head and legs of the vortex at $t^{+}=\mathbf{2 5}$. 73

Figure 4.6: Fluid motion induced by the counter-rotating legs shown with the perturbation velocity vector in a spanwise-wall-normal plane at $t^{+}=25$. The full domain is not shown for clarity. 74

Figure 4.7: Formation of shear layer with spanwise oriented vorticity above the hairpin vortex at $t^{+}=25$. The full domain is not shown for clarity. 74

Figure 4.8: Evolution of the disturbance: (a) formation of the secondary structure $B$; (b) formation of the tertiary structure $C$. 75

Figure 4.9: Formation of streamwise vortices consisting of vorticity of the opposite sign corresponding to each of the hairpin legs at $t^{+}=50$. The arrows indicate the fluid motion induced by the vortices. The streamwise vorticity contour level is selected to

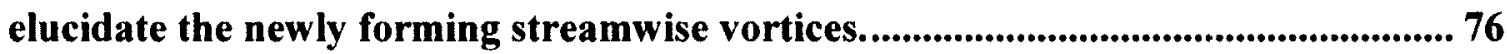

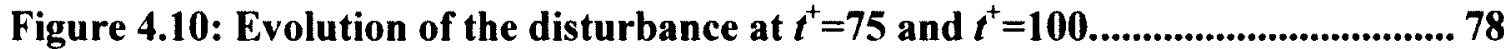

Figure 4.11: Shear layer with spanwise oriented vorticity at $t^{+}=62.5$ that rolls up to form structure $\mathbf{E}$ 79

Figure 4.12: Hairpin-like vortical structures at $t^{+}=125$ with the flow disturbance deactivated at $t^{+}=100$. The full domain is not shown for clarity. 80

Figure 4.13: Hairpin vortices at $t^{+}=200$ showing the dissipating vortices that were formed while the disturbance was active. The full domain is not shown for clarity. 81 Figure 4.14: Evolution of disturbance compared at $t^{+}=25$ : (a) flat surface (Case 1); (b) convex surface (Case 2) 82 
Figure 4.15: Evolution of disturbance compared at $t^{+}=125$ : (a) flat surface (Case 1);

(b) convex surface (Case 2)

Figure 4.16: Evolution of disturbance compared at $t^{+}=250$ : (a) flat surface (Case 1);

(b) convex surface (Case 2)

Figure 4.17: Evolution of disturbance compared at $t^{+}=400$ : (a) flat surface (Case 1);

(b) convex surface (Case 2). 85

Figure 4.18: Evolution of disturbance compared at $t^{+}=600$ : (a) flat surface (Case 1);

(b) convex surface (Case 2). 86

Figure 4.19: Iso-surfaces of $Q^{+}=\mathbf{0 . 0 0 2 7}$ showing hairpin-like structures in a fully turbulent boundary layer for (a) flat surface (Case 1); (b) convex surface (Case 2).87 Figure 4.20: Comparison of mean velocity profiles for flat-surface (Case 1) and convex-surface (Case 2).

Figure 4.21: Comparison of fluctuating velocity fields for flat-surface (Case 1) and convex-surface (Case 2).

Figure 4.22: Streamwise spacing of hairpin vortices in a wave packet in the nearwall region for (a) Case 1; (b) Case 2 visualized through flood plots of spanwise component of vorticity

Figure 4.23: Streamwise spacing of hairpin vortices in a wave packet further away from the wall for (a) Case 1; (b) Case 2 visualized through flood plots of spanwise component of vorticity

Figure 4.24: Spanwise streak spacing observed in the distribution of streamwise component of fluctuation velocity for (a) Case 1; (b) Case 2. 
Figure 4.25: Spanwise streak spacing observed in the distribution of streamwise component of fluctuation velocity for (a) Case 3; (b) Case 4........................................ 96 Figure 4.26: Mean rates of turbulence kinetic energy production and dissipation for Cases 1 and 2 (black lines), Case 3, Case 4 and data of Azih et al. (2012) (red lines).

Figure A.1: Process of transition from laminar to turbulent flow (adapted from Kachanov, 1994). 104

Figure A.2: Roadmap to turbulence (adapted from Saric et al., 2002)...................... 106

Figure B.1: Initial disturbance on the (a) flat surface and (b) convex surface. 113 Figure B.2: Evolution of the initial disturbance compared at $t^{\mathrm{n}}=1.5$ : (a) flat surface and (b) convex surface. 115

Figure B.3: Evolution of the initial disturbance compared at $t^{n}=3$ : (a) flat surface and (b) convex surface. 116 Figure B.4: Evolution of the initial disturbance compared at $t^{n}=5$ : (a) flat surface and (b) convex surface. 117 Figure B.5: Streamwise spacing of hairpin vortices in a wave packet for (a) Case 21; (b) Case 2-2 visualized through flood plots of spanwise component of vorticity.119 Figure B.6: Spanwise streak spacing observed in the distribution of streamwise component of fluctuation velocity for (a) Case 2-1; (b) Case 2-2. 119 


\section{Nomenclature}

$A$

Coefficient of the left-hand side of linearized Navier-Stokes equations

$b$

Coefficient of the right-hand side of linearized Navier-Stokes equations

$C_{p}$ Isobaric specific heat capacity $(\mathrm{J} / \mathrm{kg} \mathrm{K})$

$C_{f}$

Skin friction coefficient

$D$

Diameter of a nuclear-fuel rod $(\mathrm{m})$

$D_{w} \quad$ Diameter of a wire-wrap spacer $(\mathrm{m})$

$G \quad$ Mass flux $\left(\mathrm{kg} / \mathrm{m}^{2} \mathrm{~s}\right)$

$H \quad$ Pitch of a wire-wrap spacer (m)

$h_{0} \quad$ Total enthalpy $(\mathrm{J} / \mathrm{kg})$

$h$

Convection heat transfer coefficient $\left(\mathrm{W} / \mathrm{m}^{2} \mathrm{~K}\right)$

$k$

Thermal conductivity $(\mathrm{W} / \mathrm{m} \mathrm{K})$

$L \quad$ Length (m)

$l$

Apparent mixing length

$\dot{m}$

Mass flow rate $(\mathrm{kg} / \mathrm{s})$

$N \quad$ Number of grid nodes

$\mathrm{Nu} \quad$ Nusselt number

$P \quad$ Rate of turbulence kinetic production $\left(\mathrm{m}^{2} / \mathrm{s}^{3}\right)$

$p \quad$ Pressure $(\mathrm{Pa})$

$\operatorname{Pr} \quad$ Prandtl number

$Q \quad$ Second invariant of the velocity gradient tensor $\left(\mathrm{s}^{-2}\right)$

$q \quad$ Heat flux $\left(\mathrm{J} / \mathrm{m}^{2} \mathrm{~s}\right)$ 


\begin{tabular}{|c|c|}
\hline$R$ & Radius of curvature $(\mathrm{m})$ \\
\hline$R_{i j}$ & Reynolds stress tensor \\
\hline$r, \theta, z$ & $\begin{array}{l}\text { Radial }(m) \text {, angular (rad) and spanwise }(m) \text { coordinate directions in the } \\
\text { cylindrical coordinate system }\end{array}$ \\
\hline$R i$ & Richardson number \\
\hline $\operatorname{Re}$ & Reynolds number \\
\hline$r^{k}$ & Residual from the solution of the linearized Navier-Stokes equations \\
\hline St & Stanton number \\
\hline$T$ & Temperature $(\mathrm{K})$ \\
\hline$t$ & Time (s) \\
\hline$t_{k}$ & Kolmogorov time scale \\
\hline$u_{i}$ & Instantaneous velocity vector $(\mathrm{m} / \mathrm{s})$ \\
\hline $\bar{u}_{i}$ & Mean velocity vector $(\mathrm{m} / \mathrm{s})$ \\
\hline$u, v, w$ & Streamwise, wall-normal and spanwise velocity components $(\mathrm{m} / \mathrm{s})$ \\
\hline$u_{\tau}$ & Friction velocity $(\mathrm{m} / \mathrm{s})$ \\
\hline$V$ & Volume $\left(\mathrm{m}^{3}\right)$ \\
\hline$V_{\text {grad tensor }}$ & Velocity gradient tensor \\
\hline$V_{i j}$ & Row $(i)$ and column $(j)$ entry in the velocity gradient tensor $(i, j=1,2,3)$ \\
\hline$V_{\theta}, V_{r}, V_{z}$ & Velocity components in the $\theta, r, z$ cylindrical coordinate system $(\mathrm{m} / \mathrm{s})$ \\
\hline$x, y, z$ & Streamwise, wall-normal and spanwise coordinates $(\mathrm{m})$ \\
\hline
\end{tabular}




$\begin{array}{ll}\text { Greek letters } & \\ \delta & \text { Boundary layer thickness }(\mathrm{m}) \\ \delta_{v} & \text { Viscous length scale }(\mathrm{m}) \\ \varepsilon & \text { Rate of turbulence kinetic dissipation }\left(\mathrm{m}^{2} / \mathrm{s}^{3}\right) \\ \eta & \text { Kolmogorov length scale }(\mathrm{m}) \\ \theta & \text { Momentum thickness }(\mathrm{m}) \\ \kappa & \text { von Kármán constant }(=0.41) \\ \mu & \text { Dynamic viscosity }(\mathrm{kg} / \mathrm{m} \mathrm{s}) \\ \nu & \text { Kinematic viscosity }\left(\mathrm{m}^{2} / \mathrm{s}\right) \\ \rho & \text { Density }\left(\mathrm{kg} / \mathrm{m}^{3}\right) \\ \tau_{i j} & \text { Viscous stress tensor }\left(\mathrm{N} / \mathrm{m}^{2}\right) \\ \tau & \text { Shear stress }\left(\mathrm{N} / \mathrm{m}^{2}\right) \\ \varphi & \text { Generic variable } \\ \Omega & \text { Vorticity }\left(\mathrm{s}^{-1}\right)\end{array}$

\section{Subscripts}

$b$

Bulk/freestream

$f$

Film

$f r$

Finely-resolved region

$i$

Control-volume or node-number index

ip

Integration points

$i, j \quad$ Tensor notation. $i, j=1,2,3$ indicates streamwise, wall-normal and spanwise coordinate directions, respectively. 


$\begin{array}{ll}\text { momentum } & \text { Momentum boundary layer } \\ p c & \text { Pseudo-critical } \\ r m s & \text { Root mean square } \\ \text { thermal } & \text { Thermal boundary layer } \\ u & \text { Streamwise location of transition to turbulence } \\ u p & \text { Upstream node } \\ w & \text { Wall } \\ x & \text { Streamwise component } \\ y & \text { Wall-normal component } \\ z & \text { Spanwise component } \\ \eta & \text { Kolmogorov scale } \\ \theta & \text { Momentum thickness } \\ \tau & \text { Friction } \\ \infty & \text { Freestream conditions }\end{array}$

\section{Superscripts}

$\begin{array}{ll}+ & \text { Normalization by wall variables (friction velocity and kinematic viscosity) } \\ & \text { Fluctuation in flow-property } \\ n b & \text { Neighbouring nodes } \\ \text { T } & \text { Matrix transpose }\end{array}$




\section{Acronyms}

$\mathrm{AC}$

Additive Correction

AECL Atomic Energy of Canada Limited

$\begin{array}{ll}\text { AMG } & \text { Algebraic multigrid } \\ \text { CANDU } & \text { Canada Deuterium Uranium } \\ \text { CFD } & \text { Computational fluid dynamics } \\ \text { DHT } & \text { Deteriorated heat transfer } \\ \text { DHV } & \text { Downstream hairpin vortex } \\ \text { DNS } & \text { Direct numerical simulation } \\ \text { GIF } & \text { Generation IV International Forum } \\ \text { IAPWS } & \text { International Association for the Properties of Water and Steam } \\ \text { IHT } & \text { Improved heat transfer } \\ \text { THU } & \text { Tollmien-Schlichting } \\ \text { SHV } & \text { Incomplete Lower Upper } \\ \text { RHV } & \text { Particle image velocimetry } \\ \text { LES } & \text { Large eddy simulation } \\ \text { NHT } & \text { Normal heat transfer } \\ \text { N-S } & \text { Navier-Stokes } \\ \end{array}$




\section{Chapter: Introduction}

\subsection{Motivation}

Atomic Energy of Canada Limited (AECL) is developing the Canada Deuterium Uranium (CANDU) supercritical water reactor (SCWR) in collaboration with the Generation IV International Forum (GIF) which identified the SCWR as one of the next generation of evolutionary nuclear energy concepts (Torgerson et al., 2006). The SCWR uses water at the supercritical thermodynamic state as coolant for the nuclear fuel rods. The increased temperature range of the power cycle and a lack of phase change of water at the supercritical thermodynamic state provide a higher thermal efficiency and a more compact overall design compared to conventional nuclear reactors (Roelofs, 2004). Such advantages have led to increased research on the thermalhydraulics of the SCWR by GIF member nations (Pioro and Duffey, 2005). One of several such areas of research proposed for the SCWR is the examination of the effect of wire-wrap spacers on heat transfer from the fuel rods to the supercritical coolant in the fuel-bundle. The wire-wrap spacers wound helically around each of the fuel rods serve to maintain the spatial positioning of the rods within the fuel bundle and affect the turbulent coolant flow and heat transfer processes (Natesan et al., 2010). The wire-wrap spacers simultaneously induce transverse velocities and impose streamwise convex curvature on the turbulent coolant flow as it develops over the surface of each fuel rod in the bundle; establishing the effect of such streamwise convex curvature on convective heat transfer affected by flow turbulence is the motivation for the present study. 


\subsection{Approach}

Experiments (Head and Bandyopadhyay, 1981; Adrian et al., 2000) and numerical simulations (Zhou et al., 1999; Wu and Moin, 2009) of turbulent flows have shown the existence of coherent flow structures of turbulence which are believed to dominate the wall-normal momentum and energy transfer processes in the boundary layer (for example. Buschmann et al., 2009). Therefore, the study of turbulent boundary layers in terms of the coherent flow structures of turbulence can be expected to provide an improved understanding of the momentum and heat transfer processes occurring in the boundary layer. However, turbulence is a complex process involving varying degrees of spatial and temporal scales of coherent structures in the flow and thus, capturing all relative scales in the flow can only be achieved with carefully set up experiments or direct numerical simulations. The present study utilizes direct numerical simulations to study the behaviour and interactions of coherent structures in fully turbulent boundary layers.

The objective of the present research is to characterize the effect of streamwise convex surface curvature which is induced by the wire-wrap spacers on the turbulent flow of water at a supercritical thermodynamic state. Experiments with surface curvature have shown that streamwise convex surface curvature exerts a stabilizing influence on turbulent boundary layers, resulting in decreased skin friction coefficients and Reynolds stresses compared to similar flows over flat surfaces (for example, So and Mellor, 1973; Meroney and Bradshaw, 1975; Ramaprian and Shivaprasad, 1978; Gillis and Johnston, 1983). The experimentally-observed stabilizing effect of convex surface curvature suggests that there are changes in the development of coherent flow structures in the 
boundary layer. In the present study, the effect of streamwise convex surface curvature on the coherent flow structures of turbulence is first established with the use of air as the working fluid at standard atmospheric pressure and temperature without any wall-normal fluid property variations, in comparison with the development of the coherent flow structures in a similar flow environment on a flat surface. This is accomplished because experiments with water at the supercritical thermodynamic state have shown that at certain temperatures above the critical pressure, convective heat transfer is significantly affected by thermophysical property variations of supercritical water (Pioro et al., 2004). In particular, recent direct numerical simulations by the author's research group (Azih et al.. 2012) established that for certain heat flux and mass flux conditions with the working fluid at a supercritical thermodynamic state, the coherent flow structures of turbulence are modified yielding variations in the wall-normal mass and energy transport due to turbulence. Thus, after characterizing the effect of convex surface curvature on the coherent flow structures in the absence of wall-normal fluid property variations, water at the supercritical thermodynamic state is employed as the working fluid to elucidate the effect of convex surface curvature on convective heat transfer within the boundary layer in the presence of thermophysical property variations.

It should be noted that although the wire-wrap spacers induce streamwise convex curvature in sub-channels within the fuel-rod bundles, the present study addresses the effect of streamwise convex surface curvature on coherent flow structures in boundary layers. Several studies have determined that large Reynolds stresses associated with the production of turbulence occurs in the region close to the walls (for example, Bernard et al., 1993; Brooke and Hanratty, 1993). The production of Reynolds stresses is strongly 
linked to organized motion of the coherent flow structures in the near-wall region and the behaviour of such structures is not significantly affected by the outer flow (Brooke and Hanratty, 1993). Therefore, a study of the development of coherent flow structures in the near-wall region of a streamwise convex surface in a boundary layer should provide physical insights into flows developing in fuel-rod bundle sub-channels. 


\section{Chapter: Literature Review}

This chapter begins with a review on the effect of the supercritical thermodynamic state of water on heat transfer followed by a review on the effect of wirewrap spacers on fuel rods in nuclear fuel bundles. Next, an overview of the concepts and theories used to understand the structure of turbulent shear layer flows is presented followed by a review of the investigations conducted on the effect of streamwise convex surface curvature on such flows.

\subsection{Forced Convection Heat Transfer in Supercritical Water}

GIF has proposed the development of the SCWR which is an advanced nuclear reactor concept that uses supercritical water as coolant. Supercritical water refers to a state that is above the thermodynamic critical point of water at a temperature of 647.096 Kelvin $(\mathrm{K})$ and a pressure of $22.064 \mathrm{MPa}$. The use of water at the supercritical thermodynamic state as a coolant provides a higher thermal efficiency and a more compact overall design for the SCWR (Roelofs, 2004). An important property of supercritical water as shown by experiments is that heat transfer is significantly affected by thermophysical property variations near the thermodynamic critical and pseudocritical points. Pseudo-critical points in state space are defined as points above the critical pressure where the specific heat has a maximum value at particular temperatures (Pioro et al., 2004). An example of thermophysical property variations at a pressure of $24 \mathrm{MPa}$ based on the data published by the International Association for the Properties of Water and Steam (IAPWS) is shown in Figure 2.1. 


\section{Molecular viscosity (Pa s) \\ Specific heat capacity $(\mathrm{kJ} / \mathrm{kg} \mathrm{K})$ \\ Thermal conductivity $(\mathrm{W} / \mathrm{m} \mathrm{K})$ \\ Density $\left(\mathrm{kg} / \mathrm{m}^{3}\right)$}

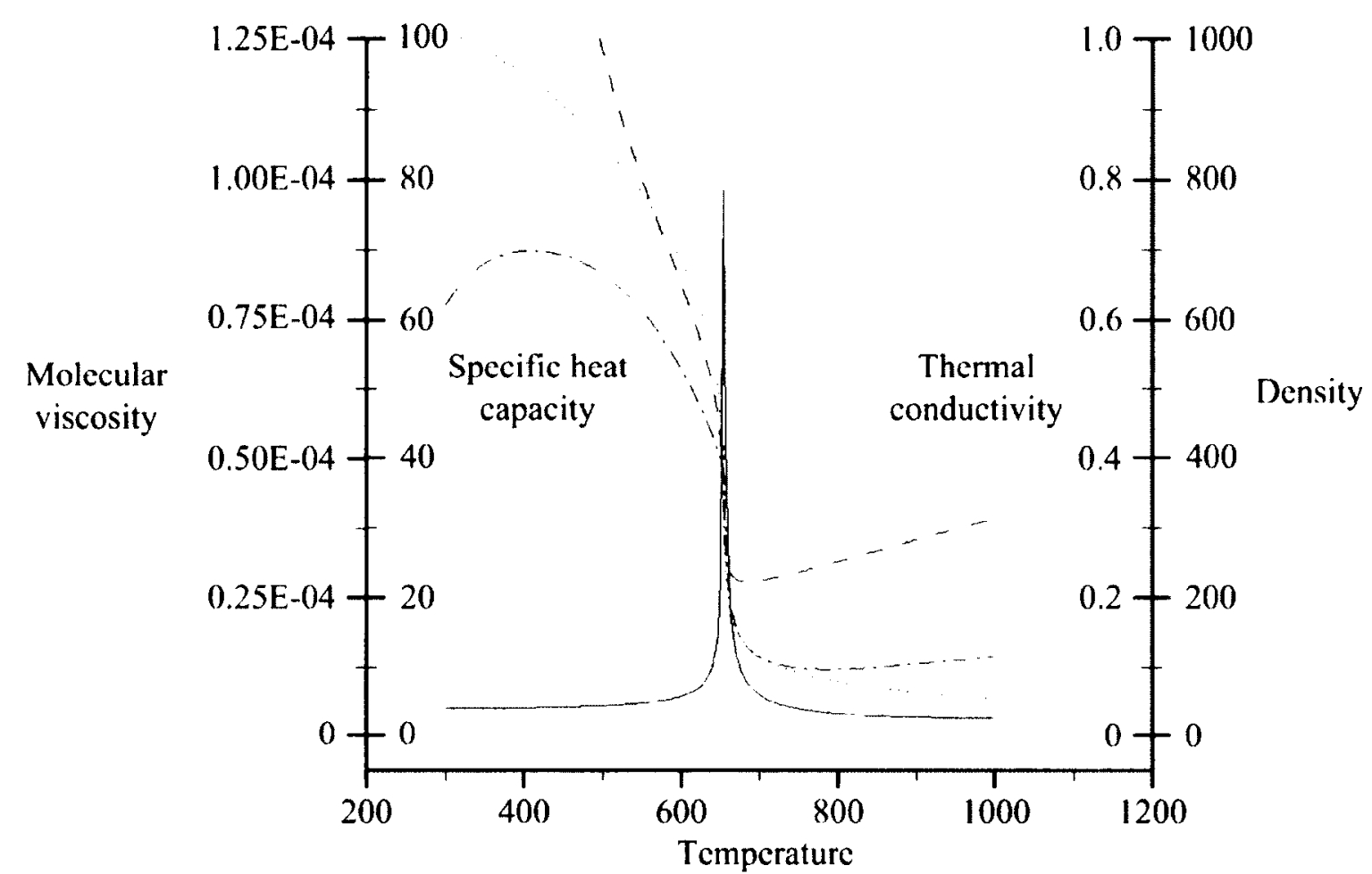

(K)

Figure 2.1: Property variations of supercritical water near the pseudo-critical temperature at a pressure of 24 MPa (adapted from Shang and Lo, 2010).

The application of supercritical water as coolant requires the knowledge of its heat transfer properties. However, empirical correlations such as the Dittus-Boelter model fail to accurately predict the convective heat transfer coefficient particularly in the vicinity of the pseudo-critical point (Pioro et al., 2004). The Dittus-Boelter correlation is expressed as:

$$
N u=k R e^{m} P r^{n}
$$


In this equation, $N u$ is the Nusselt number, $R e$ is the Reynolds number and $P r$ is the Prandtl number of the flow. The parameters $k, m, n$ are constants, which for subcritical single-phase flows are generally taken as $k=0.023, m=0.8$ and $n=0.4$ for heating and $n=0.3$ for cooling (Winterton, 1998). Due to the significant temperature dependence of the thermophysical properties, it is crucial that the temperature at which the fluid property values are evaluated in empirical relations is clearly identified by the researcher (McEligot and Jackson, 2004). When the observed heat transfer coefficient in supercritical flows is in agreement with the Dittus-Boelter equation, it is termed as normal heat transfer (NHT). Yamagata et al. (1972) mention that previous experimental investigations on forced convective heat transfer to supercritical water at low heat fluxes have shown an increase in the heat transfer coefficient with a maximum near the pseudocritical temperature. Hence, lower values of the wall temperature are observed in comparison to the NHT mode and this is known as improved heat transfer (IHT). However, as the heat flux is increased, the peak in the heat transfer coefficient decreases until a stage where lower heat transfer coefficient and a corresponding higher wall temperature is observed as compared to the NHT mode; this is known as deteriorated heat transfer (DHT) (Pioro and Duffey, 2005). The substantial thermophysical property variations in the vicinity of the pseudo-critical point (see Figure 2.1 , for example) are believed to cause these deviations in the heat transfer coefficient from that predicted by the Dittus-Boelter correlation.

In a numerical study using supercritical carbon dioxide, Bae et al. (2005) observe DHT in a region where turbulence is attenuated followed by enhancement in heat transfer when turbulence is restored. In order to obtain a better understanding of DHT and IHT 
mechanisms, it is therefore worthwhile to conduct a detailed study of the behaviour of the flow in terms of the turbulence flow structures. This is possible with the aid of carefully executed experiments and numerical simulations. Bae et al. (2005) mention that given the lack of uniformity between experimental results and numerical simulations performed with turbulence models, the use of direct numerical simulations (DNS) is recommended. DNS also provides an effective alternative to experiments especially for supercritical working fluids where the typically high pressures and temperatures require the use of expensive and complicated experimental arrangements (Gu et al., 2010).

\subsection{Thermalhydraulies of Rod Bundles with Spiral Wire-Wrap}

The heat energy production in a nuclear reactor core occurs due to nuclear reaction in bundles of nuclear-fuel rods present in the core. According to Natesan et al. (2010), the heat generation depends on the spacing between the rods in a bundle which is maintained by grid spacers or thin wires wound helically around the fuel rods. Grid spacers occupy less volume but the spiral wire-wrap arrangement is preferred as it is known to increase the turbulence level thereby enhancing the thermal mixing process (Natesan et al., 2010). The wire-wrap is also easier to manufacture and is less expensive than grid spacers (Natesan et al., 2010). The turbulent flow through a bare fuel bundle (without grid spacers or wire-wrapped fuel rods) shows a relatively uniform distribution of velocity and temperature at any given cross-section of the assembly without any significant mixing between sub-channels, and with the velocity and temperature gradients restricted to a small region close to the surface of the fuel rods (Sreenivasulu and Prasad, 2011). The turbulent transport of momentum and energy is mostly restricted to within the 
sub-channels. Figure 2.2 and Figure 2.3 show an example of a fuel-rod sub-assembly with the corresponding sub-channels. The result is reduced heat transfer between the fuel rods and the coolant which decreases thermal efficiency and increases the possibility of a certain section in the sub-assembly reaching higher temperatures and thus higher thermal stresses in comparison to the wire-wrapped fuel bundle. Experiments with wire-wrapped fuel rods have shown that the flow mixing between sub-channels is enhanced, which provides higher heat transfer rates (Peniguel et al., 2010). According to Ginsberg (1972), the presence of the wire-wrap spacer induces lateral components of velocity in the assembly thus inducing a net transport of fluid carrying momentum and energy from each sub-channel to adjacent sub-channels. Table 2.1 lists the experimental and numerical investigations conducted on wire-wrap spacers cited in this thesis with the relevant experimental parameters.
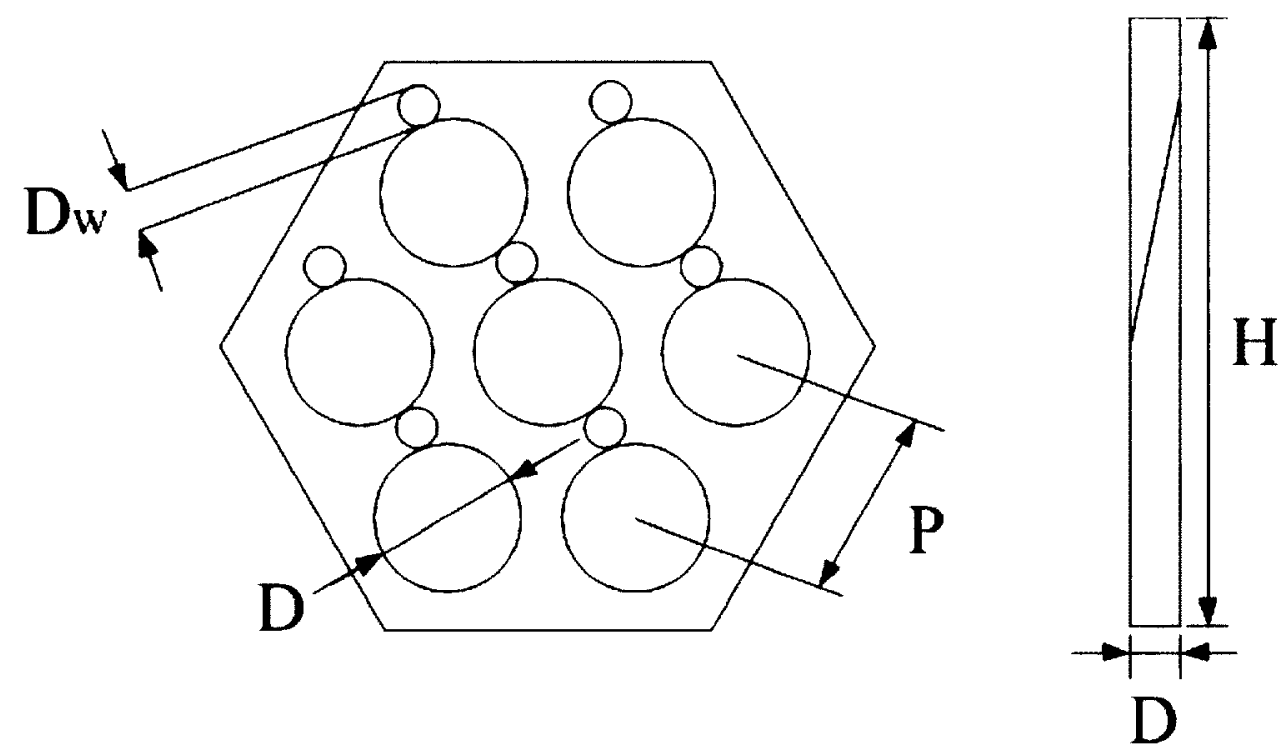

Figure 2.2: A 7-pin wire-wrapped fuel-rod sub-assembly (adapted from Ahmad and Kim, 2006). $D$ - diameter of the fuel rod, $D_{w}$ - diameter of the spiral wire-wrap, $P$ spacing between adjacent fuel rods, $H$-pitch of the wire-wrap. 


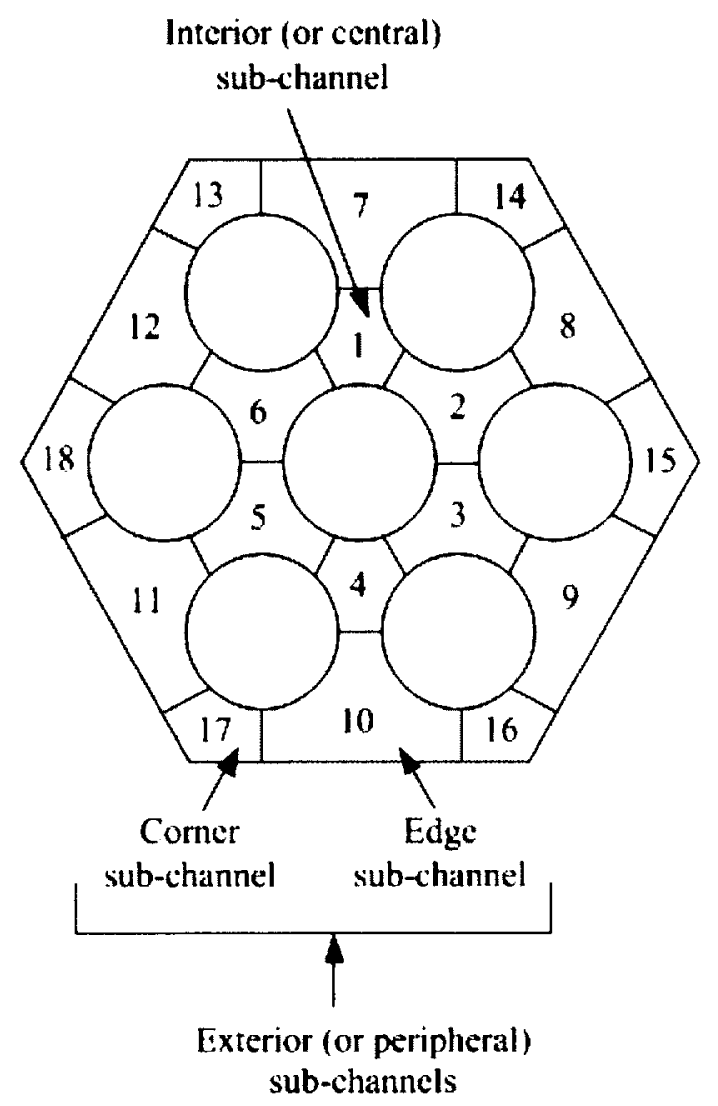

Figure 2.3: Sub-channels within the fuel-rod assembly (adapted from Ahmad and Kim, 2006). 
Table 2.1: Research conducted using wire-wrapped fuel rods.

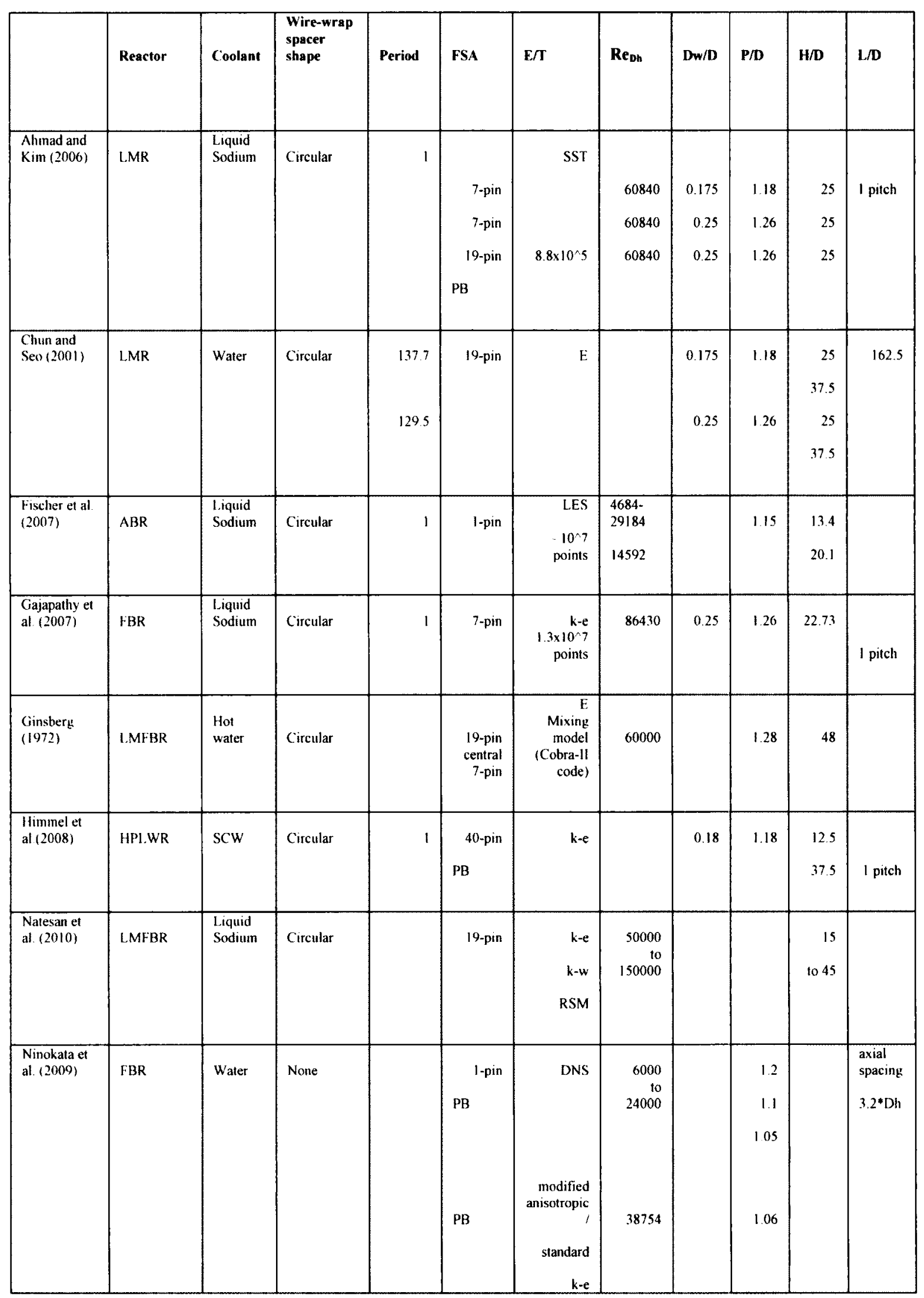




\begin{tabular}{|c|c|c|c|c|c|c|c|c|c|c|c|}
\hline & Reactor & Coolant & $\begin{array}{l}\text { Wire-wrap } \\
\text { spacer } \\
\text { shape }\end{array}$ & Period & FSA & $\mathbf{E} / \mathbf{T}$ & $\mathbf{R e}_{\mathrm{Dh}}$ & Dw/D & $\mathrm{P} / \mathrm{D}$ & H/D & $L / D$ \\
\hline $\begin{array}{l}\text { Raza and } \\
\text { Kinn (2008) }\end{array}$ & LMR & $\begin{array}{l}\text { Liquid } \\
\text { Sodium }\end{array}$ & $\begin{array}{l}\text { Circular, } \\
\text { hexagon } \\
\text { and } \\
\text { rhombus } \\
\text { Circular }\end{array}$ & 1 & $\begin{array}{l}\text { 7-pin } \\
\text { 7-pin } \\
\text { 19-pin } \\
\text { PB }\end{array}$ & $\begin{array}{r}\text { SST } \\
\text { SST } \\
9.46 \times 10^{\circ 5} \\
2.14 \times 10^{\circ} 6\end{array}$ & $\begin{array}{l}60000 \\
60000\end{array}$ & $\begin{array}{l}0.25 \\
0.25 \\
0.25\end{array}$ & $\begin{array}{l}1.26 \\
1.26 \\
1.26\end{array}$ & $\begin{array}{r}12.5 \\
25 \\
25\end{array}$ & I pitch \\
\hline $\begin{array}{l}\text { Rolfo et al. } \\
(2011)\end{array}$ & FBR & & Circular & 1 & $\begin{array}{l}\text { 7-pin } \\
\text { 19-pin, } \\
61-\text { pin } \\
\text { PB }\end{array}$ & $\begin{array}{l}k-\mathrm{e} \\
\text { SMC }\end{array}$ & & & 1.1 & $\begin{array}{l}21, \\
16.8\end{array}$ & I pitch \\
\hline $\begin{array}{l}\text { Sreenivasulu } \\
\text { and Prasad } \\
\text { (2011) }\end{array}$ & $\begin{array}{l}\text { Heat } \\
\text { Exchanger }\end{array}$ & Water & Circular & & 7-pin & k-w SST & & & $\begin{array}{l}1.28 \\
1.28 \\
1.28 \\
1.32 \\
1.36\end{array}$ & $\begin{array}{r}9.09 \\
18.18 \\
30.3\end{array}$ & \\
\hline
\end{tabular}

LMR - Liquid metal reactor, ABR - Advanced breeder reactor, LMFBR - Liquid metal fast breeder reactor, HPLWR - High performance light water reactor, FBR - Fast breeder reactor, SCW - Supercritical water, FSA - Fuel sub-assembly, PB - Periodic boundaries, E/T - Experiment/Turbulence Model, SST Shear stress transport, k-e - Turbulence kinetic energy-turbulence dissipation rate, k-w - Turbulence kinetic energy-turbulence specific dissipation rate, SMC - Second moment closure, RSM - Reynolds stress model, D - Diameter of the fuel rod, Dw - Diameter of the spiral wire-wrap, P - Spacing between adjacent fuel rods, $\mathrm{H}$ - Pitch of the wire-wrap, $\mathrm{L}$ - Length of the fuel rod, $\mathrm{Re}_{\mathrm{Dh}}$ - Reynolds number based on hydraulic diameter.

Many researchers have observed that the flow induced by the wire-wraps in the inner and outer regions of a sub-assembly is fairly independent of each other especially with an increase in the number of fuel rods (Rolfo et al., 2011). The outer region consists of the edge and corner sub-channels (peripheral or exterior sub-channels) and the inner 
region consists of the central (or interior) sub-channels as shown in Figure 2.3. Bishop and Todreas (1980) refer to the flow along the direction of the wire-wrap in the peripheral sub-channels as "sweeping" flow and that in the central sub-channels as "mixing" flow. The magnitude of the sweeping flow is greater than that of the mixing flow due to the presence of surrounding wire-wrapped rods in the central sub-channels (Bishop and Todreas, 1980; Gajapathy et al., 2007; Rolfo et al., 2011). In the central subchannels, while the wire-wrap on one rod brings flow into a particular sub-channel, the wire-wrap on an adjacent rod convects fluid out of that sub-channel (for example, see Figure 2.4). This leads to enhanced flow mixing in the central sub-channels as compared to the peripheral sub-channels (Sreenivasulu and Prasad, 2011). Due to these secondary motions induced by the wire-wrap, transverse pressure gradients are also created from one end of a cross-section to the other in a sub-assembly (Ahmad and Kim, 2006). Raza and Kim (2008) observe a region of recirculating flow behind the wire-wrap due to flow blockage in all sub-channels of a 7-rod bundle, but for a 19-rod bundle, such recirculating flows are only observed in the sub-channels close to the end-walls. This indicates that the flow mixes well in the central sub-channels of the 19-rod bundle. It also indicates that the flow in the peripheral sub-channels have an effect on the flow in the central sub-channels with this effect reducing with an increasing number of rods. Such flow mixing would also affect the temperature distribution in a sub-assembly within the nuclear fuel bundle. Ahmad and Kim (2006) determine that the wire-wrapped fuel-rod bundle reduces the temperature gradients and the maximum temperature as compared to the bare fuel bundle which also results in a higher local Nusselt number $(\mathrm{Nu})$ number for the wire-wrapped bundle. A similar result is reported by Gajapathy et al. (2009) who find the temperature 
difference between the peripheral and central sub-channels in a bundle with wirewrapped rods to be $1 / 4^{\text {th }}$ of that observed in a bare fuel bundle.

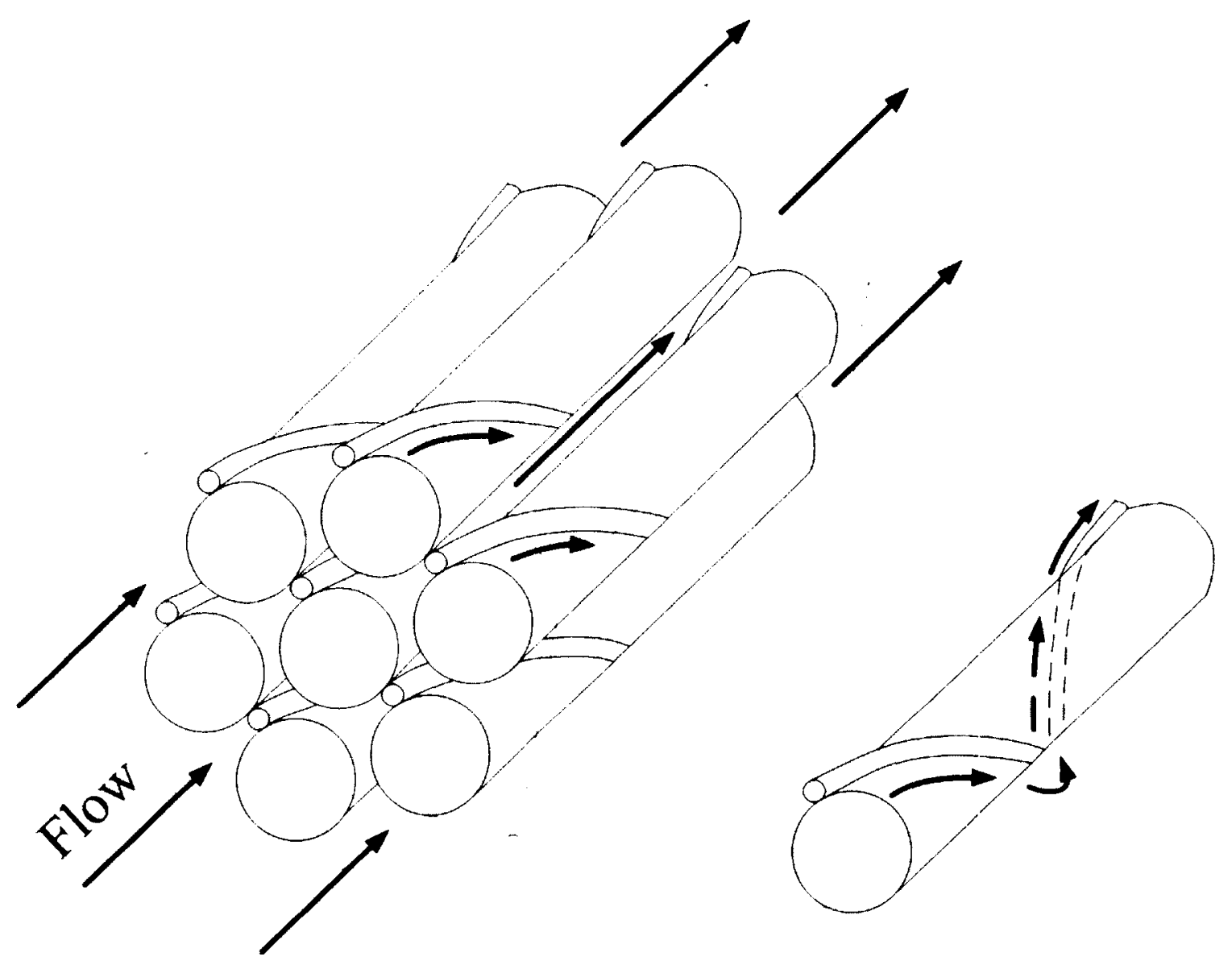

Figure 2.4: A 7-pin fuel-rod sub-assembly showing the induced motion of the flow due to the presence of wire-wrap spacers.

Based on the foregoing observations, there is ample evidence to indicate that the presence of the wire-wrap enhances flow mixing between sub-channels. However, the underlying physical mechanisms are poorly understood, which is important in the perspective of designing more effective techniques for improving the efficiency and safety of fuel-rod bundles with wire-wrapped rods. To improve the understanding of the flow through a fuel-rod bundle with wire-wrapped rods, a thermalhydraulic analysis of a fuel-rod bundle is preferred, but the thermalhydraulic analysis of even a single sub- 
assembly in a reactor is difficult due to the presence of several sub-channels (Fischer et al., 2007). Sub-channel modelling is often used for thermalhydraulic calculations as it is more efficient for design purposes (Ahmad and Kim, 2006). A substantial understanding of the flow physics is required for the development of reliable mathematical models to predict the thermalhydraulics of wire-wrapped fuel-rod bundles that capture the subchannel mixing induced by the wire-wraps, including the effects of the geometrical parameters of the wire-wrapped fuel-rod bundles such as the adjacent fuel-rod spacing to fuel-rod diameter ratio $(P / D)$, wire-wrap pitch to fuel-rod diameter ratio $(H / D)$, wire diameter and cross-sectional shape which are known to affect flow mixing (Himmel et al., 2008; Raza and Kim, 2008; Ninokata et al., 2009; Sreenivasulu and Prasad, 2011). Computational fluid dynamics (CFD), especially DNS or large eddy simulations (LES), provide a good alternative to experiments for understanding such flows. Although the use of DNS requires significant computing power when compared to the traditional Reynolds averaged Navier-Stokes (RANS) methods, RANS suffers from the shortcoming of turbulence closures that cannot accurately capture the flow features in a fuel-rod bundle with spiral wire-wraps (Ninokata and Merzari, 2008). The computing power required for DNS or LES can be reduced with a judicious choice of boundary conditions based on the expected behavior of the flow. In an LES of a single wire-wrapped rod in a hexagonally periodic cell performed by Fischer et al. (2007), they observe that the flow reaches a fully turbulent state within one pitch of the spiral wire-wrap. Such observations and distinct flow features in the inner and outer regions of a sub-assembly allow the selection of a particular central or peripheral sub-channel and conducting DNS or LES with periodic boundary conditions. 
Before conducting DNS of a sub-channel, it would be beneficial to investigate the effects of streamwise convex surface curvature on turbulence in isolation since the wirewrap spacers impose convex surface curvature on the turbulent coolant flow as it develops within the fuel bundle (see Figure 2.4). Such effects of curvature should be studied in terms of the development of the coherent flow structures of turbulence within the shear layer that are known to play an important role in the wall-normal turbulent mass and energy transport (Buschmann et al., 2009). Accordingly, the next two sections review shear layer turbulence in terms of the coherent flow structures and the effect of streamwise convex curvature on turbulence.

\subsection{Coherent Flow Structures in Turbulent Boundary Layers}

This section presents recent efforts directed at understanding the structure of turbulent boundary layer flows. Basic information regarding transition on boundary layer flows and the statistical treatment of turbulent boundary layers is presented in Appendix A.1. Flow visualization experiments such as those performed by Head and Bandyopadhyay (1981), Acarlar and Smith (1987b), Adrian et al. (2000) and direct numerical simulations (DNS) such as those performed by Zhou et al. (1999), Wu and Moin (2009) among many others have shown that transitional and turbulent shear layers are populated by coherent flow structures. Adrian (2007) suggests that only structures that persist for longer times to be captured in flow visualization techniques or significantly add to the time-averaged statistics should be referred to as coherent flow structures. The dominant flow structure within the turbulent boundary layer is the hairpin-like vortical flow structure (Smith et al., 1991). Theodorsen conceptualized this 
structure as a horseshoe vortex as far back as in 1952 but the topology of these hairpinlike vortical structures varies with flow parameters such as the Reynolds number. For example, Head and Bandyopadhyay (1981) observed that at lower momentum-thickness Reynolds numbers $\left(\operatorname{Re}_{\theta}<800\right)$, the vortices resembled a shape more similar to a horseshoe but as the Reynolds number increased, the vortices were elongated in the streamwise direction and resembled hairpins. Furthermore, at higher Reynolds numbers, the population of the hairpin-like vortical structures within the turbulent boundary layer increases, and the mutual interaction with increased instances of such vortices results in one-legged or cane-shaped vortices (Smith et al., 1991). The low Reynolds number DNS results of Kline and Robinson (1989), Robinson (1991), and high Reynolds number particle image velocimetry (PIV) data of Adrian et al. (2000) indicate that these asymmetric hairpins dominate the turbulent boundary layer. Following Adrian et al. (2000), the term "hairpin" will be used in the current study to represent any structures that resemble a horseshoe or hairpin and whether they are symmetric or asymmetric, since these structures appear to be variations of the same flow structure.

The formation of a hairpin vortex can be described as shown in Figure 2.5. When a localized shear layer becomes unstable (for example, in the non-linear stages of the T-S wave growth or in the presence of an inflectional velocity profile created due to an external disturbance), it rolls up as shown in Figure 2.5(a) with a sense of rotation as indicated by the arrow. The vorticity in this shear layer is primarily oriented in the spanwise direction. The form of the roll-up is independent of the amplitude and orientation of an initial disturbance in a wall-bounded shear layer (Smith et al., 1991). The vortex, once deformed by small perturbations in the local flow field, is then stretched 
in the streamwise direction by the mean shear of the background flow elongating it to form quasi-streamwise counter-rotating vortices which are called as "legs" of the vortex, with the downstream ends of the legs connected to the spanwise vortex as illustrated in Figure 2.5(b). The sense of rotation in the legs is also indicated in Figure 2.5(b). The velocity field induced by the deformed vortex results in the "head region" of the vortex to move away from the wall. The higher local velocities prevailing farther away from the wall result in stretching of the vortical structure, providing it the characteristic hairpin shape. Additional modes of instability result in the development of similar vortical patterns on either side of the hairpin vortex as shown in Figure 2.5(c). Zhou et al. (1999) observe that once the hairpin vortex forms and moves away from the surface due to mutual- and self-induction, the legs tend to spread further apart but the spanwise spacing of the heads of neighbouring hairpin vortices has been observed to be about 100 wall units in a turbulent boundary layer. The spanwise spacing of the legs is dependent on the strength of the near-wall mean shear of the background flow with smaller spacing obtained for flows with higher near-wall mean shear (Hon and Walker, 1987).

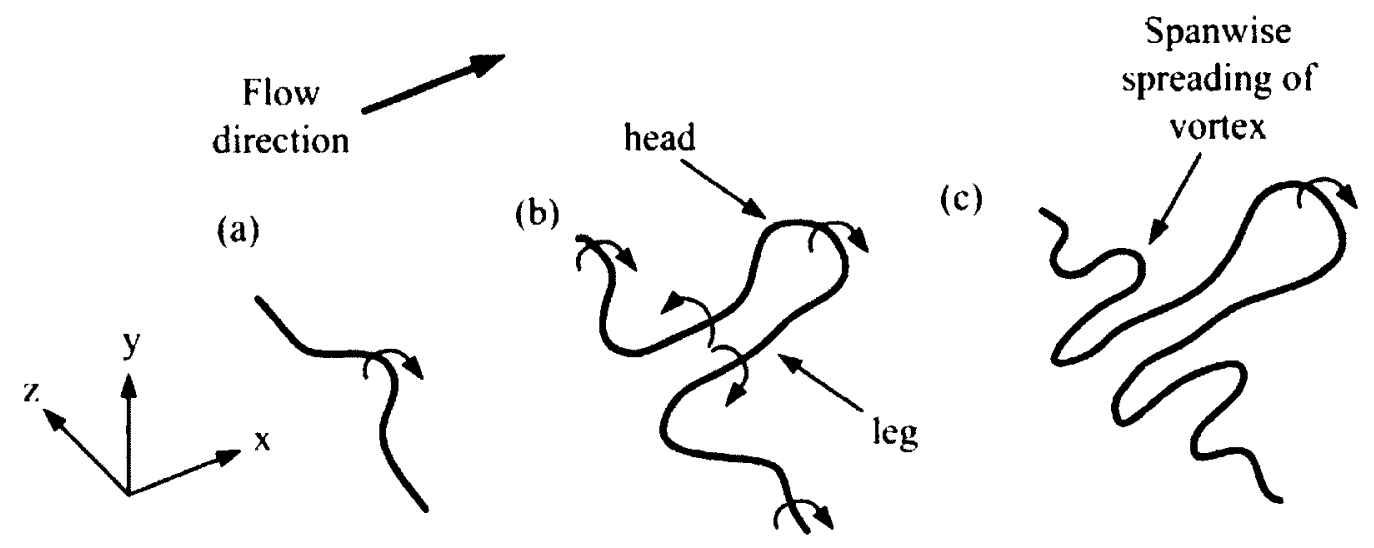

Figure 2.5: Evolution of a symmetric hairpin vortex in a shear flow: (a) initial distortion, (b) development of vortex legs and head, (c) evolution of subsidiary vortices and penetration toward the surface (adapted from Smith et al., 1991). 
The sense of rotation in the head and legs of a hairpin indicates the pattern of fluid exchange near the wall that is induced by the hairpin vortex, which can be represented as shown in Figure 2.6. The lower-speed fluid closer to the wall is induced away from the wall into the higher-speed region between the head and the legs of the hairpin vortex; this is known as an ejection event. On the outboard side of the head and legs of the hairpin vortex, higher-speed fluid is induced towards the low-speed region, and this is known as a sweep event (Adrian et al., 2000). This pattern of fluid exchange can be graphically represented using the streamwise and wall-normal components of velocity fluctuations $\left(u^{\prime}\right.$ and $\left.v^{\prime}\right)$ as shown in Figure 2.7. Accordingly, ejection and sweep events are also referred to as the Q2 and Q4 events, respectively. The stagnation point shown in Figure 2.6 is formed where the Q2 and Q4 events cancel each other out.

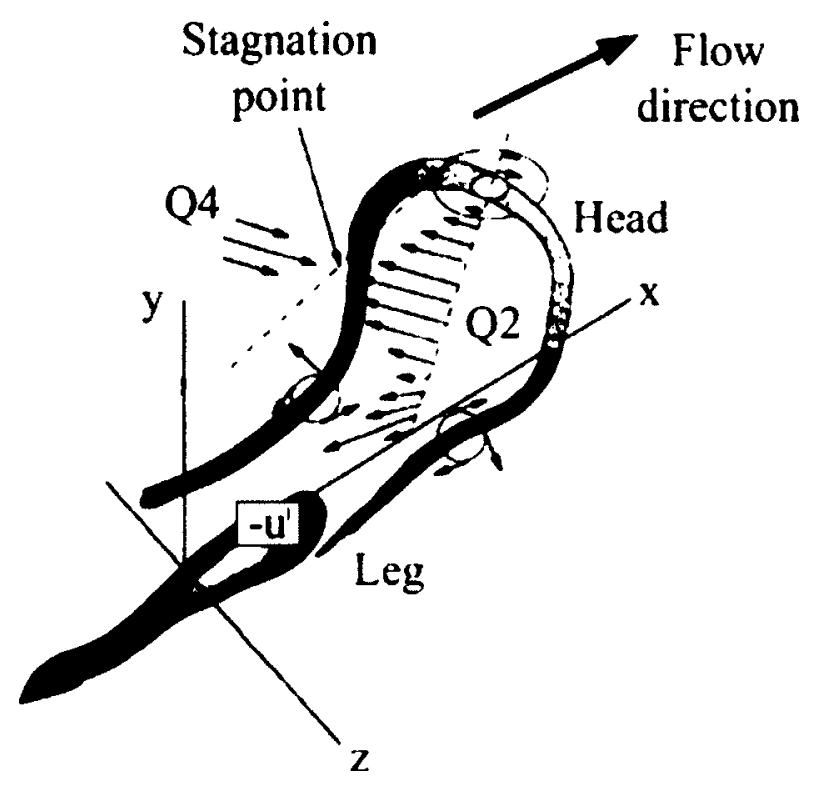

Figure 2.6: Schematic of a hairpin vortex attached to the wall (reproduced from Adrian et al., 2000). 


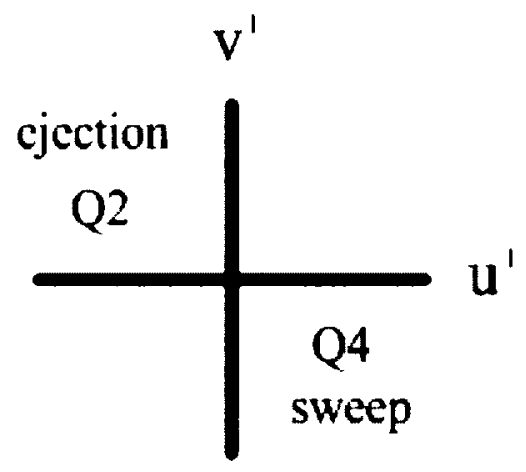

Figure 2.7: Quadrants of the $u^{\prime} v^{\prime}$ plane indicating sweep and ejection events (adapted from Robinson, 1991).

The induced wall-normal motions (Q2 and $\mathrm{Q} 4$ events) are believed to be responsible for the near-wall occurrence of alternating high- and low-speed fluid regions known as streaks (Hon and Walker, 1987, Robinson, 1990, Zhou et al., 1999). Figure 2.6 shows a low-speed streak by the contour of the negative component of the streamwise fluctuating velocity $\left(-u^{\prime}\right)$. Smith et al. (1991) conceptualize that two pairs of streaks (one pair consists of a low-speed and a high-speed streak) would be created by a symmetric hairpin vortex whose legs are spaced fairly apart, with each pair of streak associated with one leg of the symmetric vortex. Figure 2.8 (a) shows only the low-speed streaks due to each leg of the hairpin vortex for clarity. If the legs of the symmetric vortex are closely spaced (Figure 2.8(b)), the two low-speed streaks due to each leg of the vortex would merge and appear to indicate a single low-speed streak. However, if the vortex is asymmetric, then the dominant leg of the vortex would create a single low-speed streak (Figure 2.8(c)). At lower Reynolds numbers, the streak patterns depicted in Figure 2.8 (a) and (b) are commonly observed (Smith et al., 1991) but at higher Reynolds numbers, the 
streak pattern depicted in Figure 2.8(c) is more commonly observed due to the dominance of asymmetric hairpin vortices.

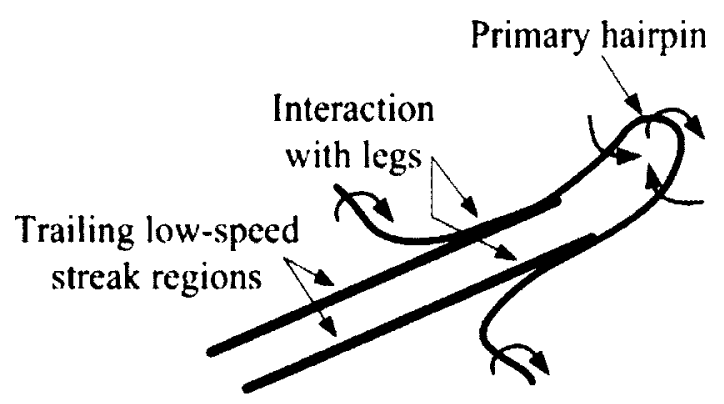

(a)

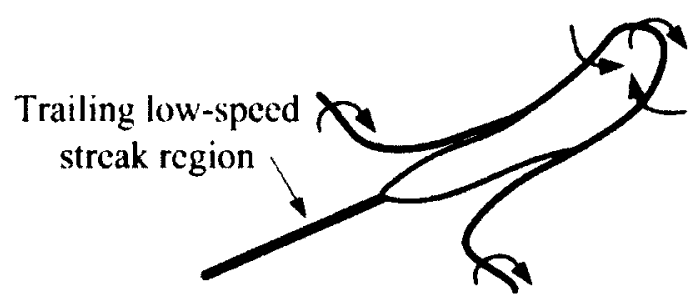

(b)

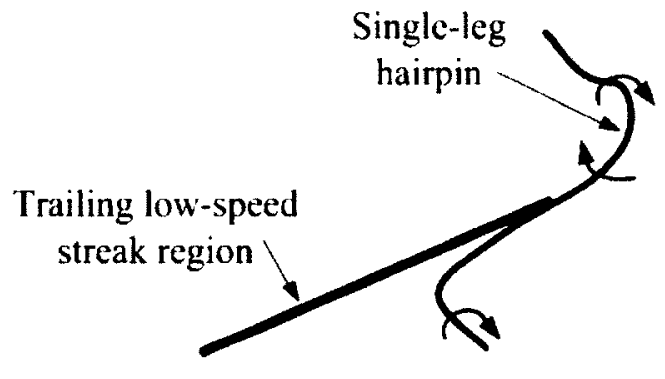

(c)

Figure 2.8: Hairpin vortices inducing low-speed streaks: (a) symmetric case with wide separation of legs, (b) symmetric case with narrow leg separation, (c) asymmetric case (reproduced from Smith et al., 1991).

Chernyshenko and Baig (2005) state that streaks are universal in wall-bounded turbulent flows as they are observed in almost all experimental and DNS studies of nearwall turbulent flows. The streak dimensions in the streamwise and spanwise directions in turbulent boundary layers are $\Delta x^{+} \approx 1000$ and $\Delta z^{+} \approx 100$, respectively. The superscript "+" denotes normalization by the wall variables, friction velocity $\left(u_{\tau}\right)$ and kinematic viscosity (v) which are described in Appendix A.1. In a channel flow simulation by Kim (1983), the average streamwise length of a low-speed streak associated with a single hairpin vortex is observed to be $\Delta x^{+} \approx 200$ to 300 which is consistent with the channel flow 
simulation of Zhou et al. (1999). A process commonly observed in turbulent boundary layers is "bursting" associated with production of turbulence kinetic energy and wallnormal momentum exchanges (ejection and sweep events) in the boundary layer (Kline et al., 1967). In experiments, multiple ejection events have been detected during a single burst event, which is not possible with a hairpin vortex in isolation as that would cause only a single ejection event. Observations of long low-speed streaks and multiple ejection events during a single burst event suggest the presence of a sequence of hairpin vortices rather than a single hairpin vortex. The DNS studies of Zhou et al. (1999) and the PIV studies of Adrian et al. (2000) reveal that the hairpin vortices do occur in groups consisting of streamwise aligned hairpin vortices, with the size of individual hairpins increasing in the downstream direction. Such organization of hairpin vortices is referred to as wave packets of hairpin vortices. A wave packet in the DNS of a channel flow by Zhou et al. (1999) is shown in Figure 2.9. The development of the wave packet can be described as follows: an initial hairpin vortex is formed as described earlier due to a rollup of an unstable shear layer which is created due to a disturbance in the boundary layer yielding an inflectional instantaneous velocity profile. In the DNS of Zhou et al. (1999), a three-dimensional (3-D) vortex that is extracted from a two-point spatial correlation of the velocity field by the method of linear stochastic estimation applied to a turbulent channel flow data of Kim et al. (1987), is imposed in a background mean velocity profile similar to that of a turbulent flow at $R e_{\tau}=180\left(R e_{\tau}=u_{\tau} \delta / v\right.$, where $u_{\tau}$ is the friction velocity, $\delta$ is the boundary layer thickness and $v$ is the kinematic viscosity). The extracted structure represents a quasi-streamwise vortex with an associated Q2 event, leading to a local inflection point in the velocity field right above the initial structure. The resultant 
unstable shear layer rolls up into the primary hairpin vortex (PHV) as shown in Figure 2.9. The secondary hairpin vortex (SHV) is then created from the PHV by an interaction of the low-speed fluid induced upwards between the legs of the PHV and the high-speed fluid above the legs, leading to a local unstable shear layer (Adrian, 2007). This shear layer rolls up and then connects to the legs of the PHV to form the SHV. The tertiary hairpin vortex (THV) forms from the SHV in a similar manner. Zhou et al. (1999) also observe the formation of a hairpin vortex downstream of the PHV due to protrusions of the initial structure in the downstream direction from the PHV. These protrusions act in a similar manner as legs of a hairpin vortex to induce an upward flow between them, thus forming an unstable shear layer that rolls up to form the downstream hairpin vortex (DHV) as seen in Figure 2.9. Such downstream hairpin vortices have not been commonly observed in turbulent boundary layers, but Zhou et al. (1999) consider them to be realistic flow structures and not just an effect of their imposed initial 3-D vortex. The authors suggest that there is some evidence of downstream protrusions from the PHV in a flow visualization experiment of Haidari and Smith (1994) but the occurrence of downstream hairpin vortices in turbulent boundary layers remains inconclusive.

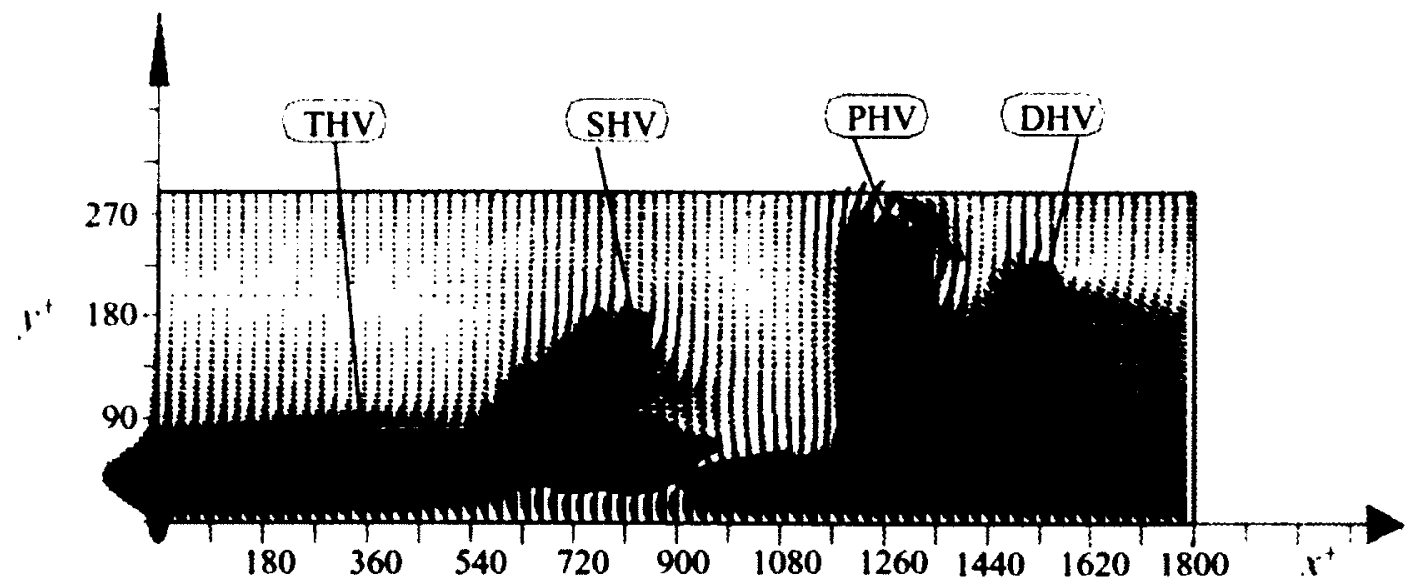

Figure 2.9: Hairpin wave-packet (reproduced from Zhou et al., 1999). 
As the Reynolds number of the flow increases, the separation of length scales of the wave packets increases, with smaller wave packets existing close to the wall and larger wave packets existing farther away from the wall. The simultaneous existence of multiple hairpin vortex packets in the turbulent boundary layer leads to an interaction between these different wave packets creating a complicated pattern of vortices (Adrian et al., 2000). Due to the presence and mutual interaction of multiples of such structures, the detection of a single hairpin vortex or a wave packet is an intricate process. However, some of the characteristic features of a hairpin vortex assist in its detection. For example, a concentrated region of spanwise vorticity may be indicative of the head of the hairpin, a Q2 event may suggest the presence of hairpin legs, and a stagnation point may indicate the destructive combination of the Q2 and Q4 events. In addition, the presence of a lowspeed streak strongly indicates the presence of a hairpin vortex and the combination of these factors is shown in Figure 2.10 which is referred by Adrian et al. (2000) as the hairpin vortex signature. This signature can be of assistance in the detection of hairpin vortices in the results of an experiment or DNS.

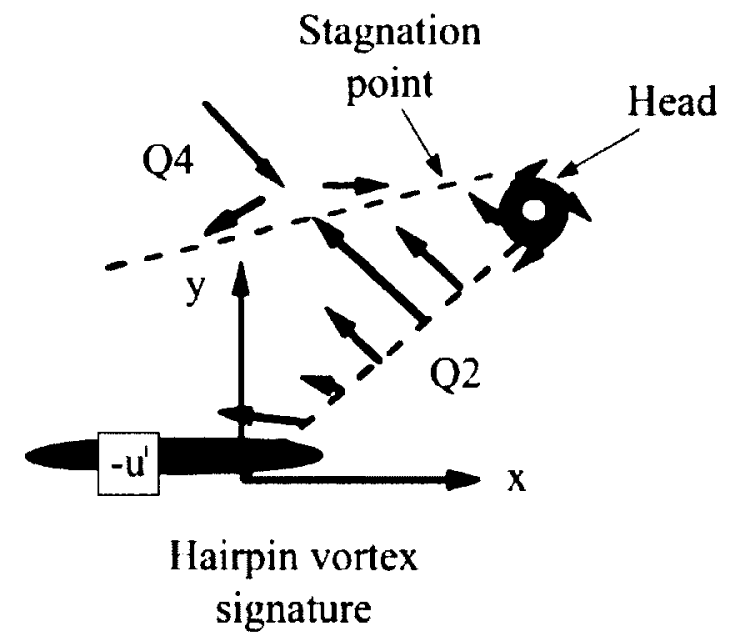

Figure 2.10: Hairpin vortex signature in the streamwise-wall-normal plane (reproduced from Adrian et al., 2000). 
Several researchers initiate the development of hairpin vortices in a "clean" background laminar or turbulent flow using different methods of disturbing the boundary layer and study the further growth and development of the hairpin vortices. In the experiment by Acarlar and Smith (1987b), fluid injection is used to disturb the laminar boundary layer which forms an initial hairpin, and subsequent hairpins are created by this initial hairpin. Singer and Joslin (1994) also trigger an initial hairpin vortex through wallnormal fluid injection in a spatial DNS of a laminar boundary layer and observe the development of multiple hairpin vortices from the initial vortex. Wu and Moin (2009) introduce isotropic patches of turbulence periodically in the freestream of a laminar boundary layer of their spatial DNS and observe the development of hairpin wave packets. A significant outcome of the above-mentioned studies and many others is that irrespective of the study, wave packets of hairpin vortices have been observed in transitional and fully turbulent boundary layers. Since hairpin vortices play a significant role in the mass and energy transport in the boundary layer, it is logical to study turbulent boundary layers in different flow environments in terms of the behaviour and interaction of such structures.

\subsection{Effect of Streamwise Convex Surface Curvature on Turbulent Boundary Layers}

The flow over curved surfaces is experienced in many practical situations such as flow past wing surfaces, bluff bodies and turbomachinery flows. The presence of surface curvature can have a significant effect on the boundary layer as compared to the flow over a flat surface, as streamwise surface curvature gives rise to centrifugal and pressure 
forces on the fluid particles. Due to the presence of these forces, the assumption of negligible variation of the static pressure across two dimensional boundary layers in the wall-normal direction is valid on a flat surface but not on convex surfaces. The turbulence activity within the boundary layer developing on a streamwise convex surface is reduced compared to a flat surface which implies that convex surface curvature exerts a stabilizing influence on the boundary layer (So and Mellor, 1978; Gowda and Narayana, 1980). Such stabilizing influence of streamwise curvature in shear layers developing on convex-shaped walls is well established (Gillis and Johnston, 1983), and is the result of the radial equilibrium that exists between wall-normal pressure gradients and the centrifugal force acting on the fluid particles due to their centripetal acceleration. The centrifugal force and thus the radial pressure gradient are proportional to the square of the streamwise velocity component, and therefore have a stronger magnitude with increasing wall-normal distance.

In order to quantify the magnitude of curvature effects on a boundary layer, Liepmann (1943) adopts the dimensionless parameter, $\delta / R$ where $\delta$ is the boundary layer thickness and $R$ is the radius of curvature of the test surface, due to the following reason: assuming two-dimensional steady flow with negligible values of wall-normal velocity reduces the wall-normal momentum equation to the following equation:

$$
\frac{\rho u^{2}}{r}=\frac{\partial p}{\partial r}
$$

where, $\rho$ is the flow density, $u$ is the streamwise velocity component, $r$ is the local radial coordinate and $p$ is the static pressure. This equation can be rearranged in the form presented below to signify radial pressure variation across the boundary layer: 


$$
\frac{\Delta p}{\rho u^{2}} \approx \frac{\Delta r}{R}=\frac{\delta}{R}
$$

where the radius of curvature of the test surface $(R)$ can be considered constant due to a negligible variation in the radius value across the boundary layer. Thus, the parameter $(\delta / R)$ can be suitably used to indicate the effect of curvature as it provides an estimate of the balance between the radial pressure gradient and the centrifugal force in the boundary layer on a curved surface. Several experiments have shown that based on the stabilizing effect of streamwise convex curvature, surface curvatures corresponding to $\delta / R<0.02$ are identified as mild curvatures and those corresponding to $\delta / R>0.05$ are identified as strong curvatures (So and Mellor, 1973; Meroney and Bradshaw, 1975; Ramaprian and Shivaprasad, 1978; Gillis and Johnston, 1983).

The effects of streamwise curvature on turbulent flows were shown to be analogous to buoyancy effects by Bradshaw (1969) which resulted in the MoninOboukhov formula for the apparent mixing length with small buoyancy or curvature effects:

$$
\frac{l}{l_{0}}=1-\beta R i
$$

where $l$ and $l_{0}$ are the mixing lengths with and without buoyancy (or curvature) respectively, $\beta$ is a constant with a value between 7 and 10 for convex curvature, and $R i$ is called the Richardson number defined for curved flows as:

$$
\begin{gathered}
R i=2 S(1+S) \\
S=\frac{u / R}{\partial u / \partial r}
\end{gathered}
$$

where $\partial u / \partial r$ is the mean shear and $R i \approx 2 S$ for large values of the radius or low curvatures. Bradshaw (1969) used the above relations to find that even for a mildly- 
curved convex surface with $\delta / R=0.0125$, the skin friction coefficient $\left(C_{f f}\right)$ reduces to 0.89 times the value for a flat surface at similar momentum-thickness Reynolds numbers $\left(R e_{\theta}\right)$. This observation prompted subsequent researchers to perform detailed investigations of convex surface flows.

\subsubsection{Typical Experimental Arrangement in the Study of Convex Surface Curvature Effects on Turbulent Boundary Layers}

The experiments performed to study the effects of curvature on boundary layers normally consist of high aspect ratio ducts to avoid secondary flows and adequate height to have an inviscid core region so that the boundary layers on the concave and convex sides do not affect each other. A typical experimental arrangement is shown below:

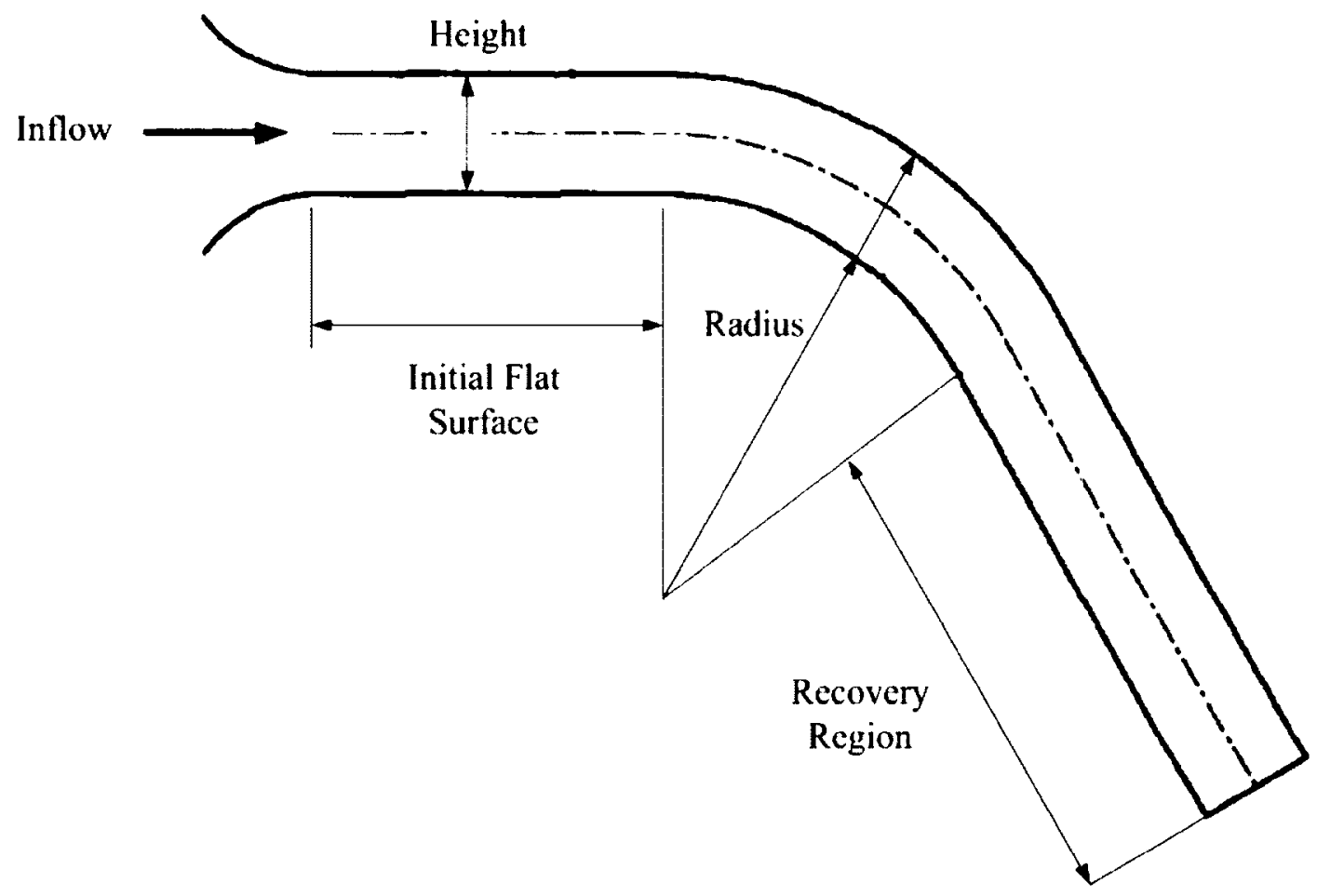

Figure 2.11: Experimental arrangement (adapted from Patel and Sotiropoulos, 1997). 
The initial flat surface is provided for boundary layer development where trip wires are extensively used (Gibson et al. (1984) amongst others) such that the boundary layer is fully turbulent before the beginning of the curved section. The recovery region, which is also a flat surface, is used by some researchers to study the recovery of the turbulent boundary layer from the effects of curvature and also to ensure the outlet is a fair distance from the curved section to diminish any effects on the curved-surface boundary layer. A contoured ceiling (the concave side is contoured to study the development of the boundary layer on the convex side) is also used by some researchers (Gillis and Johnston, 1983; Chiwanga and Ramaprian, 1993) to maintain a nearly zero longitudinal pressure gradient across the curved section. Experiments with convex surface curvatures cited in this thesis are presented in Table 2.2 with the relevant experimental parameters. 
Table 2.2: Experiments conducted using convex surface curvatures.

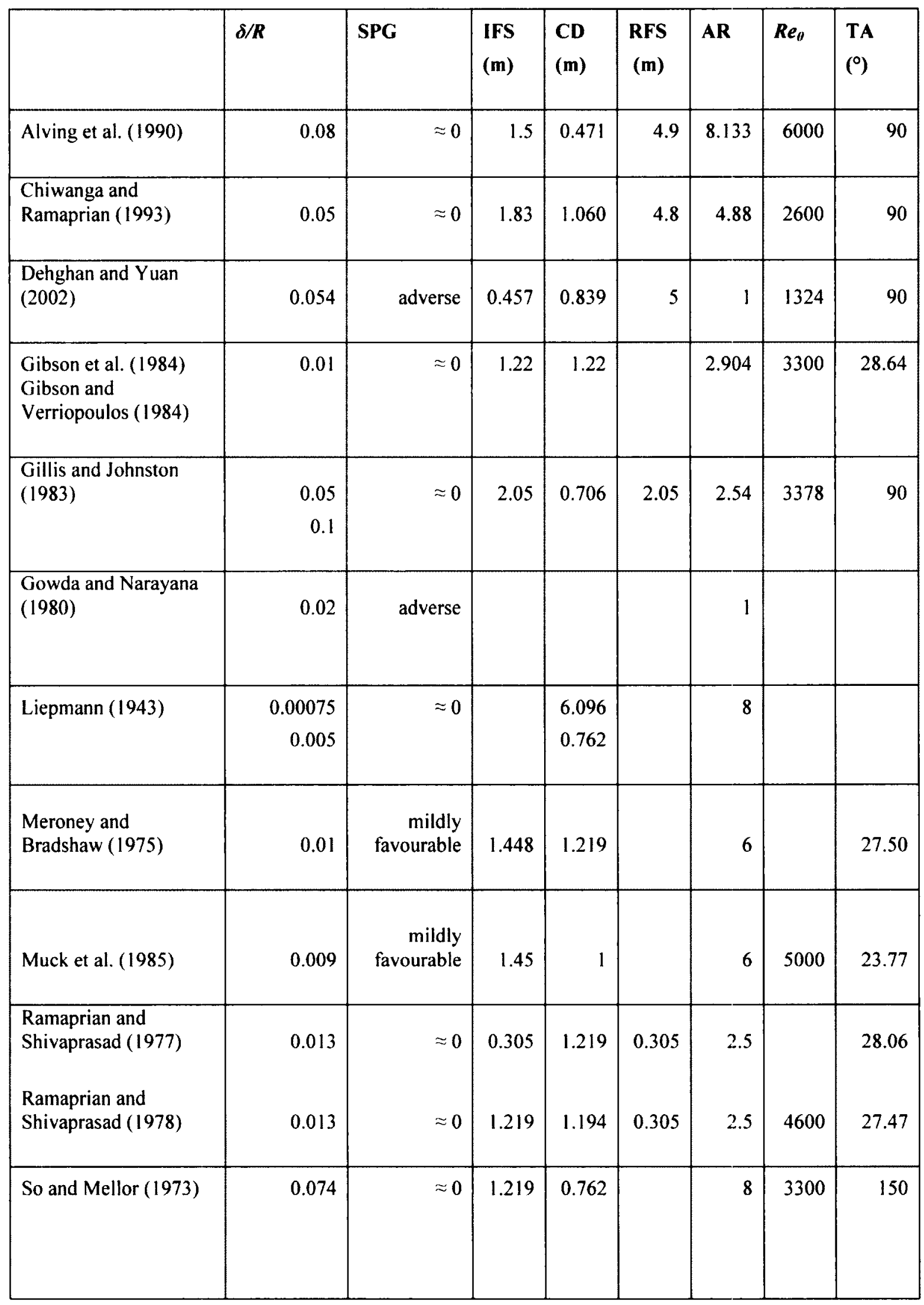




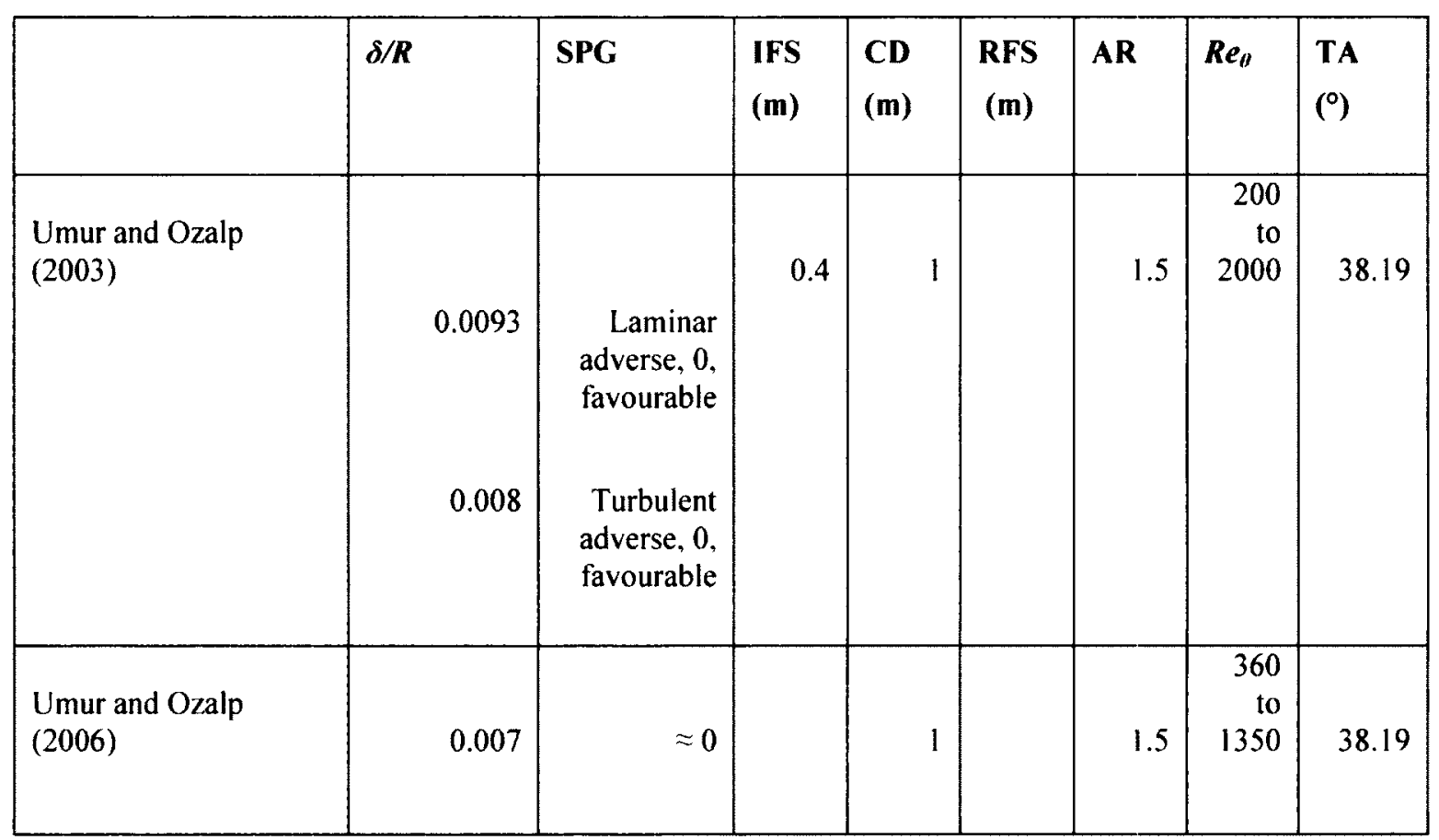

SPG - Streamwise pressure gradient, IFS - Initial flat surface, CD - Curved duct, RFS Recovery flat surface, AR - Aspect ratio (width/height of duct), TA - Turn angle.

\subsubsection{Effect of Streamwise Convex Surface Curvature on the Mean Turbulent Velocity Profile}

The measurements of the mean velocity profile in a turbulent boundary layer by Meroney and Bradshaw (1975) indicate that the boundary layer is thinner on the convex surface in comparison to a flat surface at the same $\operatorname{Re}_{x}\left(\operatorname{Re}_{x}=u_{x} x / v\right.$, where $u_{x}$ is the freestream velocity of the flow, $x$ is the streamwise distance along the test surface and $v$ is the kinematic viscosity), and Gibson et al. (1984) observe that the velocity profiles on the convex surface are less full than that of the flat surface. Several experiments with almost negligible streamwise pressure gradients on flows over convex surfaces (So and Mellor, 1973 and Gillis and Johnston, 1983 amongst others) have shown that the mean velocity profiles on convex surfaces lie above the log-law region applicable to flat surface cases 
with the difference increasing with stronger curvatures. The mean velocity profiles of Ramaprian and Shivaprasad (1977) are shown in Figure 2.12 which indicates that in the inner region of the boundary layer, the velocity profiles for the convex surface are in accordance with the law of the wall for the flat surface but in the outer region, substantial deviation occurs. The experiments by Muck et al. (1985) with a mildly favourable streamwise pressure gradient and Gowda and Narayana (1980) with an adverse pressure gradient show a similar deviation of the mean velocity profiles. Attempts have been made to modify the existing log-law relation to correspond with the observation of the mean velocity profiles on curved surfaces. So (1975) derived a law of the wall for curved flows by equating the production rate and the dissipation rate of turbulence kinetic energy and assuming a prescribed variation of the turbulence shear stress while Patel and Sotiropoulos (1997) assume constant turbulence shear stress and neglect the extra term due to curvature in the equation for the production of turbulence kinetic energy. The relations developed by these authors are only applicable to flows bounded by a convex surface and are therefore not widely used when compared to the more general flat surface law of the wall. The log-law is an important relation for determining parameters such as the skin friction coefficient and for developing turbulence models (Patel and Sotiropoulos, 1997) but the use of the flat surface log-law for curved flows is bound to introduce errors due to the deviation of the convex surface velocity profiles from a flat surface that is apparent from several experiments. Thus, the existing log-law correlations need to be modified to accurately capture the velocity profiles over curved surfaces by an understanding of the flow physics over curved surfaces through detailed experiments and DNS studies. 


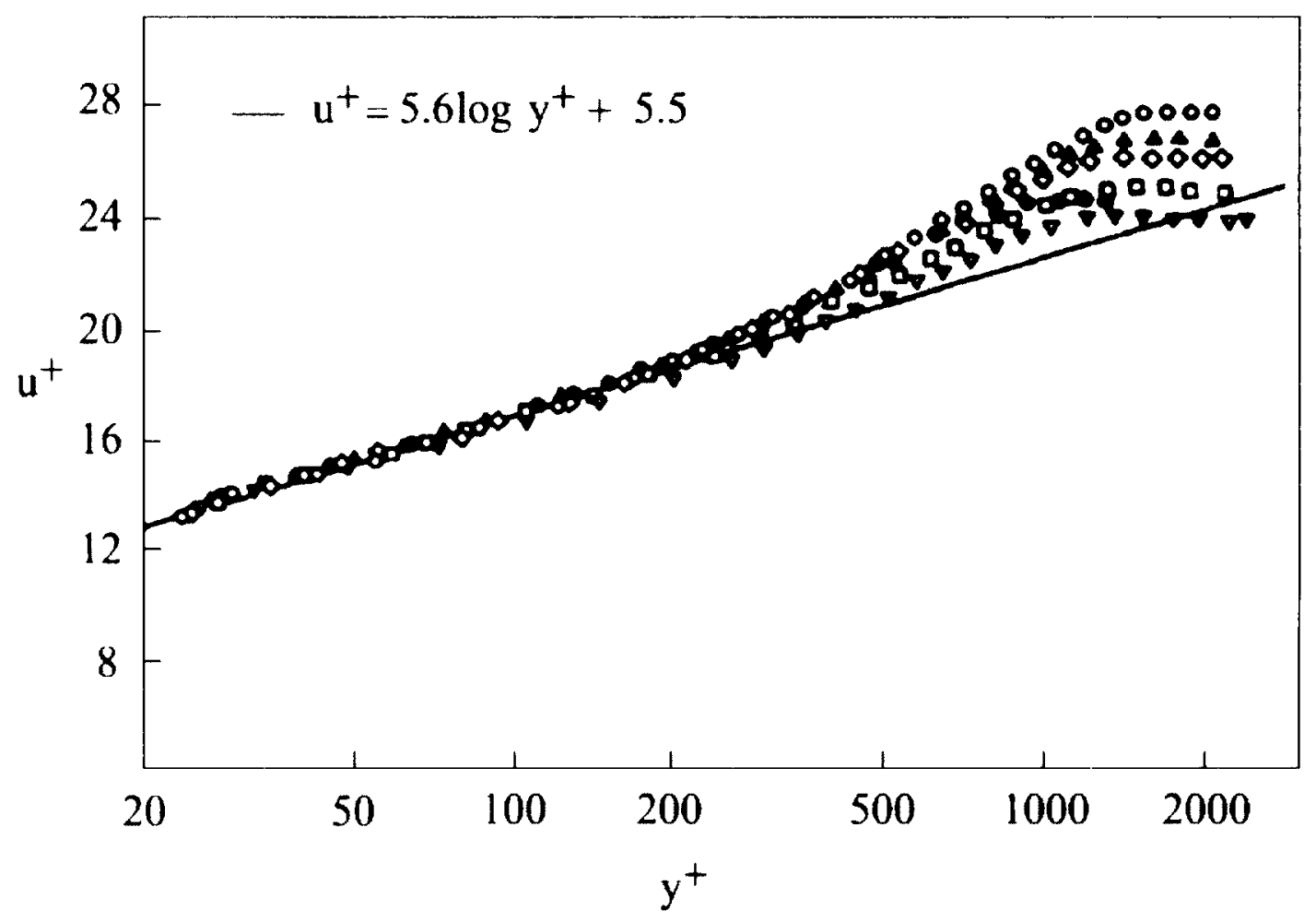

Figure 2.12: Mean velocity profile on a convex surface (adapted from Ramaprian and Shivaprasad, 1977). The symbols represent different measuring stations along the curved surface with the difference from the flat-surface law of the wall increasing with distance along the curved surface.

\subsubsection{Effect of Streamwise Convex Surface Curvature on the Turbulent Boundary}

Layer

This section describes the effect of mildly-curved and strongly-curved convex surfaces on turbulent boundary layers. Corresponding information on the effect of convex surface curvature on transitioning flows is given in Appendix A.2. 


\subsubsection{Effect of Mildly-Curved Convex Surfaces on Turbulent Boundary Layers}

Experiments with convex surface curvature employ an initial flat surface region to allow for boundary layer development to a fully turbulent state. As the flat-surface boundary layer develops to a fully turbulent state, the shape factor decreases and almost levels off indicating the end of the transition period ( $\mathrm{Wu}$ and Moin, 2009). The introduction of streamwise convex surface curvature following the initial flat surface region causes an increase in the shape factor by almost $6 \%$ to $8 \%$ as compared to the value on the flat surface if convex surface curvature had not been introduced (Meroney and Bradshaw, 1975; Ramaprian and Shivaprasad, 1977; Gibson et al., 1984). These authors also observe the value of the skin friction coefficient on the convex surface to be almost $88 \%$ of that on the flat surface. However, there is some disagreement with regards to the growth rate of the momentum thickness Reynolds number in the flat surface and convex surface boundary layers. Meroney and Bradshaw (1975) observe a reduced momentum thickness growth rate while Ozalp and Umur (2003) observe a higher growth rate on the convex surface; Ramaprian and Shivaprasad (1977) find that the growth rate of the momentum-thickness Reynolds number decreases initially but increases later on the convex surface in comparison to the flat surface. Ramaprian and Shivaprasad (1977) attribute the initial decrease in the momentum thickness growth rate to a possible result of the sudden introduction of flow to curvature from initial flat surface conditions and the later increase in the momentum thickness growth rate to the flow being subjected to sustained curvature. However, the less full velocity profile and an increasing shape factor of the boundary layer on the convex surface is suggestive of a reduced growth rate of the momentum-thickness Reynolds number in comparison to the flat surface boundary layer. 
A small aspect ratio duct with secondary flows and the influence of a slightly favourable streamwise pressure gradient in the experimental investigations of Ramaprian and Shivaprasad (1977) may have an effect on the convex surface boundary layer, although the authors persist that secondary flows in their test section are insignificant above and below the plane of measurement and the boundary layer is dominated by curvature effects.

The values of the Reynolds normal stresses $\left(\overline{u^{\prime 2}}, \overline{v^{\prime 2}}, \overline{w^{\prime 2}}\right)$ and the corresponding values of the root-mean-square of the turbulence velocity fluctuations $\left(u_{r m s}^{\prime}, v_{r m s}^{\prime}\right.$, $w^{\prime}{ }_{r m s}$ ) within the boundary layer on the convex surface are reduced to about $80 \%$ of that on the flat surface. Convex surface curvature also reduces the values of the Reynolds shear stress $\left(\overline{u^{\prime} v^{\prime}}\right)$, with most of the suppression occurring in the outer part of the boundary layer (Meroney and Bradshaw, 1975; Gibson et al., 1984; Muck et al., 1985; Moser and Moin, 1987). Gibson et al. (1984) attribute the lower Reynolds shear stress values to the effects of extra strain due to curvature $(u / R)$ and the low values of the wallnormal Reynolds stress $\left(\overline{v^{\prime 2}}\right)$ which appear in the transport equation for the Reynolds shear stress. This effect is most pronounced away from the wall due to the increase in the strain-rate ratio. The triple velocity products $\left(\overline{u^{\prime 2} v^{\prime}}, \overline{v^{\prime 3}}, \overline{u^{\prime} v^{\prime 2}}\right)$ represent the turbulence diffusion fluxes for the Reynolds normal stress (first two terms) and the Reynolds shear stress, and these values are substantially reduced in the boundary layer on the convex surface with the most effect seen on $\left(\overline{v^{\prime 3}}\right)$ as compared to the flat surface indicating that the wall-normal momentum transport is significantly reduced due to the stabilizing effects of streamwise convex surface curvature (Gibson et al., 1984). 
The suppression of the wall-normal velocity fluctuations in the boundary layer on a convex surface inhibits the outward diffusion of turbulence kinetic energy from the near-wall region to the outer part of the boundary layer. The extent of the near-wall region that supplies energy to the outer region of the boundary layer is also diminished on the convex surface in comparison to the flat surface, which confines the effect of the wall to a smaller region close to the wall (Ramaprian and Shivaprasad, 1978). The effects of curvature increase away from the surface where the boundary layer is developing but close to the boundary layer surface, the wall-effect dominates which suggests why the convex-surface velocity profiles deviate from the flat-surface log-law equation away from the near-wall region.

Ramaprian and Shivaprasad (1982) calculate the integral time scales from the auto-correlation functions of the streamwise and wall-normal velocity fluctuations and find them to be smaller for the convex surface as compared to the flat surface. Since the integral time scales give a measure of the duration for which the fluctuations last, thereby giving a measure of the lifetime of the eddies, it is indicative that the average lifetime of the eddies is longer on the flat-surface than the convex-surface boundary layer. Ramaprian and Shivaprasad (1982) also calculate the spectra of the Reynolds shear stress revealing that half of the contribution to the Reynolds shear stress on the convex surface is obtained from eddies smaller than a quarter of the boundary layer thickness whereas similar eddies contribute much less to the Reynolds shear stress on the flat surface. This implies that the larger eddies which contribute to the remaining Reynolds shear stress are attenuated over the convex surface, indicating the increased sensitivity of the larger structures to convex surface curvature. 


\subsubsection{Effects of Strongly-Curved Convex Surfaces on Turbulent Boundary Layers}

For strongly curved convex surfaces, the Reynolds shear stress in the outer part of the boundary layer $(y / \delta>4)$ is almost completely eliminated and the Reynolds normal stresses also fall to zero before the edge of the boundary layer is reached in comparison to the flat surface boundary layer (So and Mellor, 1973; Gillis and Johnston, 1983; Dehghan and Yuan, 2002). In order to eliminate any effect other than that of curvature, So and Mellor (1973) maintained a near-zero streamwise pressure gradient by adjusting the opposite concave wall and employed a large aspect ratio duct to eliminate secondary flow effects within their measurement zone. They also installed small end-wall jets to introduce momentum that would compensate for the momentum deficit at the end-walls. Gillis and Johnston (1983) also maintained a near-zero streamwise pressure gradient by adjusting the contour of the opposite concave wall. Such effects on the Reynolds stresses due to strongly-curved streamwise convex surfaces led Gillis and Johnston (1983) to suggest a two-layer structure in the boundary layer over the convex surface with a nearwall region exhibiting almost similar characteristics to a flat surface and the outer region away from the wall where the increased influence of the stabilizing effect of convex curvature eliminates the stress generating mechanisms. This implies that the larger scale structures either shrink in size or are destroyed closer to the edge of the boundary layer. In the recovery flat surface region following the convex surface region, the turbulence does not immediately return to standard flat surface conditions even more than $20 \delta$ from the end of the curved section (Gillis and Johnston, 1983; Alving et al., 1990). This is a likely consequence of the destruction of the larger scale structures near the edge of the 
boundary layer on the convex surface; since the larger scale structures are also the oldest that carry information about the upstream initial flat surface flow, some of the flow history is lost (Gillis and Johnston, 1983). The boundary layer recovers slowly in the recovery region with the small-scale structures growing in the same manner as they would in a developing boundary layer on a flat surface. This concept by Gillis and Johnston (1983) is supported by the flow visualization experiment of Chiwanga and Ramaprian (1993) who conducted tests in a specially-designed wind tunnel with a nearlyzero streamwise pressure gradient maintained by allowing the adjustment and bleeding of fluid through the opposite concave wall. The flow visualization was conducted using a laser light sheet obtained with an argon ion laser and smoke that was introduced into the boundary layer through a slot on the surface. The images using this technique show an absence of large scale structures near the edge of the boundary layer on the convexsurface as compared to the preceding flat-surface boundary layer, and the smaller structures on the convex surface are confined to the inner region of the boundary layer. The boundary layer did not recover immediately following the removal of curvature and even at a distance of about $33 \delta$ after the removal of curvature, only a few large scale structures seem to appear in the recovery flat surface region.

\subsubsection{Effect of Streamwise Convex Surface Curvature on Convection Heat}

\section{Transfer}

The mean temperature profiles in the boundary layer on a convex surface are less full than the corresponding profiles on the flat surface similar to the behaviour of the mean velocity profiles. The value of the Stanton number $\left(S t=h / \rho u_{\infty} C_{p}\right.$, where $h$ is the 
convection heat transfer coefficient, $\rho$ is the flow density, $u_{\infty}$ is the freestream velocity, $C_{p}$ is the specific heat at constant pressure) is slightly more affected by convex surface curvature as it is reduced to $82 \%$ compared to $C_{f}$ reducing to $88 \%$ of that on the flat surface (Gibson and Verriopoulos, 1984; Ozalp and Umur, 2003). The values of the longitudinal $\left(\overline{u^{\prime} T^{\prime}}\right)$ and wall-normal $\left(\overline{v^{\prime} T^{\prime}}\right)$ turbulence-driven heat fluxes in the convex surface boundary layer are lower than those in the flat surface boundary layer due to the presence of the $\left(\overline{u^{\prime} v^{\prime}}\right)$ term in the longitudinal and $\left(\overline{v^{\prime 2}}\right)$ term in the wall-normal turbulence heat flux transport equations and also the extra strain term $(u / R)$ in both the transport equations that act to reduce the heat fluxes. The temperature variance $\left(\overline{{T^{\prime 2}}^{2}}\right)$ does not show a significant difference between the convex and the flat surface cases as the equation for this quantity does not contain explicit curved flow generation terms. The turbulence heat diffusion terms, $\overline{v^{\prime 2} T^{\prime}}$ and $\overline{v^{\prime} T^{\prime 2}}$ are significantly reduced showing that heat transport across the boundary layer is diminished on the convex surface as compared to the flat surface (Gibson and Verriopoulos, 1984).

A summary of the effect of streamwise convex surface curvature on a turbulent boundary layer is as follows (based on Patel and Sotiropoulos, 1997): In terms of the law of the wall, the mean velocity profiles of convex surface boundary layers lie well above the flat surface values. There is a slight reduction in the shear stress and total kinetic energy in the inner region of the boundary layer but in the outer region, while the shear stress decreases (almost eliminated for stronger curvatures), the kinetic energy is nearly the same for the convex surface as compared to the flat surface. Convex surface curvature decouples the inner and outer regions of the boundary layer in such a way that the Reynolds shear-stress producing motions are essentially damped in the outer regions with 
the intensity of damping increasing with stronger curvatures. The heat transport across the boundary layer is reduced on convex surfaces as compared to flat surfaces. The response of a turbulent boundary layer (for example, initially developed on a flat surface) to streamwise convex curvature is much faster as compared to the recovery to flat surface conditions. Muck et al. (1985) conclude that convex surface curvature quantitatively affects the existing turbulence structure but new types of eddy structure may not appear. However, the stabilizing effects of streamwise convex surface curvature suggest that there are changes in the development of coherent flow structures within the boundary layer which should be examined in more detail. 


\section{Chapter: Numerical Method and Computational Domain}

Turbulence is a complex process that is three dimensional, unsteady and consists of a varying range of length and time scales. The Navier-Stokes (N-S) equations can effectively describe both laminar and turbulent flows provided the spatial and temporal resolution of the computational model by which these equations are solved numerically is adequate to capture all turbulence scales in the flow. Such simulations that do not involve any modelling related to the effects of turbulence are conventionally referred to as direct numerical simulations (DNS). However, such a grid resolution would substantially increase the cost of the required computational resources and the time taken to solve the $\mathrm{N}-\mathrm{S}$ equations. The rapid increase in power and affordability of computers has enabled such turbulent flow computations to be performed for relatively low Reynolds number flows. The restriction of DNS to low Reynolds numbers stems from the rapid increase in the spectrum of turbulence scales with increasing Reynolds numbers (Fasel, 2006). An adequate number of nodes $(N)$ required in a grid to accurately capture all turbulence

scales increases with the Reynolds number $(R e)$ according to $N \sim(R e)^{9 / 4}$ (Moin and Mahesh, 1998), thus limiting the applicability of DNS to low Re flows.

This chapter documents the simulations performed in this research using DNS, the governing equations and the methods used to solve the flow, and the grid generated to perform the numerical solution. 


\subsection{Governing Equations}

The simulations in this research have been performed using ANSYS CFX $®$ (Version 12.0), which is a commercial computational fluid dynamics software, using the compressible and time-dependent continuity, momentum and energy equations in their conservation form given below in Eqns. 3.1, 3.2 and 3.3 respectively, in their tensor notation.

$$
\begin{gathered}
\frac{\partial \rho}{\partial t}+\frac{\partial}{\partial x_{j}}\left(\rho u_{j}\right)=0 \\
\frac{\partial}{\partial t}\left(\rho u_{i}\right)+\frac{\partial}{\partial x_{j}}\left(\rho u_{j} u_{i}\right)=-\frac{\partial p}{\partial x_{i}}+\frac{\partial \tau_{i j}}{\partial x_{j}} \\
\frac{\partial}{\partial t}\left(\rho h_{0}\right)-\frac{\partial p}{\partial t}+\frac{\partial}{\partial x_{j}}\left(\rho u_{j} h_{0}\right)=\frac{\partial}{\partial x_{j}}\left(k \frac{\partial T}{\partial x_{j}}\right)+\frac{\partial}{\partial x_{j}}\left(u_{i} \tau_{i j}\right) \\
\tau_{i j}=\mu\left(\frac{\partial u_{i}}{\partial x_{j}}+\frac{\partial u_{j}}{\partial x_{i}}\right)
\end{gathered}
$$

where,

$\rho:$ Density $\left(\mathrm{kg} / \mathrm{m}^{3}\right)$

$u_{j}: \quad$ Velocity component $(\mathrm{m} / \mathrm{s})$

$p \quad: \quad$ Pressure $(\mathrm{Pa})$

$\tau_{i j}: \quad$ Stress tensor $(\mathrm{Pa})$

$h_{0}: \quad$ Total enthalpy $(\mathrm{J} / \mathrm{kg})$

$k \quad: \quad$ Thermal conductivity $(\mathrm{W} / \mathrm{m} \mathrm{K})$

$\mu \quad: \quad$ Dynamic viscosity $(\mathrm{kg} / \mathrm{m} \mathrm{s})$

The fluids used in the simulations are assumed to be Newtonian, allowing the stress tensor to be expressed as in Eqn. 3.4. The equations presented above are coupled with the equations of state for the specific working fluid to form a closed system. In this research, 
the working fluids used are subcritical air and supercritical water. Air is approximated as an ideal gas while the International Association for the Properties of Water and Steam (IAPWS) database is used to generate the equation of state for water (IAPWS, 2008; IAPWS, 2009). The IAPWS formulations are utilized by the ANSYS CFX® solver by means of look-up tables specified through pressure and temperature inputs. To prevent extrapolation of property data, the pressure and temperature ranges used for the current study correspond to over four times the expected fluctuation in these properties estimated from similar numerical and experimental studies. The look-up tables are generated with pressure and temperature increments of $0.08 \mathrm{MPa}$ and $0.2 \mathrm{~K}$, respectively. These increment sizes and linear interpolation between the values included in the tables provide values for all relevant thermodynamic properties with an accuracy that is equivalent to the uncertainty associated with the IAPWS equations of state.

\subsection{Discretization}

ANSYS CFX ${ }^{\circledR}$ uses an element-based finite volume method to solve the governing equations, which are integrated over a control volume by converting the volume integrals into surface integrals through Gauss' Divergence Theorem. Eqns. 3.1, 3.2 and 3.3 become:

$$
\begin{gathered}
\frac{\partial}{\partial t} \int_{V} \rho d V+\int_{S} \rho u_{j} d n_{j}=0 \\
\frac{\partial}{\partial t} \int_{V} \rho u_{i} d V+\int_{S} \rho u_{j} u_{i} d n_{j}=-\int_{S} p d n_{j}+\int_{S} \tau_{i j} d n_{j}
\end{gathered}
$$




$$
\frac{\partial}{\partial t} \int_{V} \rho h_{0} d V-\frac{\partial}{\partial t} \int_{V} p d V+\int_{S} \rho u_{j} h_{0} d n_{j}=\int_{S} k\left(\frac{\partial T}{\partial x_{j}}\right) d n_{j}+\int_{S} u_{i} \tau_{i j} d n_{j}
$$

In these equations, $V$ denotes the volume and $S$ denotes the surface regions of integration, and $d n_{j}$ is the differential component of the outward normal surface vector with respect to the surfaces. A control volume is created around the mesh nodes as shown in Figure 3.1. While the present study makes use of three-dimensional elements and volumes, the schematic illustrates a two-dimensional configuration for simplicity.

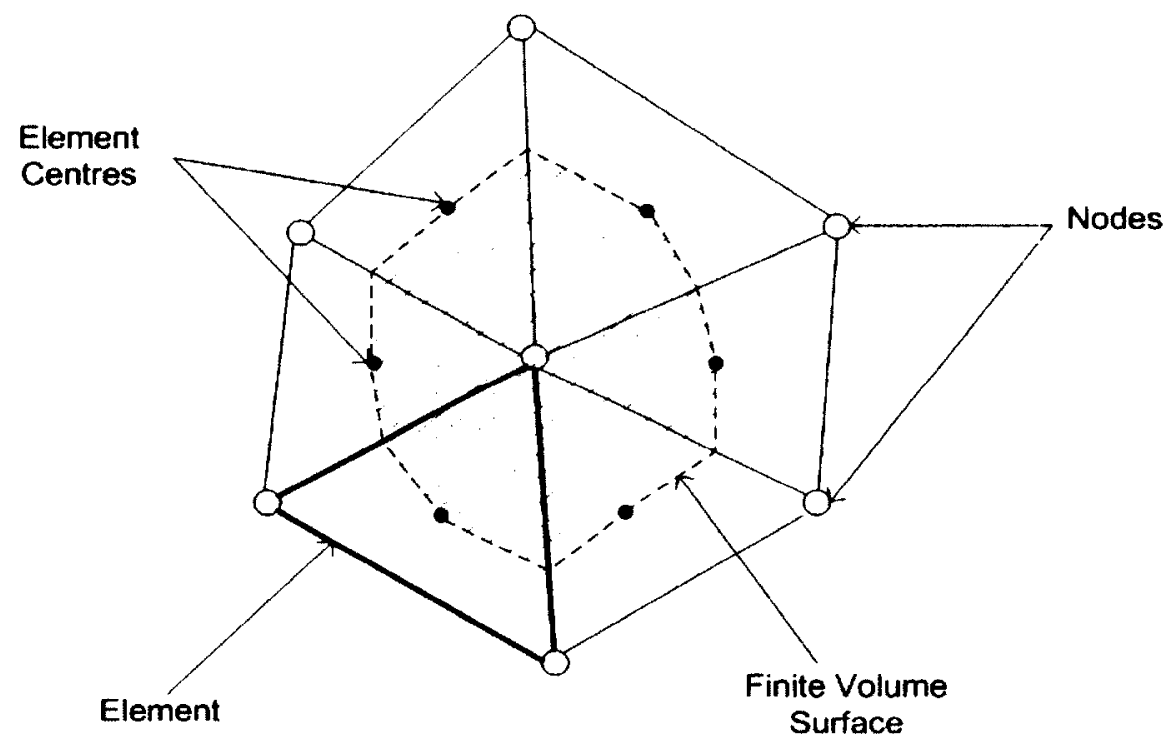

Figure 3.1: Finite volume representation (adapted from ANSYS, 2009)

Once the control volume is set up, a system of linearized algebraic equations are created by discretization of the surface and volume integrals using elements from the control volume as the one shown in Figure 3.2. 


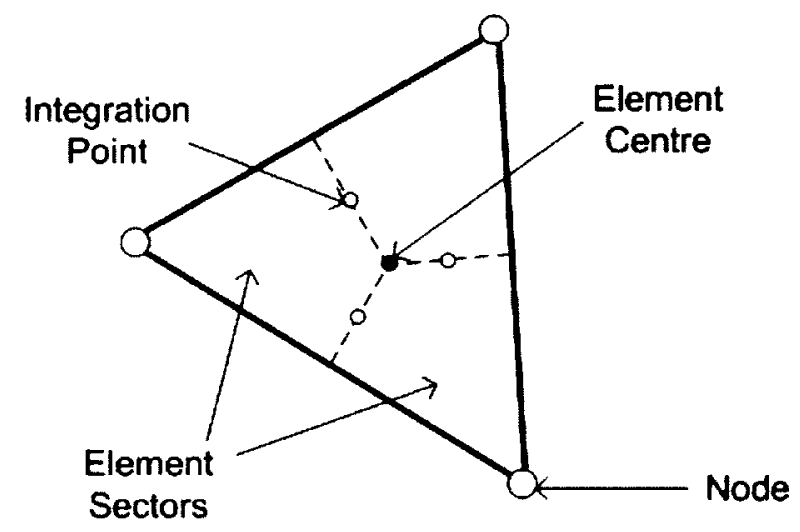

Figure 3.2: Mesh element (adapted from ANSYS, 2009)

Volume integrals are converted to a discrete form within their respective element sectors as shown in Figure 3.2 and then integrated over all the sectors in the control volume to which they belong. Surface integrals are discretized at the integration points ( $i p)$ and then distributed to the adjacent control volumes. The discretization of the Eqns. 3.5, 3.6 and 3.7 leads to the following forms:

$$
\begin{gathered}
v \frac{\Delta(\rho)}{\Delta t}+\sum_{i p}\left(\rho u_{j} \Delta n_{j}\right)_{i p}=0 \\
v \frac{\Delta\left(\rho u_{i}\right)}{\Delta t}+\sum_{i p}\left(\rho u_{j} \Delta n_{j}\right)_{i p}\left(u_{i}\right)_{i p}=\sum_{i p}\left(p \Delta n_{i}\right)_{i p}+\sum_{i p}\left(\tau_{i j} \Delta n_{j}\right)_{i p} \\
V \frac{\Delta\left(\rho h_{0}\right)}{\Delta t}-V \frac{\Delta(p)}{\Delta t}+\sum_{i p}\left(\rho u_{j} h_{0} \Delta n_{j}\right)_{i p}=\sum_{i p}\left(k \frac{\partial T}{\partial x_{j}} \Delta n_{j}\right)_{i p}+\sum_{i p}\left(u_{i} \tau_{i j} \Delta n_{j}\right)_{i p}
\end{gathered}
$$

In these equations, $V$ is the volume of the discrete control volume, $\Delta t$ is the time-step, $\Delta n_{j}$ is the discrete outward normal surface vector, ip denotes evaluation at the integration point with the $\sum_{i p}$ indicating summation over all the integration points of a control volume. The discretization of the transient terms in the above equations is performed using the second-order backward Euler scheme as shown in Eqn. 3.11 which makes use of two previous times, $n-1$ and $n-2$ : 


$$
\frac{\Delta(\phi)}{\Delta t}=\frac{1}{\Delta t}\left[\frac{3}{2} \phi-2 \phi^{n-1}+\frac{1}{2} \phi^{n-2}\right]
$$

where $\phi$ is a generic variable. ANSYS CFX® uses a collocated grid technique where the solution variables are stored at the grid nodes but various terms in the equations require variables and/or their gradients to be approximated at the integration points, and this is accomplished by the use of finite-element shape functions which describe the variation of $\phi$ within an element as follows:

$$
\phi=\sum_{i=1}^{N_{\text {node }}} N_{i} \phi_{i}
$$

where $N_{i}$ is the shape function for node $i$ and $\phi_{i}$ is the value of $\phi$ at node $i$. $\sum$ represents the summation over all the nodes of an element. The properties of the shape function are as follows:

$$
\begin{gathered}
\qquad \sum_{i=1}^{N_{\text {node }}} N_{i}=1 \\
\text { At node } j, \quad N_{i}= \begin{cases}1 & i=j \\
0 & i \neq j\end{cases}
\end{gathered}
$$

Eqn. 3.14 implies that the value of $N_{i}$ at node $i$ is 1 and 0 at any other node. The shape functions are linear in terms of parametric coordinates and are also used to calculate various geometric quantities such as the integration point coordinates:

$$
x=\sum_{i=1}^{N_{\text {node }}} N_{i} x_{i} \quad y=\sum_{i=1}^{N_{\text {node }}} N_{i} y_{i} \quad z=\sum_{i=1}^{N_{\text {node }}} N_{i} z_{i}
$$




\subsubsection{Convection and Diffusion Terms}

The convection term is evaluated by estimating the value of $\phi$ at the integration points in terms of the nodal values of $\phi$ using the following relation:

$$
\phi_{i p}=\phi_{u p}+\beta \nabla \phi \cdot \Delta \vec{r}
$$

where $\phi_{u p}$ is the value of $\phi$ at the upstream node, $\Delta \vec{r}$ is the vector from the upstream node to the integration point, and the value of $\beta$ varies from 0 to 1 . A value of $\beta=0$ implies first order upwind differencing scheme which is known to be robust but subject to significant numerical diffusion and $\beta=1$ represents the central differencing scheme that is susceptible to numerical dispersion. For the simulations in this research, the value of $\beta=1$ is used which yields a discretization for the convection terms that is formally second-order accurate on a uniform grid.

The diffusion terms are treated with the use of shape functions with spatial derivatives at an integration point in a particular spatial direction evaluated as follows:

$$
\left.\frac{\partial \phi}{\partial x_{i}}\right|_{i p}=\left.\sum_{n} \frac{\partial N_{n}}{\partial x_{i}}\right|_{i p} \phi_{n}
$$

The summation is over all the shape functions for the element and the derivatives of the shape functions in terms of Cartesian coordinates can be expressed in terms of the localcoordinate derivatives using the Jacobian transformation matrix as:

$$
\left[\begin{array}{l}
\frac{\partial N}{\partial x} \\
\frac{\partial N}{\partial y} \\
\frac{\partial N}{\partial z}
\end{array}\right]=\left[\begin{array}{lll}
\frac{\partial x}{\partial s} & \frac{\partial y}{\partial s} & \frac{\partial z}{\partial s} \\
\frac{\partial x}{\partial t} & \frac{\partial y}{\partial t} & \frac{\partial z}{\partial t} \\
\frac{\partial x}{\partial u} & \frac{\partial y}{\partial u} & \frac{\partial z}{\partial u}
\end{array}\right]^{-1}\left[\begin{array}{l}
\frac{\partial N}{\partial s} \\
\frac{\partial N}{\partial t} \\
\frac{\partial N}{\partial u}
\end{array}\right]
$$

where $s, t$ and $u$ denote the local (element-based) coordinates. 


\subsubsection{The Coupled Solver}

The application of the finite-volume method to all the elements in the domain leads to a set of linearized equations that can be summarized as follows:

$$
\begin{gathered}
\sum_{n b_{i}} a_{i}^{n b} \phi_{i}^{n b}=b_{i} \\
a_{i}^{n b}=\left[\begin{array}{llll}
a_{u u} & a_{u v} & a_{u w} & a_{u p} \\
a_{v u} & a_{v v} & a_{v w} & a_{v p} \\
a_{w u} & a_{w v} & a_{w w} & a_{w p} \\
a_{p u} & a_{p v} & a_{p w} & a_{p p}
\end{array}\right]_{i} \\
\phi_{i}{ }^{n b}=\left[\begin{array}{c}
u \\
v \\
w \\
p
\end{array}\right]_{i}^{n b} \text { and } \quad b_{i}=\left[\begin{array}{l}
b_{u} \\
b_{v} \\
b_{w} \\
b_{p}
\end{array}\right]_{i}^{n b}
\end{gathered}
$$

where $\phi$ is the solution vector, $a$ and $b$ represent the coefficients of the left- and righthand sides of the equation, $i$ is a control-volume or node-number index, and $n b$ refers to the neighbouring nodes of the central node (i). ANSYS CFX® uses a coupled solver, which solves for the variables $(u, v, w, p)$ as a single system with a fully implicit discretization of the governing equations at any given time-step. Thus, for the mass and momentum equations, $a_{i}^{n b}, \phi_{i}^{n b}$ and $b_{i}$ are matrices as shown in Eqns. 3.20 and 3.21 but for a scalar equation (such as the total enthalpy equation), $a_{i}{ }^{n b}, \phi_{i}{ }^{n b}$ and $b_{i}$ would each be single numbers.

The linearized system of discrete equations can be represented by a general form:

$$
[A][\phi]=[b]
$$

This equation is solved iteratively using the Incomplete Lower Upper (ILU) factorization technique where several iterations are performed to obtain a converged solution. Starting 
with an approximate solution $\phi^{k}$, the solution can be improved by a correction term $\phi^{\prime}$ to obtain $\phi^{k+1}$ as:

$$
\phi^{k+1}=\phi^{k}+\phi^{\prime}
$$

where $\phi^{\prime}$ is a solution of the following equation:

$$
A \phi^{\prime}=r^{k}
$$

The term $r^{k}$ is called the residual which is used as a criterion to assess the convergence of the overall solution, and it is given as:

$$
r^{k}=b-A \phi^{k}
$$

Several iterations using this procedure reduce the residual and the exact solution is approached. For the simulations in this study, the continuity, momentum and energy equations are converged at each time-step within 5 iterations yielding root mean square residuals on the order of $10^{-5}$.

In order to accelerate the convergence rates of the solution algorithm and thus reduce the number of iterations and computer time required for a converged solution, a multigrid scheme is employed. ANSYS CFX ${ }^{\circledR}$ uses an Algebraic Multigrid (AMG) technique in which iterations are cycled between fine and coarser grids constructed indirectly by algebraic manipulation of the system of linearized equations being solved. Calculations corresponding to the fine mesh reduce the shorter wavelength errors of the size of the mesh spacing while longer wavelength errors are efficiently dealt with on the coarser grids. A discrete system of equations for a coarse mesh is obtained by summing the fine mesh equations based on the merging of the original finer control volumes to create coarser ones. This is the Additive Correction (AC) technique of Hutchinson and Raithby (1986) that is used by the AMG method in this solver. More information about 
this technique can be obtained from the ANSYS CFX ${ }^{\circledR}$ solver theory guide (ANSYS, 2009).

\subsection{Computational Method}

The direct numerical simulations performed in this research are used to study the effect of streamwise convex surface curvature on the coherent structures in a zeropressure-gradient boundary layer when the flow is transitioning as well as when it is fully turbulent. In order to characterize the effect of curvature, the convex-surface simulations are compared to flat-surface simulations in similar flow environments. The large number of nodes required for these DNS studies is managed by the method of parallel processing where the computational domain used for the test cases is divided into smaller partitions and each partition is handled by a different processor. A single processor manages the flow of data between these processes and ensures solution connectivity between them. The ANSYS CFX ${ }^{\circledR}$ solver makes use of the MeTiS partitioning method (Karypis and Kumar, 1995). For the present simulations, a cluster with 20 Intel $\mathbb{R}$ L5410 Xeon $\mathbb{B}$ processors with $8 \mathrm{~GB}$ RAM dedicated to each of the processors is used. The test cases are summarized in Table 3.1; Case 1 and Case 2 require approximately 9000 CPU hours and Case 3 and Case 4 require approximately $28000 \mathrm{CPU}$ hours to run.

\subsubsection{Test Cases}

The simulations performed in this research with the relevant parameters are summarized in Table 3.1, where $\delta / R$ is the ratio of the boundary layer thickness to the radius of curvature of the surface, $q / G$ is the wall heat flux-to-mass flux ratio in the 
momentum boundary layer, $p$ is the nominal static pressure and $R e_{\tau}=u_{\tau} \delta / \nu=330$ is the target Reynolds number. Two of the simulations employ air at standard sea level conditions and the remaining simulations utilize water above the critical pressure as the working fluid. The simulations that utilize air as the working fluid are used to validate the numerical approach and to establish the effect of convex curvature on the coherent flow structures of turbulence in the absence of thermophysical property variations. The boundary layers developed using water as the working fluid have a wall-normal temperature gradient imposed on the flow field by means of a uniformly applied wall heat flux. The value of the heat flux in conjunction with the mass flux in the boundary layer corresponds to the simulation of a flat-surface case by Azih et al. (2012) in which improved heat transfer was observed.

Table 3.1: Simulation parameters.

\begin{tabular}{|c|c|c|c|c|c|c|c|}
\hline $\begin{array}{c}\text { Test } \\
\text { cases }\end{array}$ & $\begin{array}{c}\text { Surface } \\
\text { geometry }\end{array}$ & $\boldsymbol{\delta} / \boldsymbol{R}$ & $\begin{array}{c}\text { Working } \\
\text { fluid }\end{array}$ & $\begin{array}{c}\boldsymbol{q} / \boldsymbol{G} \\
(\mathbf{k J} / \mathbf{k g})\end{array}$ & $\begin{array}{c}\text { Thermodynamic } \\
\text { state }\end{array}$ & $\begin{array}{c}\boldsymbol{p} \\
(\mathbf{M P a})\end{array}$ & $\boldsymbol{R} \boldsymbol{e}_{\boldsymbol{\tau}}$ \\
\hline Case 1 & Flat & 0 & Air & 0 & Ideal gas & 0.1 & 330 \\
\hline Case 2 & Convex & 0.0076 & Air & 0 & Ideal gas & 0.1 & 330 \\
\hline Case 3 & Convex & 0.0017 & Water & 0.9 & Supercritical & 24 & 330 \\
\hline Case 4 & Convex & 0.0400 & Water & 0.9 & Supercritical & 24 & 330 \\
\hline
\end{tabular}

\subsubsection{Computational Domain}

The computational domain is shown in Figure 3.3, and the corresponding dimensions are specified in Table 3.2 for each test case, with $\delta$ being the boundary layer thickness corresponding to the respective test case. The variables $L x, L y$ and $L z$ denote the dimensions in the streamwise, wall-normal and spanwise directions for both the flat- and 
convex-surface cases with the subscript "fr" indicating finely-resolved region and superscript "+" indicating normalization by the wall variables. The domains are described in more detail in the following sub-sections.

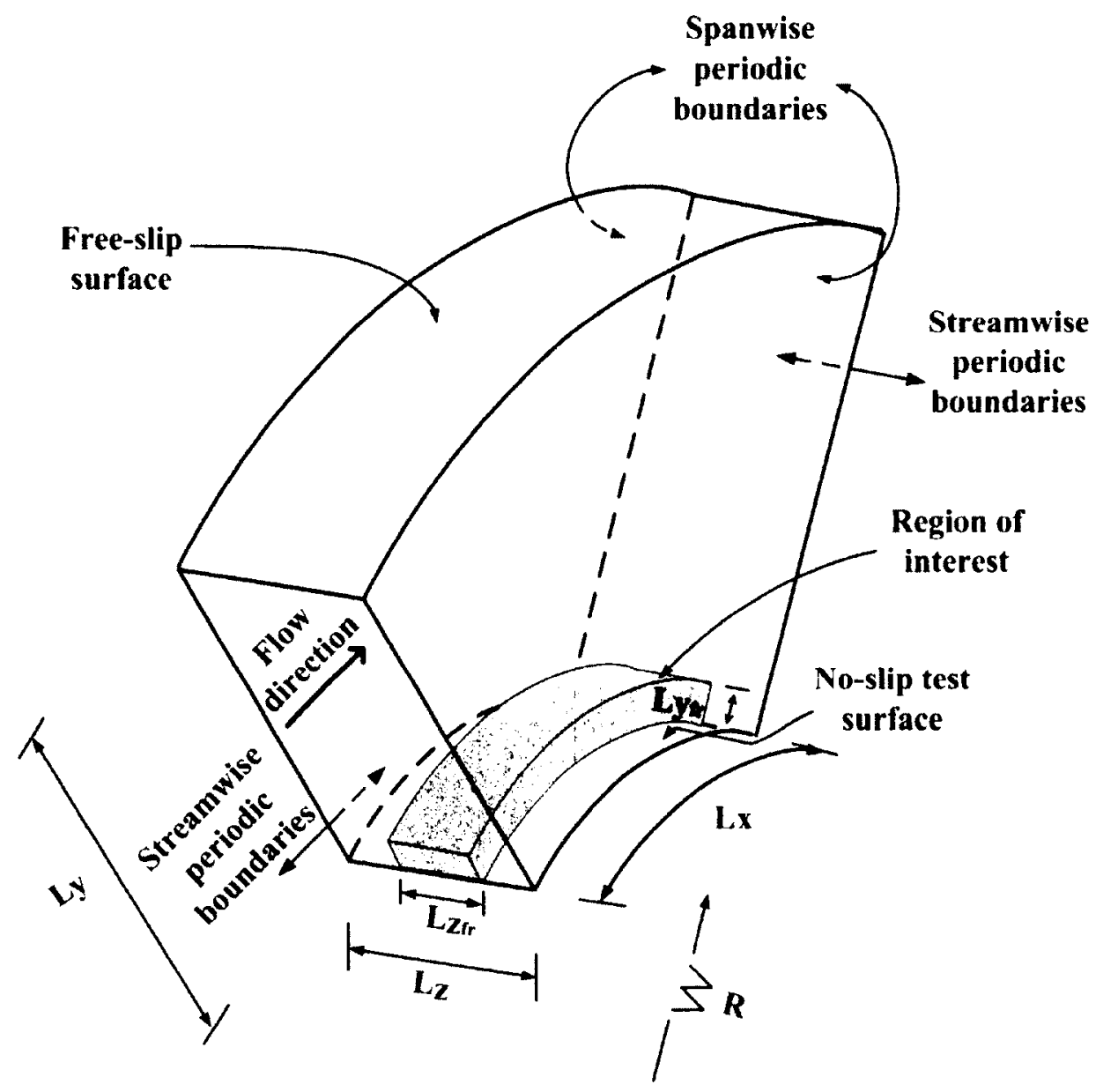

Figure 3.3: Schematic of the computational domain shown with a geometry representative of the surface curvature of Case 4.

Table 3.2: Computational domain parameters.

\begin{tabular}{|c|c|c|c|c|c|}
\hline Test case & $\boldsymbol{L} \boldsymbol{x}\left(\boldsymbol{L} \boldsymbol{x}^{+}\right)$ & $\boldsymbol{L} \boldsymbol{y}\left(\boldsymbol{L y}^{+}\right)$ & $\boldsymbol{L}_{z}\left(\boldsymbol{L}_{z}^{+}\right)$ & $\boldsymbol{L}_{f_{r}}\left(\boldsymbol{L}_{y_{f r}}{ }^{+}\right)$ & $\boldsymbol{L}_{z_{f r}}\left(\boldsymbol{L}_{z_{f}}{ }^{+}\right)$ \\
\hline Case 1 & $9 \delta(3000)$ & $11 \delta(3667)$ & $4 \delta(1333)$ & $1.7 \delta(567)$ & $1.8 \delta(600)$ \\
\hline Case 2 & $9 \delta(3000)$ & $11 \delta(3667)$ & $4 \delta(1333)$ & $1.7 \delta(567)$ & $1.8 \delta(600)$ \\
\hline Case 3 & $18 \delta(6000)$ & $22 \delta(7333)$ & $8 \delta(2667)$ & $3.4 \delta(1133)$ & $3.6 \delta(1200)$ \\
\hline Case 4 & $18 \delta(6000)$ & $22 \delta(7333)$ & $8 \delta(2667)$ & $3.4 \delta(1133)$ & $3.6 \delta(1200)$ \\
\hline
\end{tabular}




\subsubsection{Computational Domain for the Baseline Flat-Surface Case}

The simulation parameters for the flat-surface Case 1 are set to be comparable to the experiments of Adrian et al. (2000) who used particle image velocimetry (PIV) to study the development of coherent flow structures in a zero-pressure-gradient flat-surface turbulent boundary layer. These experiments identified hairpin-vortex packets as the largest coherent structures in the boundary layer, and accordingly the streamwise length of the present computational domain is sized at $9 \delta$. This length corresponds to $L x^{+}=3000$, which according to Moin and Mahesh (1998) is more than adequate to accurately resolve the turbulence statistics in the inner region of the boundary layer corresponding to $L y^{+}<40$. The domain has a spanwise width of $L z^{+}=1333$, which is sufficient to capture the three dimensional behaviour of boundary-layer turbulence at the centre of the domain without artificial influence of the periodic side boundaries. The spanwise width of the hairpin-vortex wave packets is determined to be $\Delta z^{+} \approx 100$ which is the average spacing of streaks as is observed in several DNS studies such as those of Zhou et al. (1999), Hidalgo et al. (2006) and Sabatino and Smith (2008). The well-resolved region (region of interest) identified with a shaded volume in Figure 3.3 has a spanwise width of $L z_{\mathrm{fr}}{ }^{+}=600$ for Case 1 which is sufficient for the evolution of multiple wave packets along the span. The wallnormal height of the region of interest is $1.7 \delta$, which is sufficient to include the instantaneous height of the coherent structures in an unconstrained manner (Pope, 2000). 


\subsubsection{Computational Domain for the Streamwise Convex Surface Curvature Cases}

In the present study, the simulations utilizing supercritical water as the working fluid (Cases 3 and 4) are performed to study the effects of streamwise convex surface curvature on the convective heat transfer mechanism in turbulent flow over nuclear rods in fuel bundles. This is because the wire-wrap spacers on nuclear fuel rods simultaneously induce transverse velocities and impose streamwise convex surface curvature on the flow as it develops over the surface of each fuel rod in the bundle (see Chapter 2 for a detailed description of wire-wrap spacers). In order to create the streamwise convex curvature for the present simulations, the flat surface of the baseline case is deformed at a constant radius such that the arc length of the convex surface equals the streamwise length of the flat surface. For Case 4 , the value of $\delta / R=0.04$ is comparable to the streamwise curvature induced by a wire-wrap with a pitch of $25 \mathrm{~cm}$ spirally wound around a nuclear-reactor fuel rod with a diameter of $10 \mathrm{~mm}$ and length of $50 \mathrm{~cm}$. As discussed in Chapter 2, the effect of streamwise convex curvature on a turbulent boundary layer is characterized by the ratio $\delta / R$ and $\delta / R=0.04$ in the present case is considered to be strong curvature based on experiments performed in the past with turbulent boundary layers on convex surfaces that have $\delta / R$ in a similar range (So and Mellor, 1973; Gillis and Johnston, 1983). Since convex curvature is known to exhibit a stabilizing influence on the boundary layer with the critical Reynolds number increasing with stronger curvature, it was decided to initially run simulations with $\delta / R=0.0076$ (Case 2 using subcritical air) and $\delta / R=0.0017$ (Case 3 using supercritical water) which are 
considered to be in the mild curvature range. The domain dimensions of the curved cases are shown in Figure 3.3 and Table 3.2.

\subsubsection{Spatial and Temporal Grid Resolution}

The distribution of nodes is an important factor in DNS of wall-bounded turbulent flows as the smallest scales of the eddies need to be resolved spatially and temporally (Kleiser and Zang, 1991). The smallest spatial and temporal scales in wall-bounded turbulent flows are of the order of Kolmogorov length and time scales given as:

$$
\begin{aligned}
& \eta=\left(\frac{v^{3}}{\epsilon}\right)^{\frac{1}{4}} \\
& t_{k}=\left(\frac{v}{\epsilon}\right)^{\frac{1}{2}}
\end{aligned}
$$

where $v$ is the kinematic viscosity and $\epsilon$ is the turbulence kinetic energy dissipation rate. For wall-bounded turbulent boundary layers, the Kolmogorov length scale is of the order of the viscous length scale reaching a minimum value of $\eta^{+} \approx 2$ close to the wall. According to Moin and Mahesh (1998), the resolved spatial scale is to be of the order of $\eta$, but need not be equal to $\eta$ because experimental observations have shown that the smallest scales in turbulent boundary layers are notably greater than $\eta$. DNS of channel flows such as the study of Kim et al. (1987) and DNS of boundary-layer flows such as the study of Spalart (1988) have shown excellent agreement with experimental results without spatially resolving down to the Kolmogorov length scale.

Friedrich et al. (2001) note that for numerical stability the time-step size should be notably below the $t_{k}$ value for explicit schemes, but for implicit schemes such as the one used for the simulations in the present study, the viscous time scale can be used which is 
also of the order of $t_{k}$. However, Friedrich et al. (2001) recommend a conservative value for the time-step size to sustain turbulence in wall-bounded turbulent boundary layers that is almost an order of magnitude less than $t_{k}$. This value, calculated according to the following equation, is used for the simulations in this study.

$$
\Delta t=0.2 \frac{v}{u_{\tau}^{2}}
$$

\subsubsection{Spatial and Temporal Resolution for the Baseline Flat-Surface Case}

The structured spatial grid mapped onto the computational domain of the baseline case (Case 1) consists of a finely-resolved region (the region of interest), surrounded by a region in which the grid cell size is increased with distance from the finely-resolved region until the boundaries of the domain are reached. The region of interest is populated with 504 nodes in the streamwise, 135 nodes in the wall-normal and 103 nodes in the spanwise direction, respectively. In the streamwise and spanwise directions, the grid nodes are distributed uniformly with spacings of $\Delta x^{+}=6$ and $\Delta z^{+}=6$. The $y^{+}$value of the first node from the wall is 0.75 and this value is increased with distance from the wall according to the following equation that corresponds to the Kolmogorov scale in the loglaw region (Stanislas et al., 2008):

$$
\eta^{+}=\left(\kappa y^{+}\right)^{\frac{1}{4}}
$$

where, $\kappa=0.41$ is the Von-Karman constant. Above the log-law region. the node spacing is kept constant until $1.7 \delta$, after which it is increased at a constant rate of $12 \%$ until the domain height is reached.

The time-step size used is calculated according to Eqn. 3.28 and has a value of $\Delta t^{+}=0.11$ where $\Delta t^{+}=\Delta t u_{\mathrm{r}}^{2} / v$, and is less than $\Delta t^{+}=0.2$ which is recommended by Friedrich 
et al. (2001) for an accurate prediction of turbulence statistics and to sustain turbulence in the flow. The grid for the flat-surface case is summarized in Table 3.3.

Table 3.3: Computational spatial grid resolution in the finely-resolved region.

\begin{tabular}{|c|c|c|c|}
\hline Test case & $\boldsymbol{\Delta x}^{+}$ & $\boldsymbol{y y}^{+}(\mathbf{a t} \boldsymbol{y}=\mathbf{0})$ & $\boldsymbol{\Delta z}^{+}$ \\
\hline Case 1 & 6 & 0.75 & 6 \\
\hline Case 2 & 6 & 0.75 & 6 \\
\hline Case 3 & 12 & 0.67 & 12 \\
\hline Case 4 & 12 & 0.67 & 12 \\
\hline
\end{tabular}

\subsubsection{Spatial and Temporal Resolution for the Convex-Surface Cases}

The spatial grid resolutions for the convex-surface cases (Cases 2, 3 and 4) are shown in Table 3.3. For the convex-surface cases, the turbulence activity is reduced due to the stabilizing influence of streamwise convex curvature (Gillis and Johnston, 1983) and thus the computational grid sizing for the flat-surface case yields a conservative grid for the convex-surface cases. Thus, the region of interest in the convex-surface domain is also populated with 504, 135 and 103 nodes in the streamwise, wall-normal and spanwise directions, respectively, with similar $\Delta x^{+}, \Delta z^{+}$and $\Delta y^{+}$values for Case 2. A grid resolution study was conducted for the flat-surface case with $\Delta x^{+}$and $\Delta z^{+}$values of 3,5 , 10 and 19 by Azih et al. (2012) and the results are listed in Table 3.4. The results indicate that a grid resolution with $\Delta x^{+} \approx 10$ and $\Delta z^{+} \approx 10$ is adequate to capture the turbulence hydrodynamics. The grid resolution of $\Delta x^{+}=12$ and $\Delta z^{+}=12$ was chosen for Cases 3 and 4 to ensure that these simulations have reasonable computing times while accurately capturing the spatial and temporal development of turbulence. Also, when the flow is 
heated, the node spacing is adjusted in the wall-normal direction to capture the variations in the thermal field according to:

$$
\Delta y_{\text {thermal }}=\Delta y_{\text {momentum }}(P r)^{-1 / 3}
$$

where, $\Delta y_{\text {thermal }}$ is the node spacing requirement for the thermal boundary layer, $\Delta y_{\text {momentum }}$ is the node spacing requirement for the momentum boundary layer and $\operatorname{Pr}$ is the Prandtl number of the flow (Redjem-Saad et al., 2007). The Pr value for Cases 3 and 4 remains greater than unity, implying that the thermal boundary layer is contained within the momentum boundary layer in the region of interest.

In terms of the temporal resolution, a value of $\Delta t^{+}=0.11$ is used for Case 2 simulated with air as the working fluid and a value of $\Delta t^{+}=0.013$ is used for Cases 3 and 4 simulated with supercritical water as the working fluid. This small value of $\Delta t^{+}$is used for Cases 3 and 4 to counter numerically destabilizing effects of significant spatial gradients in thermophysical properties of supercritical water.

Table 3.4: Comparison of mean velocity profile data for various spatial grid resolutions for a flat-surface simulation by Azih et al. (2012).

\begin{tabular}{|c|c|c|c|}
\hline Grid & $\Delta \boldsymbol{x}^{+}$ & $\Delta \boldsymbol{z}^{+}$ & $\begin{array}{c}\text { Maximum difference in velocity } \\
\text { with reference to Grid 4 }\end{array}$ \\
\hline Grid 1 & 19 & 19 & $10 \%$ \\
\hline Grid 2 & 10 & 10 & $3.5 \%$ \\
\hline Grid 3 & 5 & 5 & $0.21 \%$ \\
\hline Grid 4 & 3 & 3 & $0 \%$ \\
\hline
\end{tabular}




\subsubsection{Initial and Boundary Conditions}

The initial and boundary conditions determine the spatial and temporal evolution of the flow in a computational study. The boundary conditions are indicated in Figure 3.3. A no-slip condition is applied to the lower boundary of the computational domain to simulate the surface over which the boundary layer is to develop. A free-slip boundary condition with zero shear-stress and boundary-normal velocity is imposed on the computational domain boundary that is facing the wall boundary. Periodic boundary conditions are applied in the streamwise and spanwise directions, and thus the temporal development of the boundary layer occurs in a spatially uniform manner. Periodic boundary conditions are applied in ANSYS CFX® by mapping the variables at the nodes on the outflow plane to the corresponding nodes at the inflow plane. For the baseline case, this is accomplished by using translational periodic boundaries as they are parallel but for the curved domains, rotational periodic boundaries are used by specifying the corresponding axis of rotation.

The baseline unheated flat-surface case (Case 1) is initialized with a spatiallyuniform velocity of $1.6 \mathrm{~m} / \mathrm{s}$ at standard sea level conditions. The unheated convex-surface case (Case 2) is initialized with a linear radial distribution of streamwise velocity such that the initial velocity at the first node off the wall for the curved-surface case matches the freestream velocity for the flat-surface case. The linear radial distribution is employed to yield a solid-body-rotation type velocity field, and thus zero shear in the freestream fluid. The cases involving air at subcritical state as the working fluid involve no heat transfer across the domain boundaries and a uniform temperature distribution of $300 \mathrm{~K}$ is 
used throughout the domain. The heated cases, which use supercritical water as the working fluid, are initialized with a linear radial distribution of streamwise velocity such that the initial Reynolds number based on the streamwise velocity at the first node off the wall and the viscosity at the initial thermodynamic state is equivalent for the unheated and heated convex-surface cases. The initial thermodynamic state of the fluid for the heated cases is defined by the temperature and pressure values of $633 \mathrm{~K}$ and $24 \mathrm{MPa}$, respectively. All curved-surface cases are initialized with a parabolic pressure distribution in the radial direction to yield radial equilibrium for a linear radial distribution of streamwise velocity. Body forces are not specified to isolate the heattransfer variations that result from local thermophysical property gradients and streamwise surface curvature, from mechanisms due to buoyancy interactions within the boundary layer.

\subsubsection{Forced Boundary Layer Disturbances}

To ensure that the flow for the unheated cases reach a fully turbulent state at the target Reynolds number, transition from laminar to turbulent flow state is promoted at a lower Reynolds number value for Cases 1 and 2 by the use of a mass-source disturbance located in close proximity to the no-slip surface. The mass flow rate introduced at the disturbance can be varied according to the following equation:

$$
\dot{m}=\Delta x_{\text {node }} \Delta z_{\text {node }} \rho k u_{\infty}
$$

In this equation, $\dot{m}$ is the mass flow rate, $\Delta x_{\text {node }}$ and $\Delta z_{\text {node }}$ are the dimensions of a grid element in the $x$ and $z$ directions, respectively, $u_{\infty}$ is the freestream velocity and $k$ is a constant that can be varied to produce a disturbance mass flow rate with a velocity that is 
a fraction or multiple of the freestream velocity. The use of the value of $k$ will be elaborated upon in Chapter 4 of this document. The disturbance is introduced at a location centred between the spanwise periodic boundaries and 500 wall units downstream of the inflow streamwise periodic boundary.

For Cases 3 and 4, the variations in the flow hydrodynamics that result from the strong temperature dependence of the thermophysical properties provide disturbances of sufficient magnitude and wavelength such that the flow is fully turbulent at the target Reynolds number. 


\section{Chapter: Results and Discussion}

This chapter begins with a validation of the numerical approach employed to conduct the simulations listed in Table 3.1 followed by a study of the growth of forced disturbances in a boundary layer developing over a streamwise mildly-curved convex surface, Case 2, and its comparison with the flat-surface, Case 1. Next, the characteristics of the coherent structures in the fully turbulent boundary layer are compared for Cases 1 and 2 followed by a comparison of the development of such structures over streamwise mildly-curved (Case 3) and strongly-curved (Case 4) convex surfaces, both of which are uniformly heated from the test surface. Table 3.1 on page 51 shows the simulation parameters employed in the present study.

Before presenting the results for the cases listed in Table 3.1, it should be noted that a section of these results have been the subject of a paper by Dave et al., 2012 currently under review in the Journal of Nuclear Engineering and Design. Besides the thesis supervisor, the contribution of the second author in the paper, Chukwudi Azih, who is a $\mathrm{PhD}$ candidate in the present author's research group, is acknowledged in the following areas:

- Creation of the grid for the flat-surface Case 1, which was subsequently adapted for the remaining cases listed in Table 3.1

- Conducting a grid-resolution study for the flat-surface case

- Assistance in the general interpretation of simulation data for the cases listed in Table 3.1 
In the following sections, variables used for normalization are evaluated at the target Reynolds number of $R e_{\tau}=u_{\tau} \delta / v=330$, where $v$ is the kinematic viscosity at the wall and $u_{\tau}$ is the friction velocity. Unless otherwise specified, all quantities are normalized using mean values of the wall variables, $u_{\mathrm{\tau}}$ and $v$. Normalization with $u_{\tau}$ and $v$ is indicated with a "+" superscript and the thermophysical property values are evaluated at the wall temperature. The boundary layer thickness for Case 1 is calculated based on the location where the mean streamwise velocity is $99 \%$ of the freestream velocity. Since the curvedsurface cases are initialized with a radially linear distribution of streamwise velocity, the edge velocity of the boundary layer for these cases is evaluated at the point where the slope of the mean streamwise velocity profile matches the slope of the initial radial distribution of velocity. The mean-flow property values at a given wall-normal location are obtained by spatial averaging in the spanwise and streamwise directions. This is made possible by the use of streamwise- and spanwise-periodic boundary conditions which enforces temporal development of the boundary layer in a spatially uniform manner.

\subsection{Validation of the Numerical Method}

Validation of the numerical approach is performed by comparing the results obtained from the flat-surface simulation (Case 1) with published literature involving comparable flow conditions and geometry. In Figure 4.1, the mean velocity profile for Case 1 is compared to the DNS data of Spalart (1988) and Wu and Moin (2009), who simulated the development of a turbulent boundary layer over a flat surface. Spalart (1988) solved the governing equations using a spectral method based on Fourier series in the directions parallel to the wall and Jacobi polynomials in the wall-normal direction in a 
computational domain with periodic streamwise and spanwise boundaries. Wu and Moin (2009) used a second-order Crank-Nicolson scheme for implicit terms and a third-order Runge-Kutta scheme for explicit terms, with periodic boundary conditions in the spanwise direction. Both authors have thoroughly verified the accuracy of their DNS datasets against published experimental data, and in the case of Spalart (1988), more recent experimental studies such as that of Adrian et al. (2000) have further verified the accuracy of this DNS data. The computed mean velocity profile for Case 1 shows excellent agreement with both published datasets especially in the near-wall region of $y^{+}<40$. Figure 4.2 compares the corresponding root mean square (rms) of velocity fluctuations with the data of Spalart (1988) and Wu and Moin (2010) and shows satisfactory agreement in all three velocity components with a maximum deviation of less than 7\%. Honkan and Andreopoulos (1997) reveal that there is some discrepancy in published literature when they compare the rms of velocity fluctuations obtained by different researchers at different Reynolds numbers. They find that the maximum value of $u_{r m s}^{\prime+}$ ranges from 2.3 to 2.9 , the plateau in $v_{r m s}^{\prime+}$ ranges from 0.8 to 1.4 and that in $w_{r m s}^{\prime+}$ ranges from 1.4 to 1.6 . The rms of velocity fluctuations in the present case are therefore well within the range of values in published literature. Further validation of the numerical method is shown in Figure 4.3 which compares the production and dissipation rates from the budget of the Reynolds normal stresses for Case 1 with the data of Spalart (1988), indicating a reasonable agreement in both production and dissipation rates. The maximum value of $u_{r m s}^{\prime^{+}}$and production occurs at $y^{+} \approx 11$ for the present simulation and this value is within the narrow range of values for the peak $u_{r m s}^{\prime+}$ and Reynolds normal stress production (Spalart, 1988; Honkan and Andreopoulos, 1997). These favourable 
comparisons confirm the suitability of the present computational algorithm, spatial and temporal resolutions, and computational domain boundary treatments.

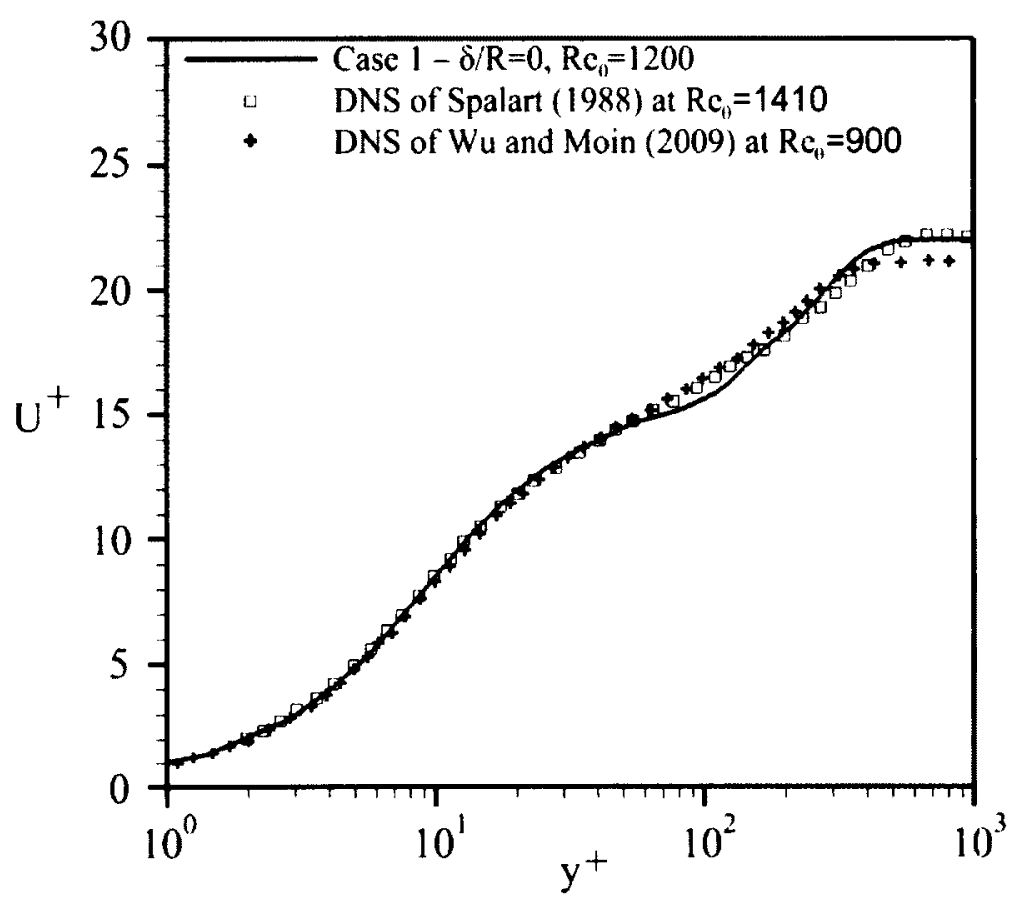

Figure 4.1: Comparison of mean velocity profiles for Case 1 with the flat-surface DNS data of Spalart (1988) and Wu and Moin (2009). 


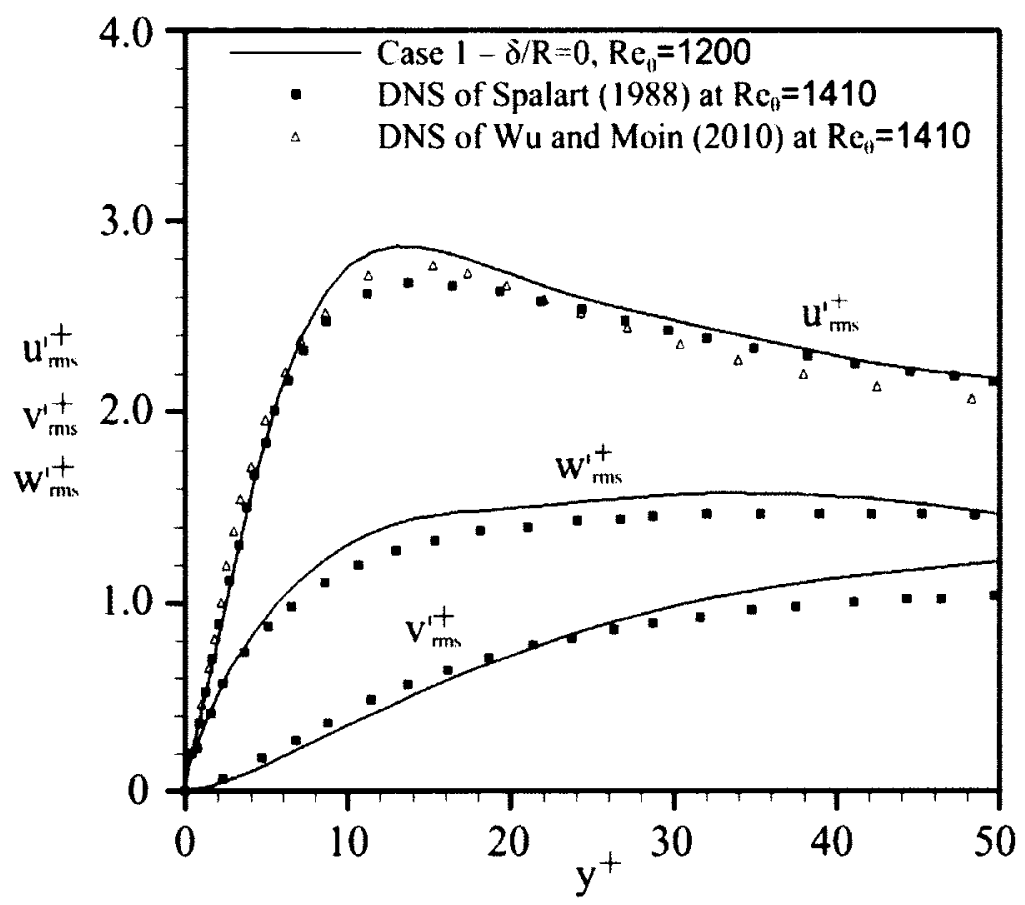

Figure 4.2: Comparison of fluctuating velocity fields for Case 1 with the flat-surface DNS data of Spalart (1988) and Wu and Moin (2010).

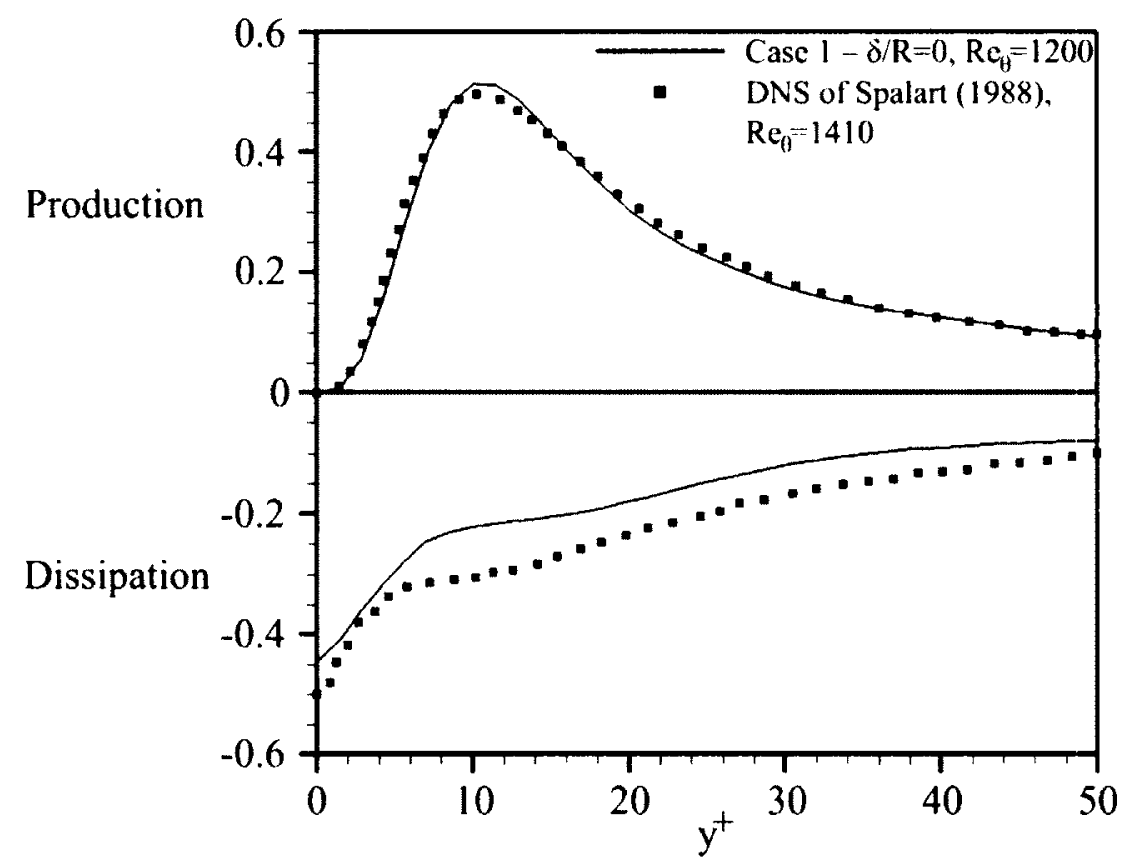

Figure 4.3: Comparison of production and dissipation rates from the budget of the Reynolds normal stresses for Case 1 with the flat-surface DNS data of Spalart (1988). 


\subsection{Effect of Streamwise Convex Surface Curvature on the Initial Evolution of \\ Forced Boundary Layer Disturbances}

This section describes the initial evolution of a disturbance in the boundary layer developing over a mildly-curved streamwise convex surface (Case 2) and compares the growth of the same disturbance in the flat-surface case (Case 1). The introduction of the disturbance is to ensure that the flow for the unheated cases (Cases 1 and 2) reach a fully turbulent state at the target Reynolds number. For Cases 3 and 4, the variations in the flow hydrodynamics that result from the strong temperature dependence of the thermophysical properties provide disturbances of sufficient magnitude and wavelength such that the flow is fully turbulent at the target Reynolds number. An unheated case for the strongly-curved streamwise convex surface is unable to reach a fully turbulent state at the target Reynolds number due to its strong stabilizing effects and a higher critical Reynolds number. The strongly-curved convex surface causes a dissipation of the introduced disturbances and relaminarizing of the flow which is shown in Appendix B.1.

Although the objective of the present research is to characterize the effect of streamwise convex surface curvature on turbulent boundary layers, the initial evolution of the disturbance is described as many of the developmental features are replicated in fully turbulent boundary layers. It should be noted here that an initial disturbance always evolves into a qualitatively similar hairpin-like structure irrespective of the nature of the disturbance introduced in the boundary layer. For example, in the experiments of Acarlar and Smith (1987b) and in the simulations of Zhou et al. (1999), Wu and Moin (2009) among others who use different techniques to introduce disturbances in a "clean" background flow (the methods used by these researchers are stated in Chapter 2 of this 
document), such a qualitative variation of disturbance results in the formation of the same general structure in the form of a hairpin-like vortex. In the present study, the disturbance is created with a point source placed near the wall (a detailed description on the working of the point source is presented in Section 3.3.5) that introduces mass in the wall-normal direction into the boundary layer at a prescribed velocity. The velocity at which the mass is injected is varied from $25 \%$ to $125 \%$ of the freestream velocity and the duration is varied from $\Delta t^{+}=12.5$ to $100\left(\Delta t^{+}=\Delta t u_{\tau}^{2} / v\right)$. For the mass-injection disturbance velocity and duration ranges studied in the present simulations, the disturbance develops into a hairpin-like vortex but the strength of the vortex varies with the strength of the disturbance affecting the further growth and development of the initial hairpin-like vortex. For example, a relatively weaker disturbance evolves in the form of a hairpin vortex but persists only for a short duration eventually dissipating without creating subsequent generations of hairpin vortices. It is not the objective of the present research to compare the growth of disturbances with different mass-injection velocity and duration ranges or determine a threshold value for the development of generations of hairpin vortices; rather, this study was conducted to determine that the state of the fully turbulent boundary layer is similar irrespective of the initial disturbance. A comparison of boundary layers on the convex surface Case 2 that reached a fully turbulent state using two different mass-injection disturbance duration ranges is shown in Appendix B.2. The mass-injection disturbance velocity and duration used to attain a fully turbulent boundary layer for the present cases (Cases 1 and 2) is listed in the following section and the resultant turbulent boundary layer state is confirmed to be independent of the disturbance velocity and duration range used. 


\subsubsection{Evolution of the Initial Disturbance on the Mildly-Curved Streamwise Convex Surface}

The evolution of a disturbance described below is for the point source introducing mass in the wall-normal direction at a velocity that is $85 \%$ of the freestream velocity ( $k=0.85$ in Eqn. 3.31). This disturbance is introduced in a laminar base flow at $R e_{\theta}=650$ for a duration of $\Delta t^{+}=100$. The structure of the disturbance is revealed using an isosurface of the second invariant of the velocity gradient tensor $(Q)$. The velocity gradient tensor is shown in Eqn. 4.1 in the cylindrical coordinate system for clarity.

$$
V_{\text {grad_tensor }}=\left[\begin{array}{ccc}
\frac{1}{r} \frac{\partial V_{\theta}}{\partial \theta}+\frac{V_{r}}{r} & \frac{\partial V_{\theta}}{\partial r} & \frac{\partial V_{\theta}}{\partial z} \\
\frac{1}{r} \frac{\partial V_{r}}{\partial \theta}-\frac{V_{\theta}}{r} & \frac{\partial V_{r}}{\partial r} & \frac{\partial V_{r}}{\partial z} \\
\frac{1}{r} \frac{\partial V_{z}}{\partial \theta} & \frac{\partial V_{z}}{\partial r} & \frac{\partial V_{z}}{\partial z}
\end{array}\right]
$$

In Eqn. 4.1, $V_{\text {grad tensor }}$ is the velocity gradient tensor in cylindrical coordinates where $\theta$ is the angular coordinate along a curved surface, $r$ is the radial coordinate perpendicular to a curved surface and $z$ is the spanwise coordinate. $V_{\theta}, V_{r}$ and $V_{z}$ are the velocities in the tangential $(\theta)$, radial $(r)$ and spanwise directions $(z)$, respectively. The second invariant of the velocity gradient tensor can be determined from Eqn. 4.2 .

$$
Q=\frac{1}{2}\left[\left(t r\left(V_{\text {grad_tensor }}\right)\right)^{2}-\operatorname{tr}\left(V_{\text {grad_tensor }} V_{\text {grad_tensor }}\right)\right]
$$


In Eqn. 4.2, $Q$ is the second invariant of the velocity gradient tensor $\left(V_{g_{\text {rad }} \text { tensor }}\right)$ and $t r$ is the trace which is a sum of the diagonal elements of the corresponding tensor. Expanding Eqn. 4.2 yields the following form for the equation of $Q$ :

$$
\begin{gathered}
Q=V_{11} V_{22}+V_{22} V_{33}+V_{11} V_{33}-V_{12} V_{21}-V_{23} V_{32}-V_{13} V_{31} \\
V_{11} V_{22}=\left(\frac{1}{r} \frac{\partial V_{\theta}}{\partial \theta}+\frac{V_{r}}{r}\right) \frac{\partial V_{r}}{\partial r} \\
V_{22} V_{33}=\frac{\partial V_{r}}{\partial r} \frac{\partial V_{z}}{\partial z} \\
V_{11} V_{33}=\left(\frac{1}{r} \frac{\partial V_{\theta}}{\partial \theta}+\frac{V_{r}}{r}\right) \frac{\partial V_{z}}{\partial z} \\
V_{12} V_{21}=\frac{\partial V_{\theta}}{\partial r}\left(\frac{1}{r} \frac{\partial V_{r}}{\partial \theta}-\frac{V_{\theta}}{r}\right) \\
V_{23} V_{32}=\frac{\partial V_{r}}{\partial z} \frac{\partial V_{z}}{\partial r} \\
V_{13} V_{31}=\frac{\partial V_{\theta}}{\partial z}\left(\frac{1}{r} \frac{\partial V_{z}}{\partial \theta}\right)
\end{gathered}
$$

In Eqn. 4.3, $V_{i j}$ represents the entry in the particular row $(i=1,2,3)$ and column $(j=1,2,3)$ in the velocity gradient tensor form shown in Eqn. 4.1.

As the point source disturbance is activated, flow is introduced into the domain in the wall-normal direction and this disturbance flow does not initially have any streamwise momentum. This causes the main flow boundary layer fluid to decelerate as it approaches the point source forming a high pressure region upstream of the point source (Singer and Joslin, 1994). The boundary layer flow is thus deflected around the wallnormal disturbance flow which is also observed in a simulation by Brinkerhoff and Yaras (2011) in their study of a square jet ejected transversely in a laminar base flow. Due to the deceleration of the main flow boundary layer fluid upstream of the point source, a 
streamwise velocity deficit is created in that region leading to the formation of a locally inviscid unstable shear layer. This unstable shear layer rolls up to form an initial structure resembling a hairpin vortex loop as illustrated in Figure 4.4 and labelled as A. The structure is revealed using the second invariant of the velocity gradient tensor $(Q)$ coloured according to the wall-normal height using a value of $Q^{+}=0.0027\left(Q^{+}=Q v^{2} / u_{\tau}{ }^{4}\right)$, and this value is used for all subsequent figures in this section to best illustrate the features of the hairpin-like structures. All spatial coordinates are normalized by the boundary layer thickness and the time $t^{+}=0$ indicates the time at which the disturbance is first introduced into the boundary layer.

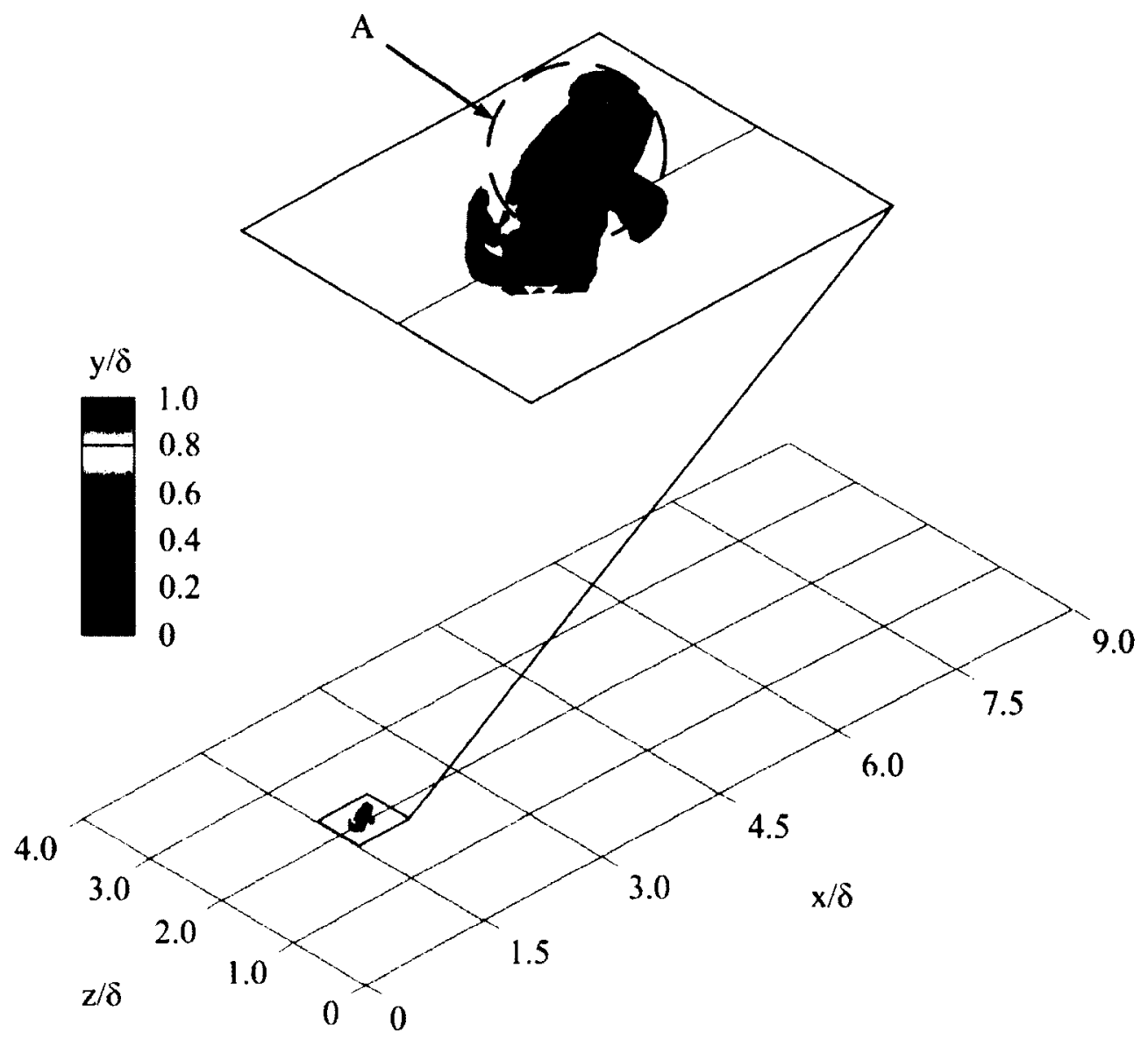

Figure 4.4: Shear layer roll-up to form an initial structure resembling a hairpin vortex loop at $t^{+}=12.5$ 
Once the initial structure is formed, it is stretched by the mean shear of the flow resulting from the velocity gradient present in the boundary layer as illustrated in Figure 4.5. In Figure 4.5, the part of the structure labelled as the "head" consists primarily of spanwise vorticity and the parts labelled as the "legs" are quasi-streamwise vortices. These quasi-streamwise vortices are counter-rotating and together with the head of the vortex, they induce a wall-normal motion of low-speed fluid away from the wall upwards and backwards in between the legs and underneath the head causing an ejection (Q2) event. On the outboard side of the structure, a wall-normal motion of high-speed fluid is induced towards the wall downward and forward causing a sweep (Q4) event. The fluid motion induced by the counter-rotating legs is shown in Figure 4.6 using the instantaneous perturbation velocity vector in a spanwise-wall-normal plane cutting through the legs of the hairpin structure. The perturbation velocity vector is calculated by subtracting the mean velocity from the instantaneous local velocities at a particular wallnormal location. Due to induction of the low-speed fluid into the high-speed region, a shear layer is created right above the initial hairpin vortex which is inviscidly-unstable due to the presence of a local inflectional point. The vorticity in this shear layer is oriented in the spanwise direction as shown in Figure 4.7. The point where the shear layer is the most unstable rolls up to form a spanwise vortex at $t^{+}=37.5$ which is visible in Figure 4.8. On its upstream side, the newly-formed spanwise vortex induces an upward velocity on the quasi-streamwise vortices in close proximity and causes a lift-up of the quasi-streamwise vortices. The spanwise vortex then connects to the lifted-up, quasistreamwise vortices to form a secondary hairpin vortex which is labelled as B in Figure 4.8(a). A similar mechanism takes place to form the tertiary hairpin vortex labelled as C 
at $t^{+}=50$ which is also clearly seen in Figure 4.8(b). On an average, a new hairpin vortex is formed every $\Delta t^{+} \approx 14$ while the disturbance source is still active. The vorticity introduced by the disturbance source into the boundary layer aligns itself with the quasistreamwise vortices which can be seen in Figure 4.8 as the legs connecting the hairpinlike vortices to the disturbance source.

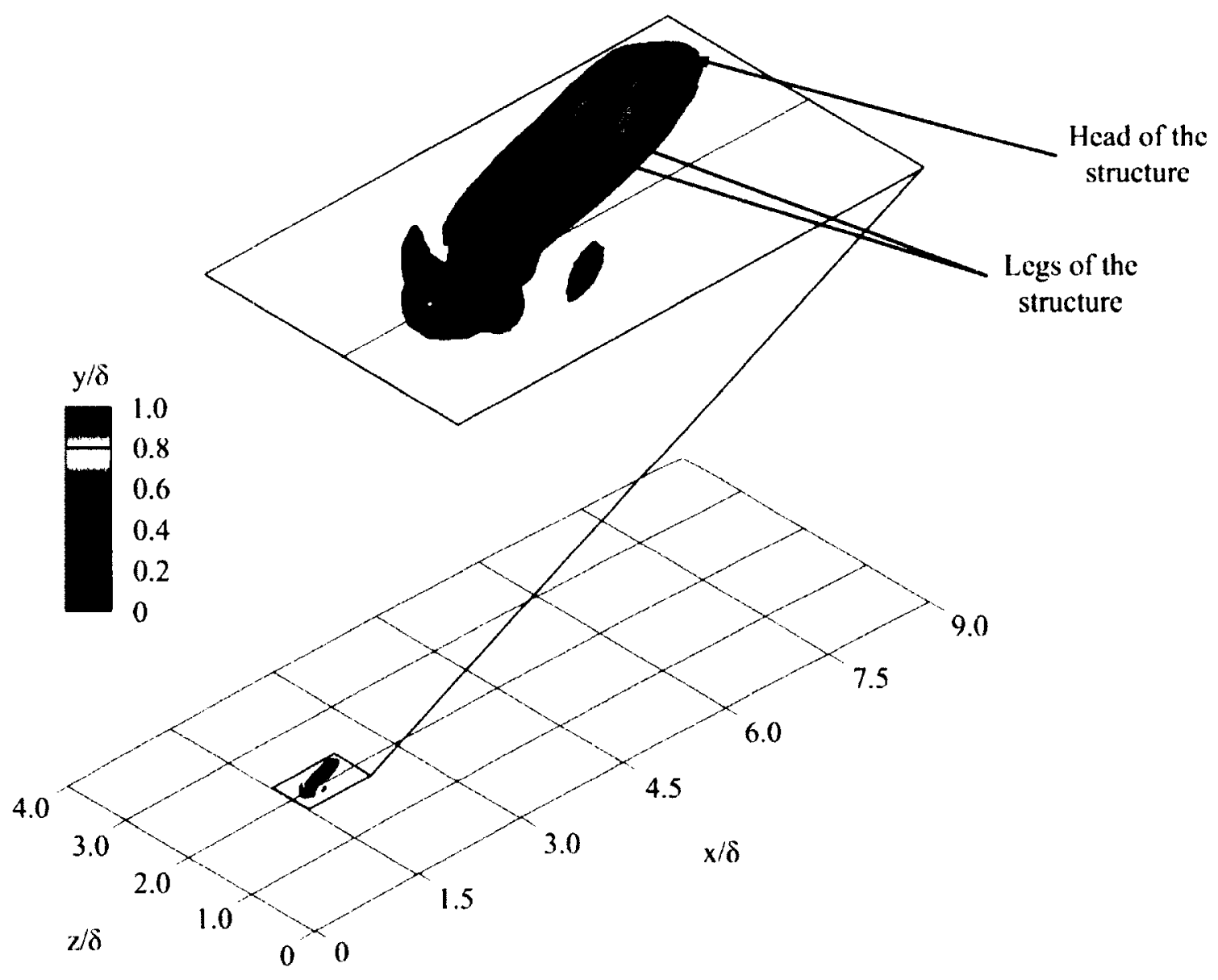

Figure 4.5: Stretching of the initial structure forming the head and legs of the vortex at $t^{+}=\mathbf{2 5}$. 

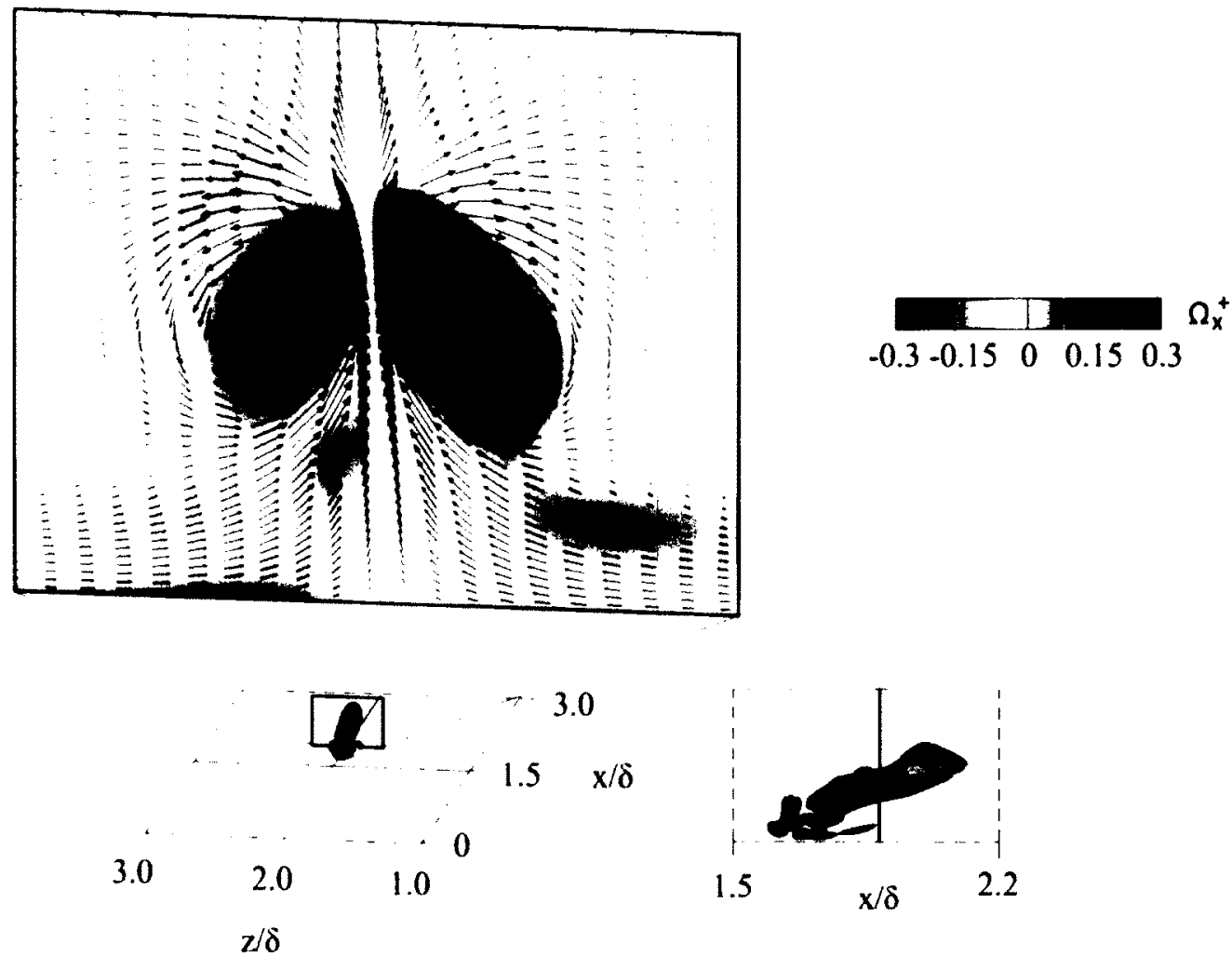

Figure 4.6: Fluid motion induced by the counter-rotating legs shown with the perturbation velocity vector in a spanwise-wall-normal plane at $t^{+}=25$. The full domain is not shown for clarity.
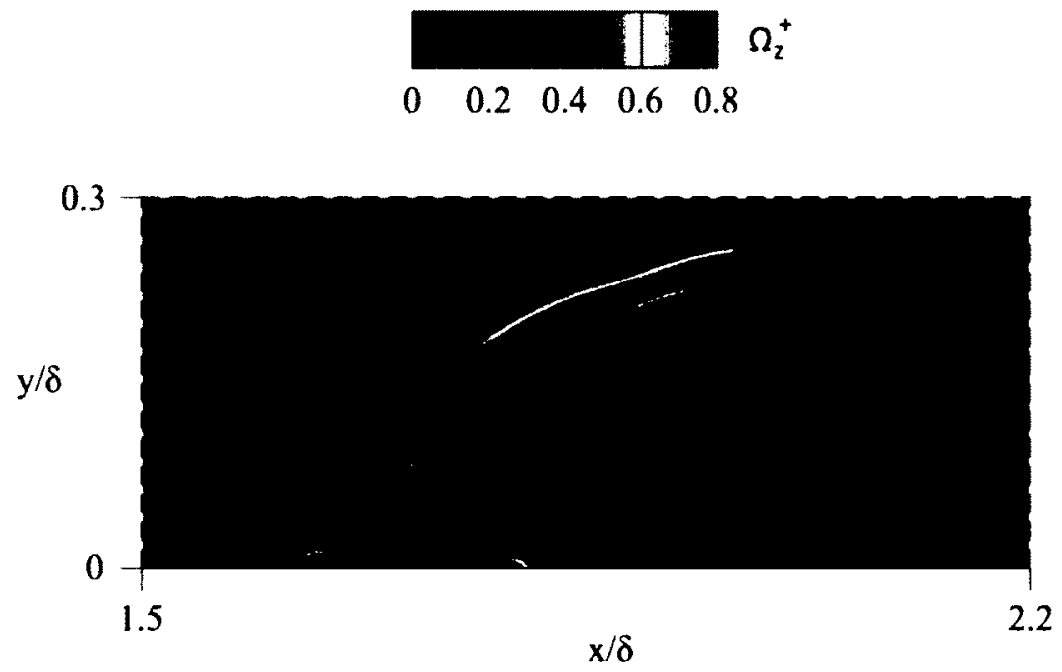

Figure 4.7: Formation of shear layer with spanwise oriented vorticity above the hairpin vortex at $t^{+}=25$. The full domain is not shown for clarity. 


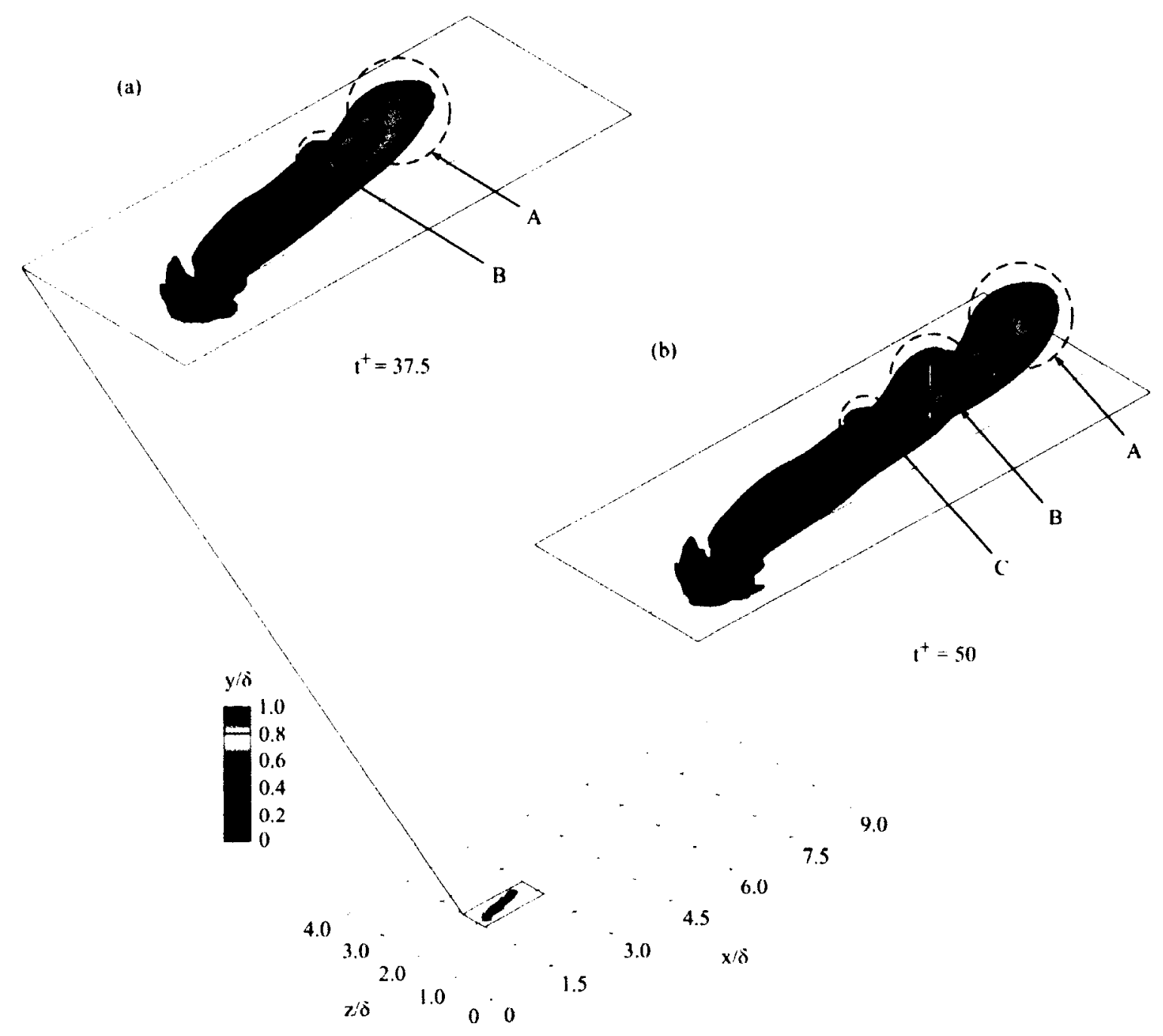

Figure 4.8: Evolution of the disturbance: (a) formation of the secondary structure $B$; (b) formation of the tertiary structure $C$.

As the hairpin vortices convect in the downstream direction, the counter-rotating quasi-streamwise vortices or the legs of the hairpin induce an upward velocity on each other shifting them progressively into higher momentum regions towards the freestream (Zhou et al., 1999; Adrian et al., 2000). This lift-up of the hairpin legs along with the induced sweep motions on the outboard side of the legs creates a region of streamwise vorticity close to the wall such that the orientation of the near-wall streamwise vorticity is opposite to that of the corresponding hairpin legs (Brooke and Hanratty, 1993). This 
streamwise vorticity layer seemingly attached to the wall is discernible in Figure 4.6 on each side of the legs of the hairpin. As the streamwise vorticity in the near-wall region is enhanced due to the mean shear and the induced spanwise flow of the legs, a new pair of streamwise vortices begins to roll-up consisting of vorticity that is of the opposite sign compared to each of the corresponding hairpin legs (see Figure 4.9). The evolution of opposite-signed streamwise vortices corresponding to each of the hairpin legs has been observed by several researchers including Acarlar and Smith (1987b), Bernard et al. (1993), Brooke and Hanratty (1993) and Zhou et al. (1999).
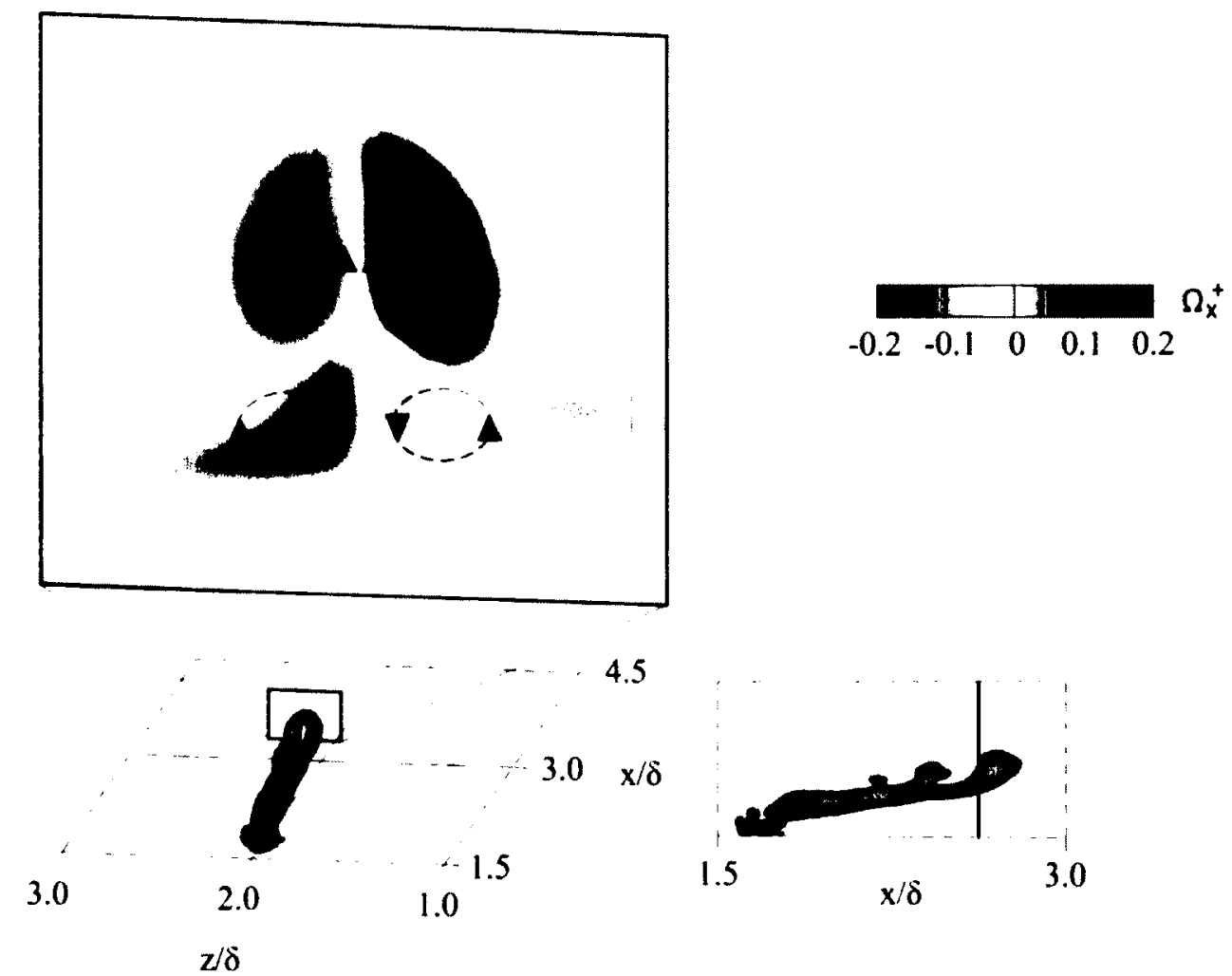

Figure 4.9: Formation of streamwise vortices consisting of vorticity of the opposite sign corresponding to each of the hairpin legs at $t^{+}=50$. The arrows indicate the fluid motion induced by the vortices. The streamwise vorticity contour level is selected to elucidate the newly forming streamwise vortices. 
Figure 4.9 suggests that the induced motion by the hairpin legs causes the newly formed streamwise vortices to move towards each other. Thus, it can be expected that as the legs of the hairpin move away from the wall due to mutual-induced effects, the new streamwise vortex pair will tend to move further apart. The streamwise vortex adjacent to the left leg of hairpin A is shown in Figure 4.10 and labelled as D while the other streamwise vortex of the pair adjacent to the right leg of hairpin A is not visible due to the orientation of the figure. Two new vortices labelled $\mathrm{E}$ and $\mathrm{F}$ develop at this stage as shown in Figure 4.10 at $t^{+}=75$, where structure E straddles the left leg of hairpin A and structure $\mathrm{F}$ forms on the downstream side of the head of hairpin $\mathrm{B}$. The formation of structures similar to $\mathrm{E}$ and $\mathrm{F}$ has not been reported in experiments or direct numerical simulations of turbulent boundary layers. The structure $E$ is possibly formed by the induction of low-speed fluid by the streamwise vortex $\mathrm{D}$ into the high-speed region on its outboard side leading to a shear layer instability with an instantaneous inflectional velocity profile, that causes the roll-up of structure $\mathrm{E}$ as shown in Figure 4.11. However, an interaction with the mean shear causes most of the spanwise vorticity of $E$ to be reoriented in the streamwise direction as visible in Figure 4.10 at $t^{+}=100$, where structure E connects to the lifted-up streamwise vortex D and straddles the left leg of hairpin A. It should be noted that a similar mechanism occurs on the right side of the vortex train but is not shown in Figure 4.10. The vortex $\mathrm{F}$ seems to be emanating from an interaction between the low-speed fluid ejected upwards between the legs of hairpin A and the highspeed fluid swept downwards by the head of hairpin B, which causes an unstable shear layer to form between the ejection and sweep motions, causing a roll-up of vortex $F$. 


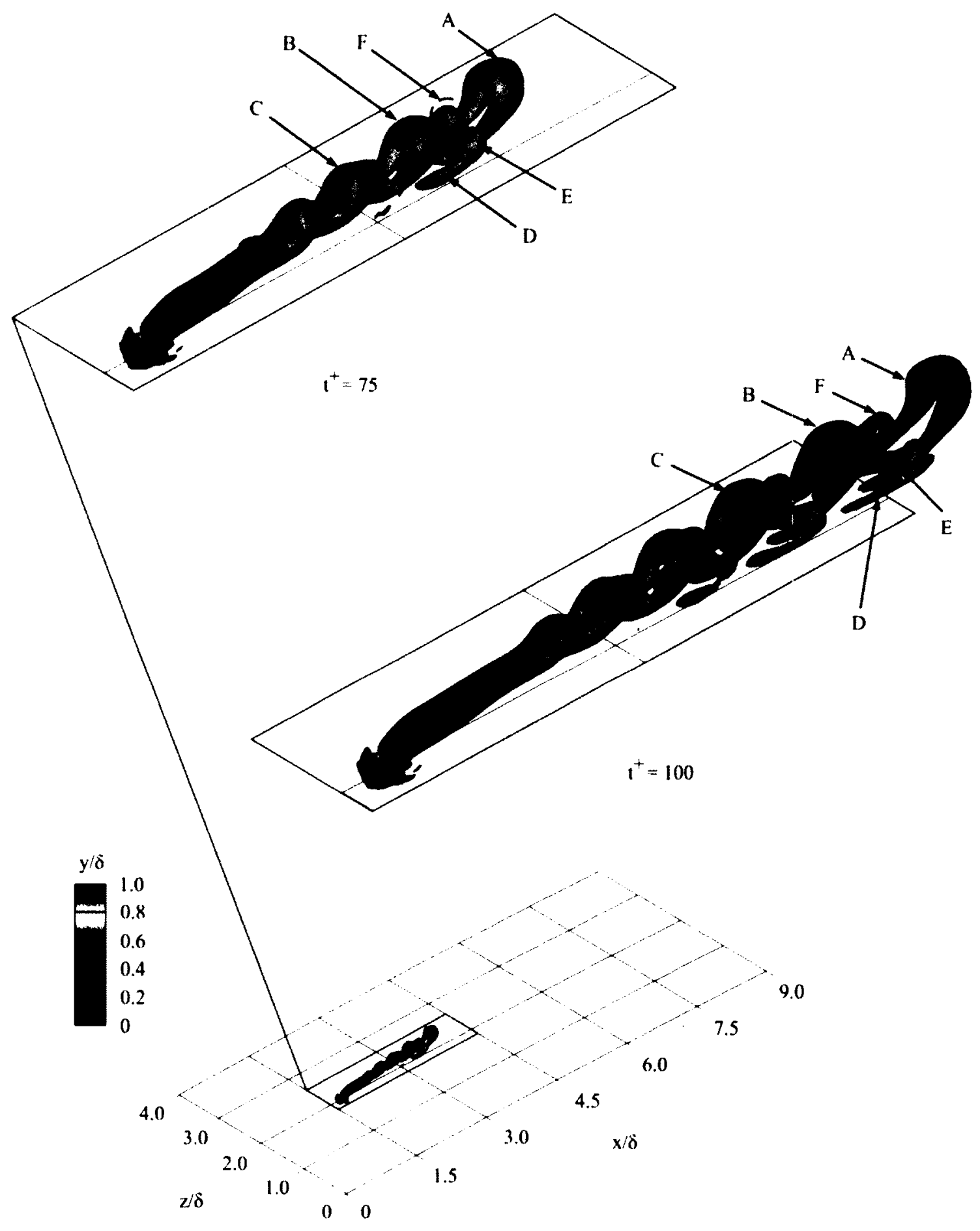

Figure 4.10: Evolution of the disturbance at $t^{+}=75$ and $t^{+}=100$. 


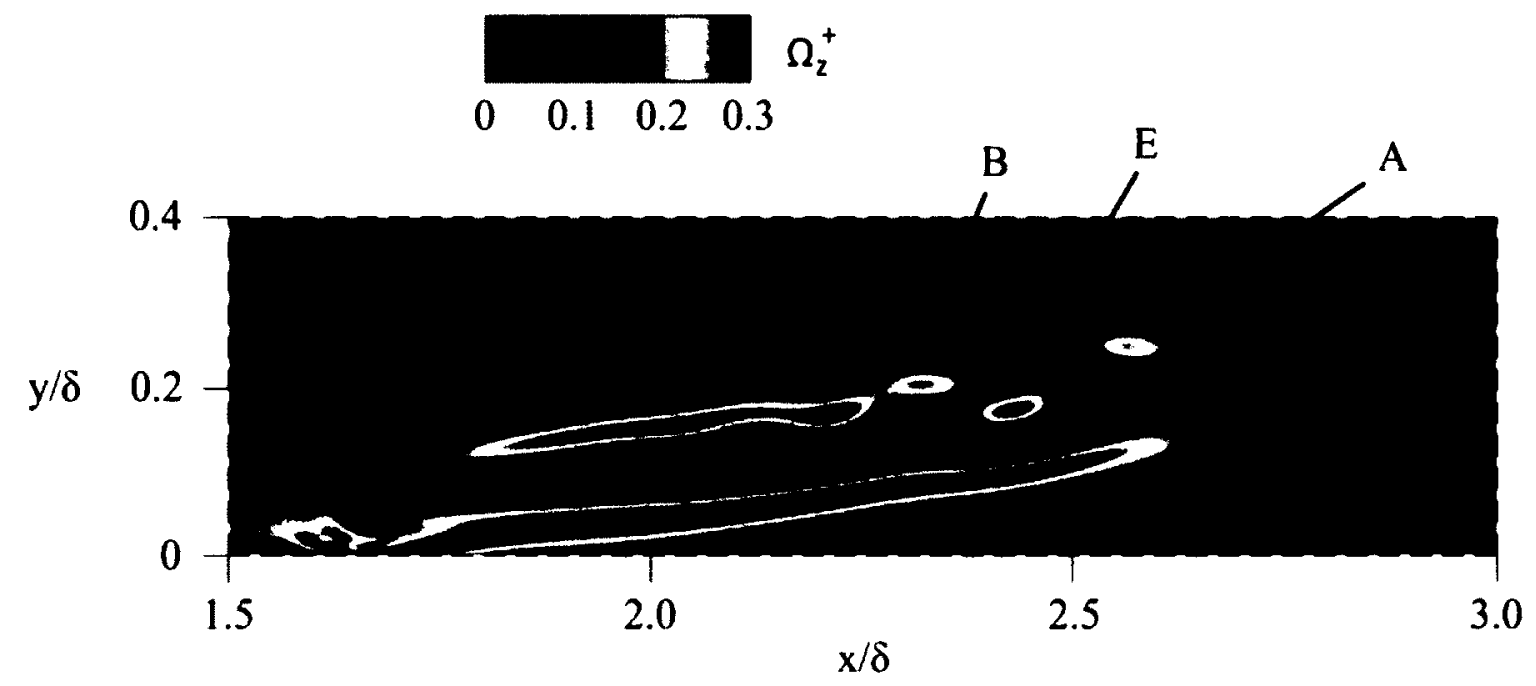

Figure 4.11: Shear layer with spanwise oriented vorticity at $t^{+}=62.5$ that rolls up to form structure $\mathbf{E}$.

Figure 4.12 shows the vortical structures at $t^{+}=125$ that correspond to the condition of the disturbance $\Delta t^{+}=25$ units after the momentum source is deactivated. One observation pertaining to Figure 4.12 is the dissipation of structures similar to $E$ and $F$ which can be described as follows: as the structures in the vortex train progressively move into higher-speed regions while convecting downstream, the oldest hairpin convects faster than the second oldest one due to its higher wall-normal extent, and so on. Thus, hairpin A convects faster than hairpin B and moves away from the vortices $\mathrm{E}$ and F. Hairpin B stabilizes the flow downstream of its head and on the outboard sides of its legs by sweep motions, and thus exerts a stabilizing influence on structures $\mathrm{E}$ and $\mathrm{F}$ causing them to be dissipated. Another interesting feature of the structures that can be observed in Figure 4.12 is the change in inclination of the hairpin heads from the newest hairpin to the oldest one. This can be explained based on the observation that as the hairpins convect in the downstream direction, the legs induce a wall-normal velocity on the head away from the wall causing the head to rise into the higher-speed region. The 
mean shear decreases away from the wall and hence the legs are stretched more than the head causing elongation of the legs and strengthening the vorticity present in the legs more than that of the head. The legs now induce an even stronger wall-normal motion on the head causing the head to rise even further in the boundary layer. This results in a reorientation of the quasi-streamwise vorticity in the portion connecting the head and legs of the hairpin to quasi-wall-normal vorticity tilting the head to an almost vertical orientation with respect to the wall. Acarlar and Smith (1987b) offer a slightly different explanation that as the head moves further towards the freestream, the mean shear decreases resulting in a reduced stretching which allows the head to incline in a near vertical orientation through a backward self-induced motion. It is quite plausible that both of these proposed mechanisms contribute to the shaping of the hairpin vortices as observed.

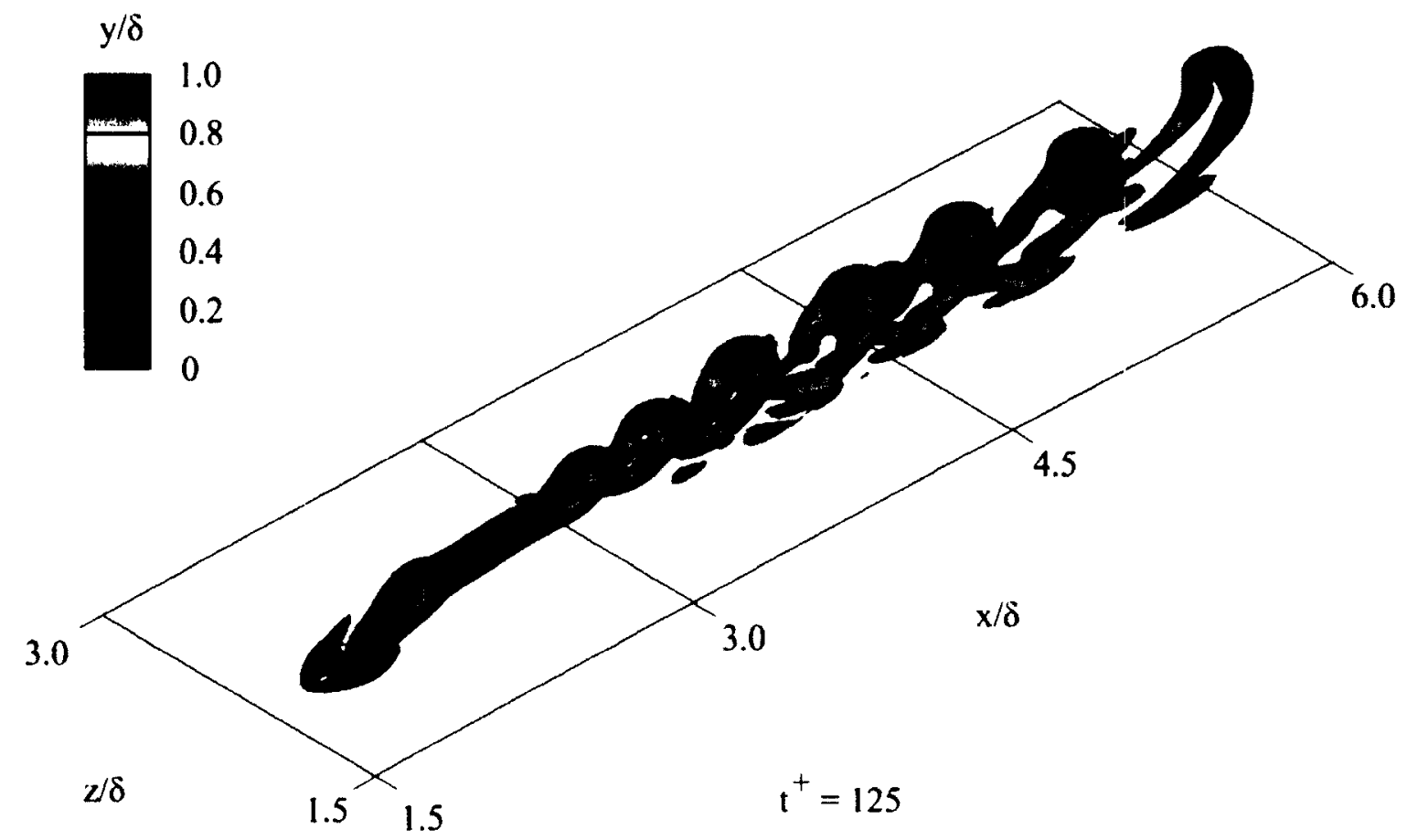

Figure 4.12: Hairpin-like vortical structures at $t^{+}=125$ with the flow disturbance deactivated at $t^{+}=100$. The full domain is not shown for clarity. 
Figure 4.13 shows the hairpin vortices at $t^{+}=200$ indicating that deactivating the momentum-source disturbance causes the hairpins that were formed as a result of the disturbance to get weaker and slowly dissipate as they rise in the boundary layer. New vortices are beginning to develop as perceived from the upstream end of the vortex train. The effect of introducing the disturbance in the laminar flow-field is thus to trigger transition and expedite the transitional phase to turbulence.

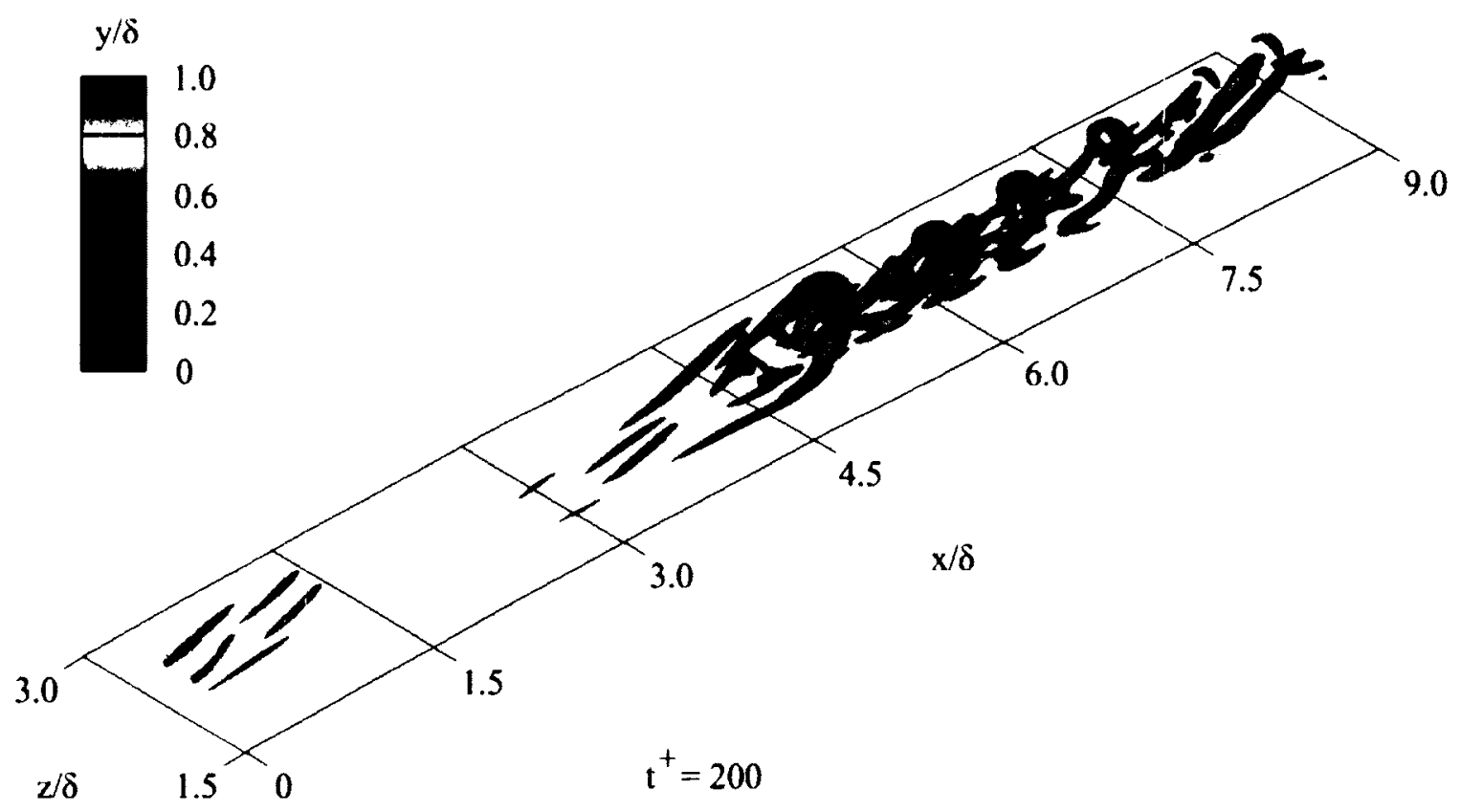

Figure 4.13: Hairpin vortices at $t^{+}=200$ showing the dissipating vortices that were formed while the disturbance was active. The full domain is not shown for clarity.

\subsubsection{Comparison of Evolution of the Initial Disturbance on the Mildly-Curved Streamwise Convex Surface and the Flat Surface}

The flat-surface simulation is conducted in exactly the same manner as the convex-surface simulation. For the curved-surface case, a radial pressure gradient exists which acts to stabilize the flow. Thus, the development of vortical structures on the 
convex-surface case is under the influence of a stabilizing radial pressure gradient. However, the growth of the disturbance is observed to be similar for the flat-surface and convex-surface cases until the source is deactivated implying that the radial pressure gradient does not strongly influence the initial development of vortical structures in this case. This can be observed in Figure 4.14 and Figure 4.15 at $t^{+}=25$ and 125, respectively.

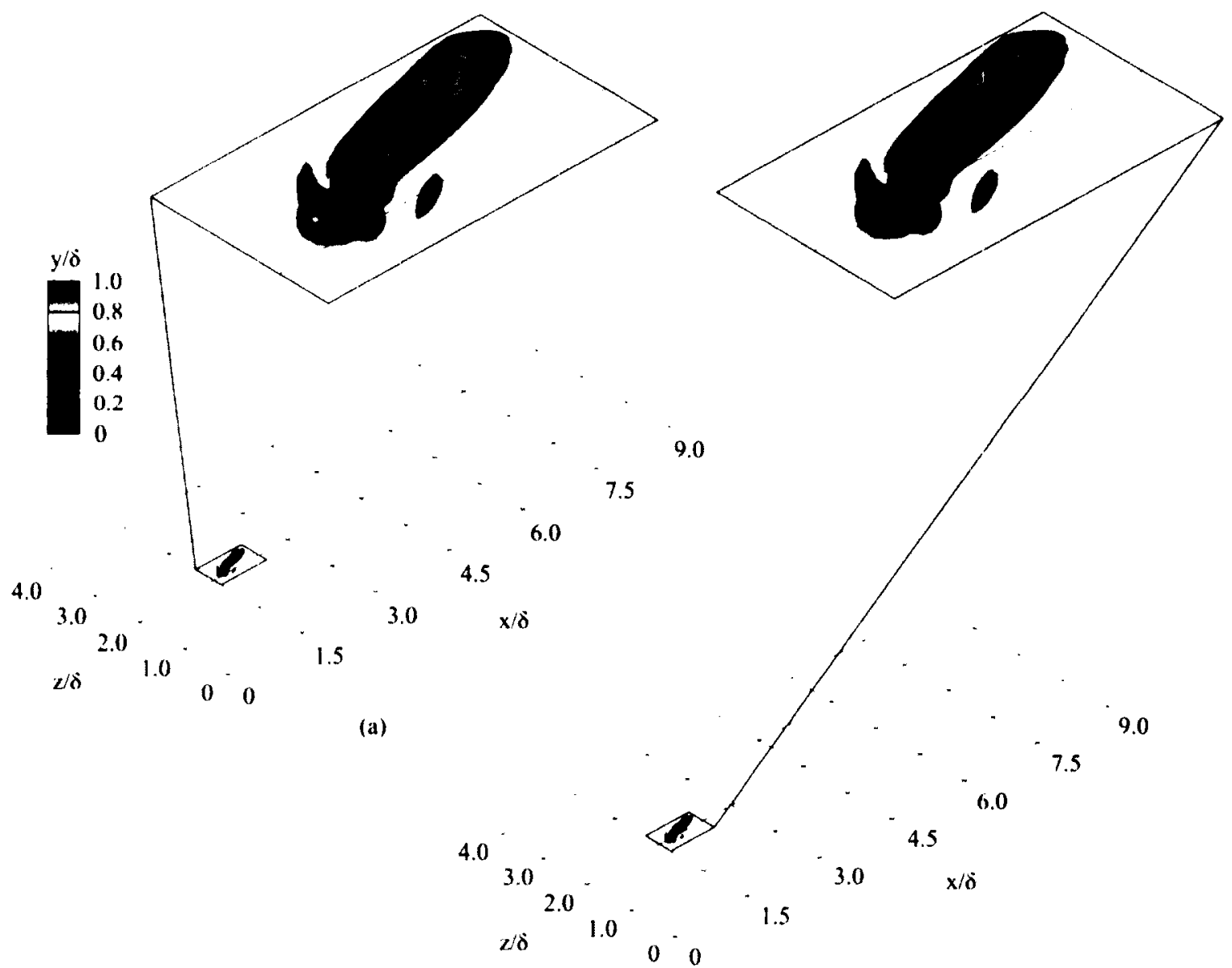

(b)

Figure 4.14: Evolution of disturbance compared at $t^{+}=25$ : (a) flat surface (Case 1); (b) convex surface (Case 2). 


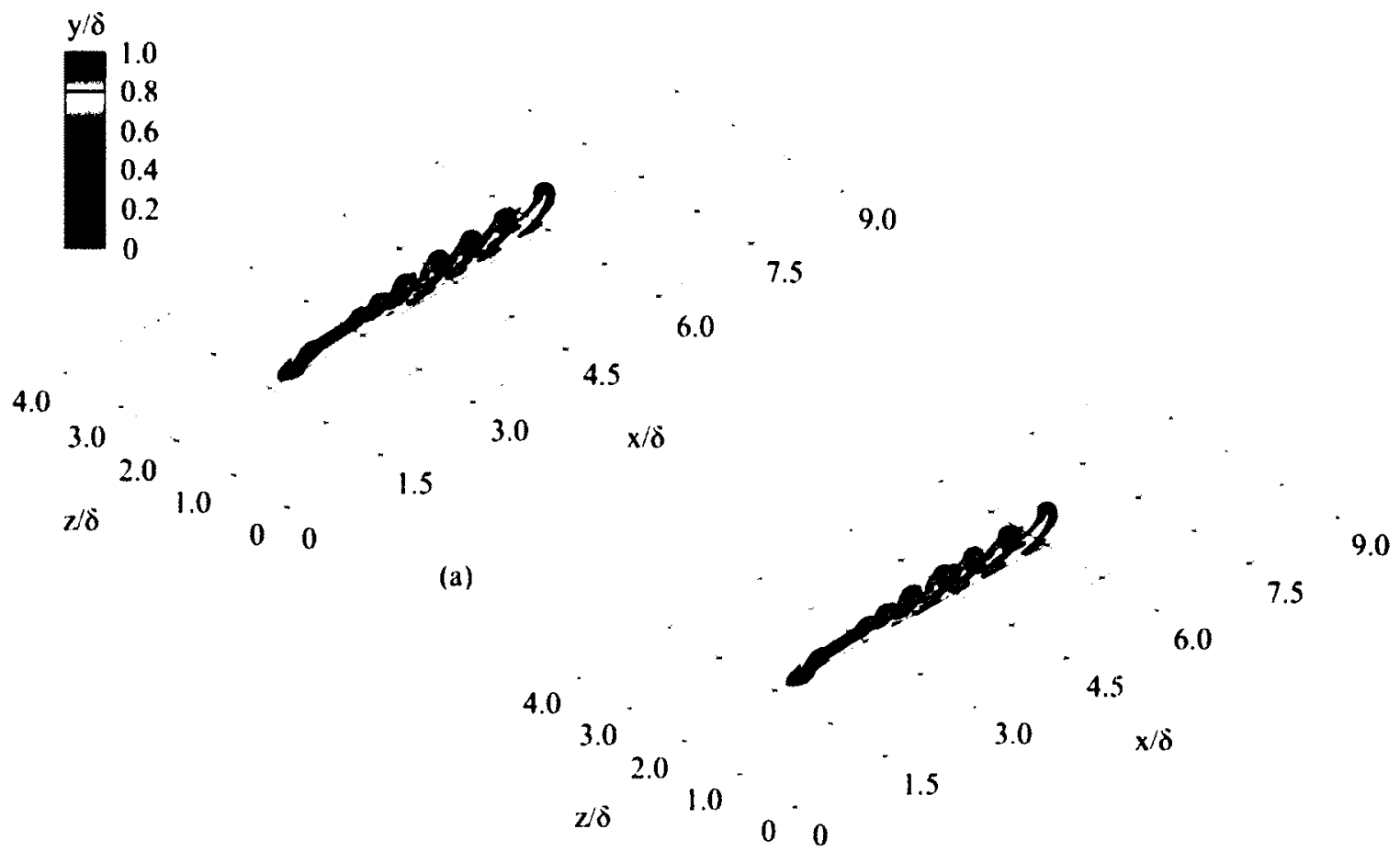

(b)

Figure 4.15: Evolution of disturbance compared at $t^{+}=125$ : (a) flat surface (Case 1); (b) convex surface (Case 2).

The difference between the two cases begins to appear at $t^{+}=250$ as seen in Figure 4.16 where the region in and around the area labelled I in the flat-surface case (Case 1) shows signs of increased turbulence activity containing more vortical structures than in the convex-surface case (Case 2). The increased turbulence activity within the boundary layer in the flat-surface case is also reflected in Figure 4.17 and Figure 4.18 as multiples of hairpin-like vortical flow structures develop in the boundary layer. The formation of multiples of hairpin-like vortical structures is often a result of localized inviscid instabilities created by older hairpin vortices due to induced wall-normal motions (McAuliffe and Yaras, 2007). The reduced turbulence activity in the convex-surface case is likely the result of the stabilizing influence of streamwise convex surface curvature that causes a radial equilibrium to exist between the wall-normal pressure gradient and the 
centrifugal force, and the presence of such a radial equilibrium mechanism tends to reduce the wall-normal motions in the boundary layer. Also, since the centrifugal force is proportional to the square of the streamwise velocity component, the magnitude of the centrifugal force and thus the radial pressure gradient increases with distance from the wall (So and Mellor, 1973). Thus, the stabilizing influence of convex surface curvature tends to increase with wall-normal distance leading to the suppression of the hairpin vortices convecting into higher speed regions. This is visible in Figure 4.18 based on the illustration of the vortical structures according to wall-normal height, indicating that some of the large-scale structures in the flat-surface case are at the edge of the boundary layer while similar structures in the convex-surface case are below $0.8 \delta$.

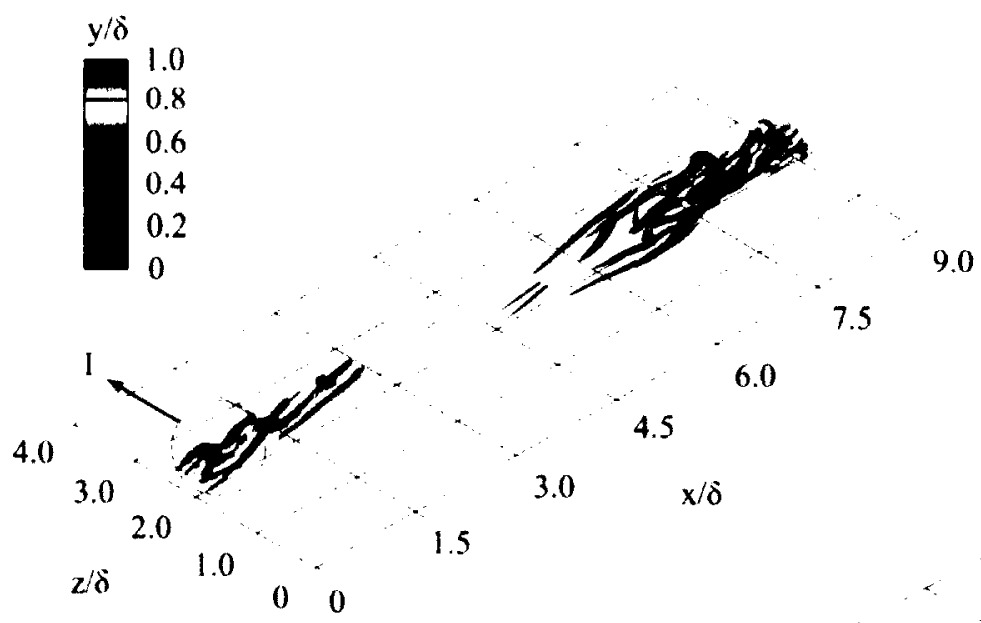

(a)
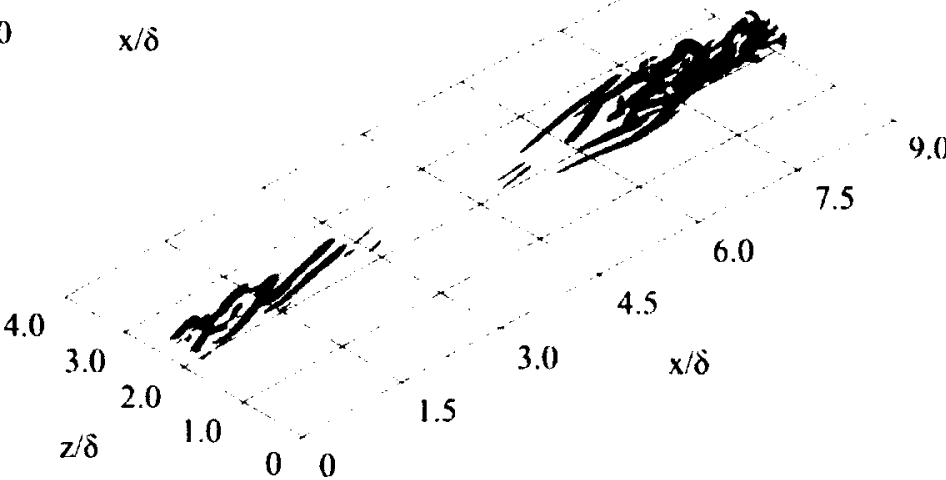

(b)

Figure 4.16: Evolution of disturbance compared at $t^{+}=250$ : (a) flat surface (Case 1); (b) convex surface (Case 2). 


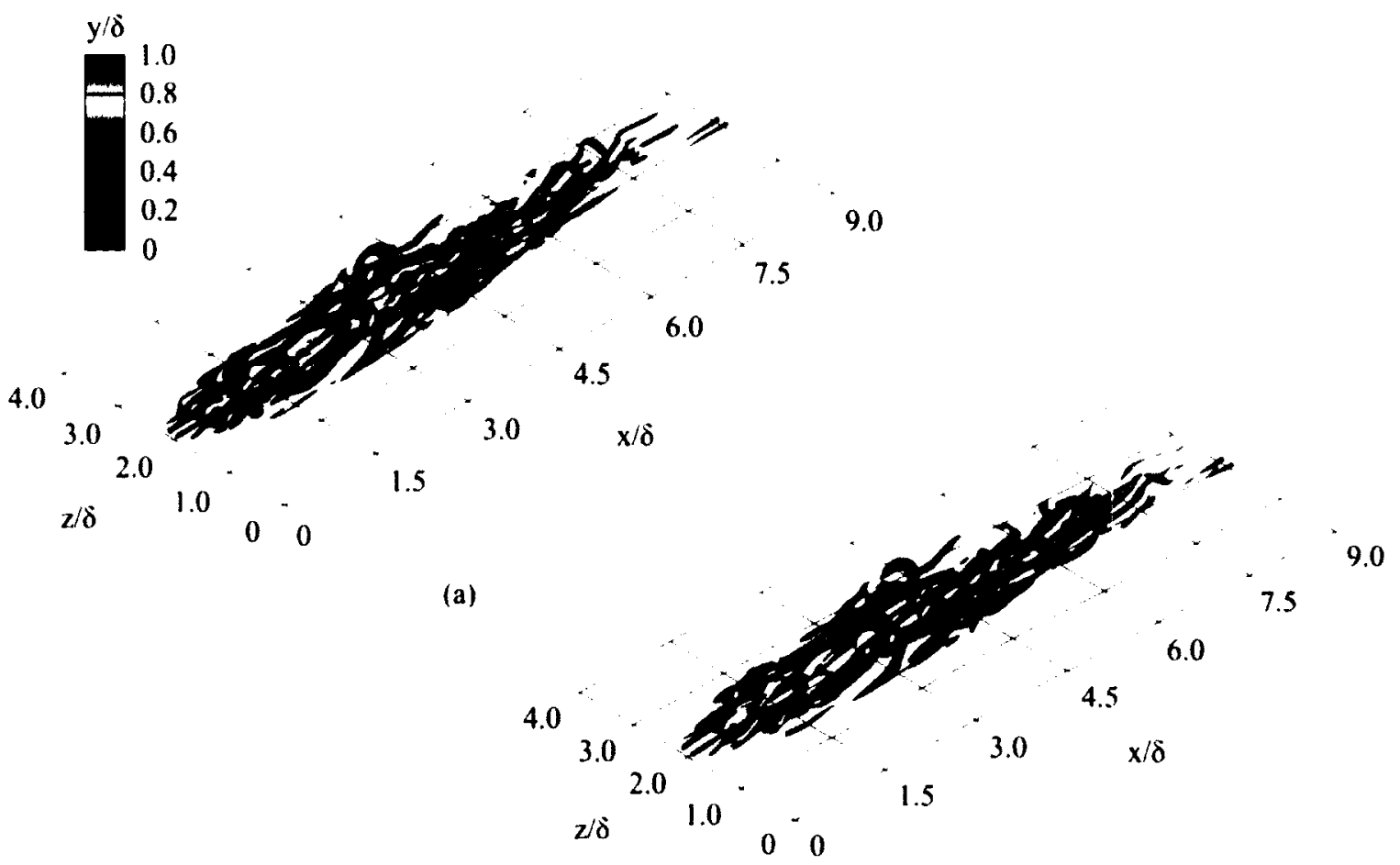

(b)

Figure 4.17: Evolution of disturbance compared at $t^{+}=400$ : (a) flat surface (Case 1); (b) convex surface (Case 2 ). 


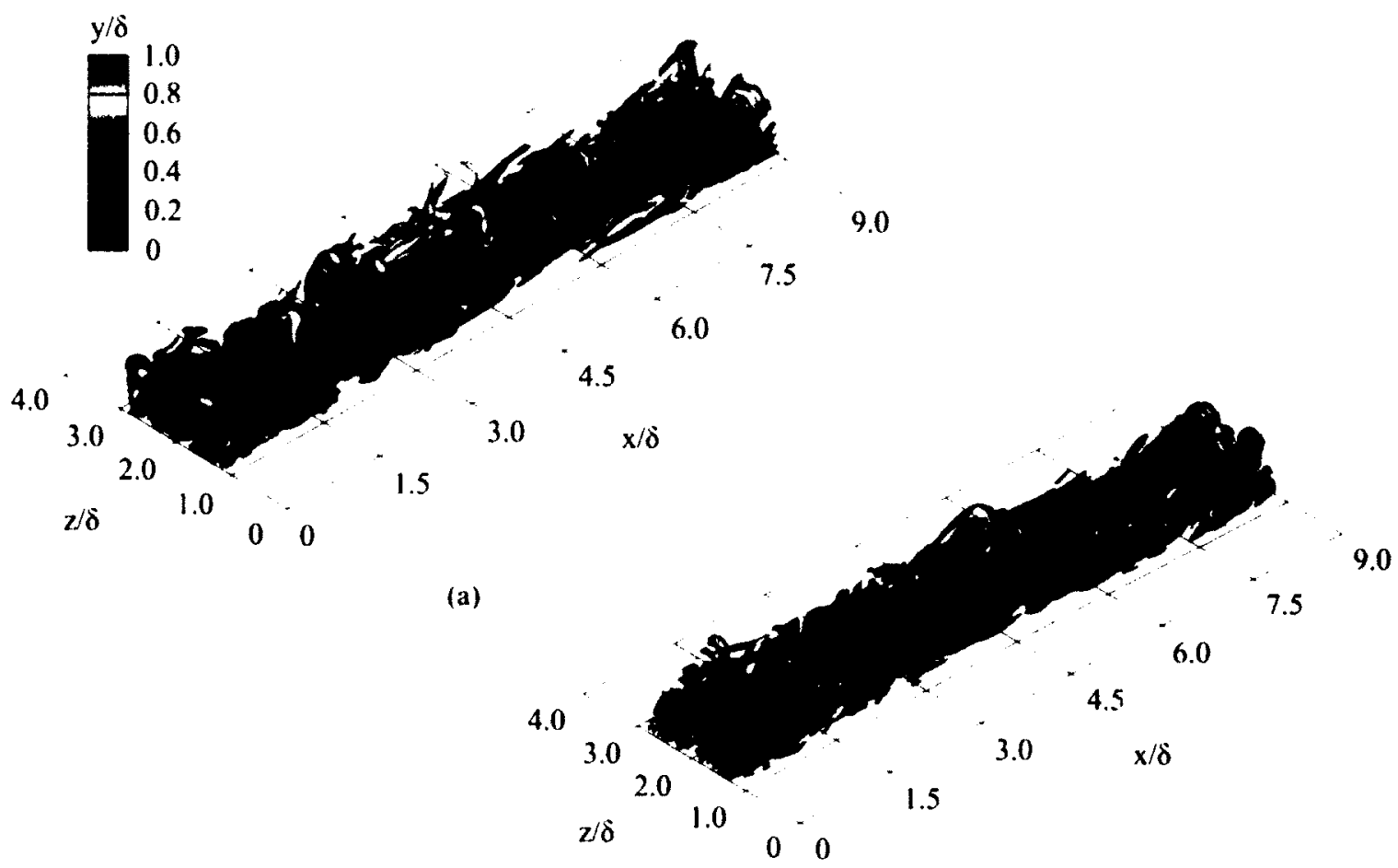

(b)

Figure 4.18: Evolution of disturbance compared at $t^{+}=600$ : (a) flat surface (Case 1); (b) convex surface (Case 2).

4.3 Effect of Streamwise Convex Surface Curvature on Turbulence in the Boundary Layer

Once the boundary layer develops to a fully turbulent state on both the flat and convex surfaces, the representation of hairpin vertices through iso-surfaces of the second invariant of the velocity gradient tensor $(Q)$ is unable to provide substantial understanding of the effect of convex surface curvature on turbulence. This can be observed from Figure 4.19 where the boundary-layer turbulence activity appears to be similar in the two cases although differences in the development of the vortical structures in the boundary layer were observed during the transitional regime. In order to

86 
characterize the effect of streamwise convex surface curvature on turbulence, it would be appropriate to first study the boundary-layer mean-flow properties and then probe the behaviour of the hairpin vortices in more detail.

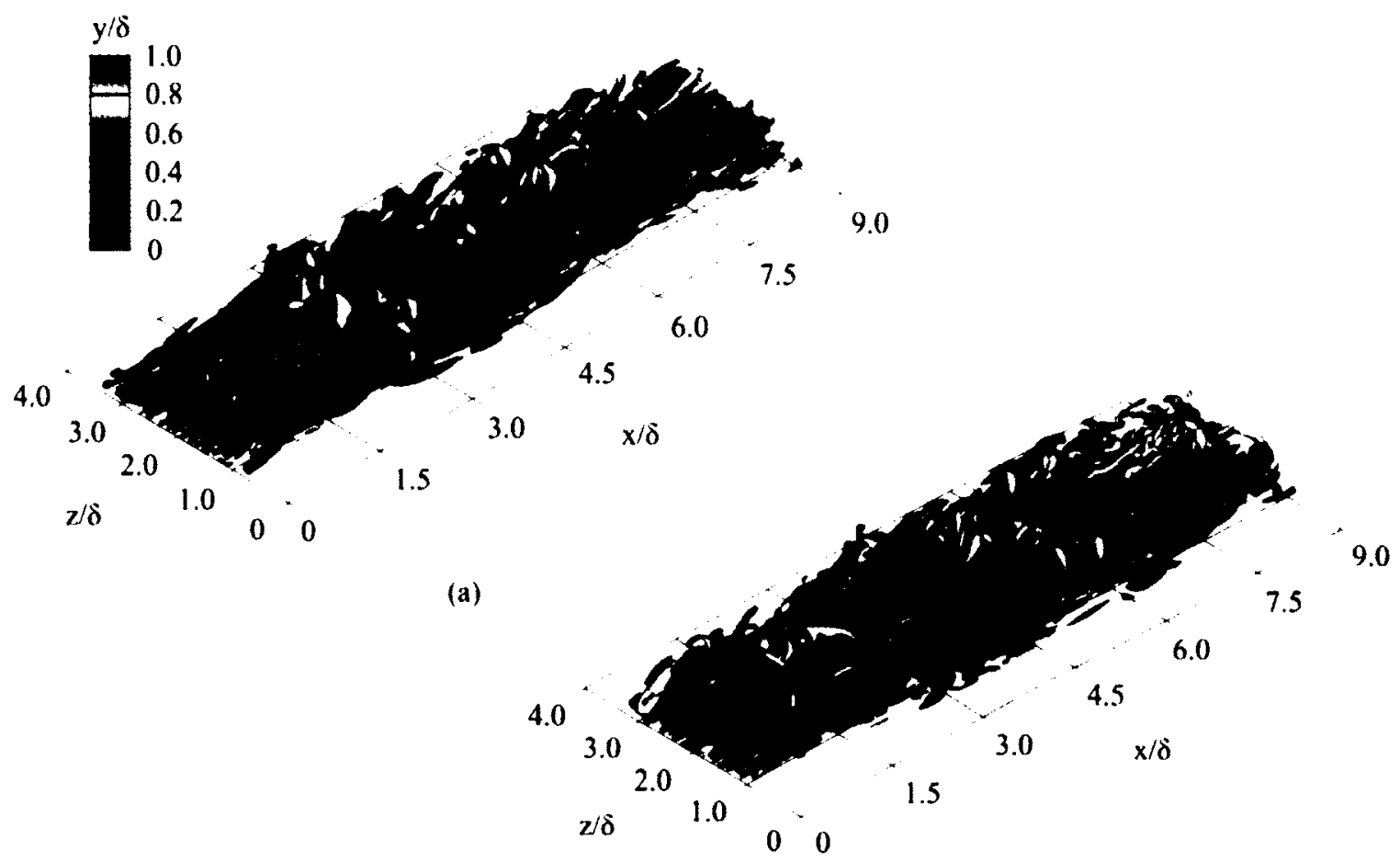

(b)

Figure 4.19: Iso-surfaces of $Q^{+}=0.0027$ showing hairpin-like structures in a fully turbulent boundary layer for (a) flat surface (Case 1); (b) convex surface (Case 2).

The mean velocity profiles for Case 1 and Case 2 are observed to overlap near the wall $\left(y^{+}<50\right)$ as seen in Figure 4.20 and deviate from each other by an amount less than $5 \%$ of the boundary-layer edge velocity away from the wall. The velocity magnitudes are slightly larger for Case 2 in the outer part of the boundary layer, thus yielding a slightly less full profile compared to Case 1. This result is suggestive of reduced turbulence activity in Case 2, for cross-stream momentum exchange driven by turbulence activity is responsible for the relatively high momentum in the inner region of a turbulent boundary 
layer compared to its laminar counterpart. Some effect of the stabilizing influence of streamwise convex surface curvature is observed on the rms of velocity fluctuations for Cases 1 and 2 (see Figure 4.21), especially in the streamwise and wall-normal velocity components. This is expected as the radial equilibrium between the wall-normal pressure gradient and the centrifugal force tends to oppose the wall-normal motions, causing a reduction in the wall-normal rms velocity fluctuations. The suppression of wall-normal motions also leads to a reduction in the wall-normal transfer of fluid particles with varying streamwise velocity from their respective positions in the boundary layer, causing a decrease in the streamwise rms velocity fluctuations.

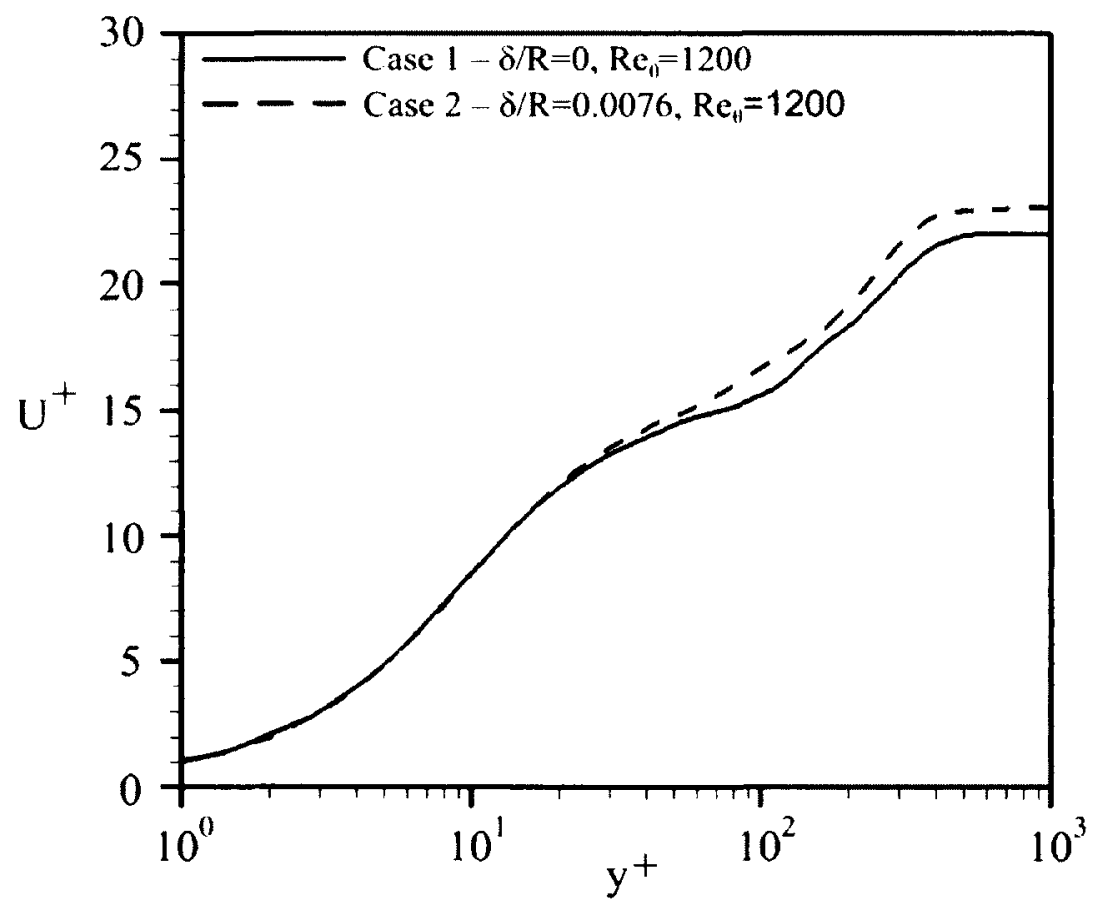

Figure 4.20: Comparison of mean velocity profiles for flat-surface (Case 1) and convex-surface (Case 2). 


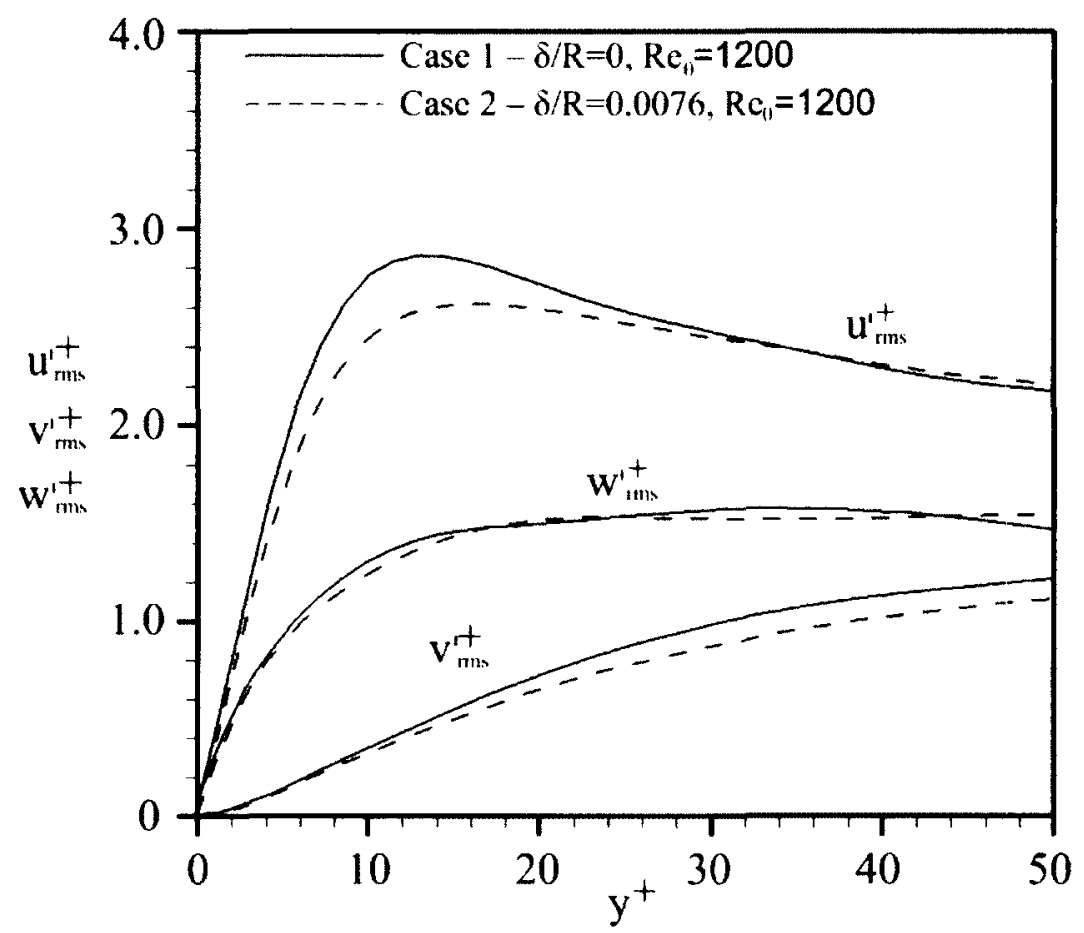

Figure 4.21: Comparison of fluctuating velocity fields for flat-surface (Case 1) and convex-surface (Case 2).

In order to visualize the hairpin vortices in greater detail, it would serve useful to study these flow structures in the context of a wave packet formation. The heads of the hairpin vortices can be visualized through contours of spanwise vorticity in a streamwisewall-normal plane as depicted in Figure 4.22. The wave packets shown in the figure are representative of multiple wave packets that were studied in the flow field of Cases 1 and 2. As noted in Figure 4.22, the wave packets contain three to four streamwise-aligned hairpin vortices in both cases. Nine wave packets were identified in the computed flow fields for detailed analysis, and their analysis reveals that the streamwise spacing of the heads of the hairpin vortices in the near-wall region $\left(y^{+}<120\right)$ is in the range of $100-140$ wall units for Cases 1 and 2, which is consistent with published literature (Zhou et al., 1999; Adrian et al., 2000). However, as the hairpins grow in wall-normal height due to 
mutual- and self-induction (Zhou et al., 1999; Adrian et al., 2000; Brinkerhoff and Yaras, 2011), they extend into regions of faster moving fluid and hence they are stretched in the streamwise direction through an interaction with the mean shear. The streamwise spacing of the hairpin vortices when the wave packets have convected further away from the wall is depicted in Figure 4.23 and is observed to be in the range of 150-200 wall units for Case 1, which is again consistent with published literature. For Case 2, a reduced streamwise spacing is observed and is in the range of 100-150 wall units. McAuliffe and Yaras (2007) suggest that the creation of multiple loops in a wave packet is the result of a sequence of localized inviscid instabilities initiated by the primary (initial) vortex loop in the wave packet. As illustrated in Figure 4.22, the strong similarity in the spacings of the hairpin vortices of a wave packet in the near-wall region for Cases 1 and 2 indicates that the difference in the fullness of the mean velocity profile between these cases as observed in Figure 4.20 does not strongly influence the wavelength of this inviscid-instability. However, it appears that as the vortices evolve further, the degree of streamwisestretching of the vortices differs between Cases 1 and 2. The stability-promoting effect of the radial equilibrium that exists between wall-normal pressure gradient and centrifugal force in the curved case scales on the square of the local streamwise velocity. As such, this stabilizing effect is expected to increase with distance from the wall. Development of difference between the structures of the wave packets for Cases 1 and 2 as they move away from the wall is therefore not surprising. 
(a)

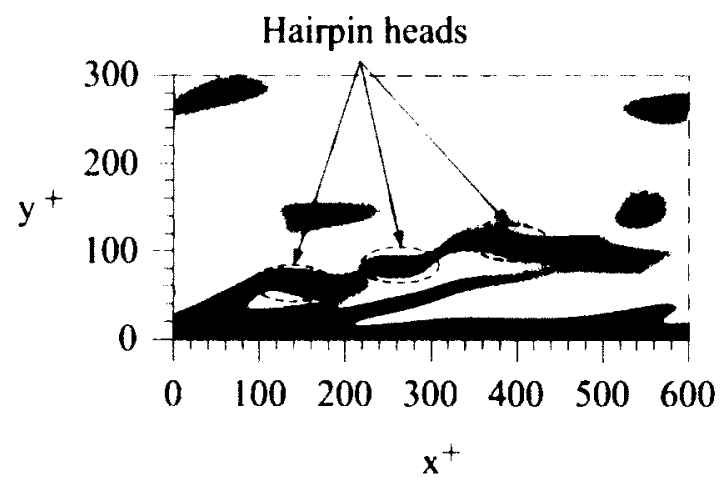

(b)

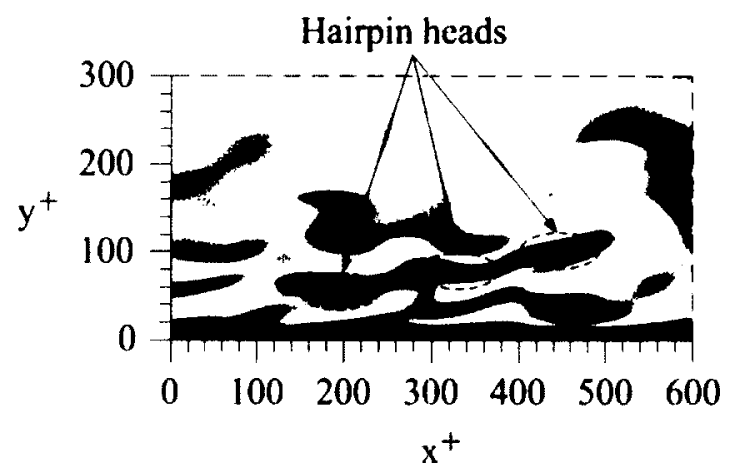

Figure 4.22: Streamwise spacing of hairpin vortices in a wave packet in the nearwall region for (a) Case 1; (b) Case 2 visualized through flood plots of spanwise component of vorticity.

(a)

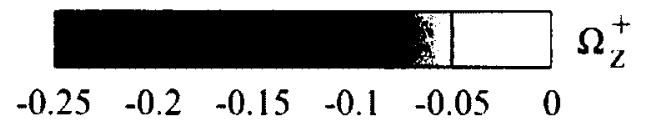

(b)

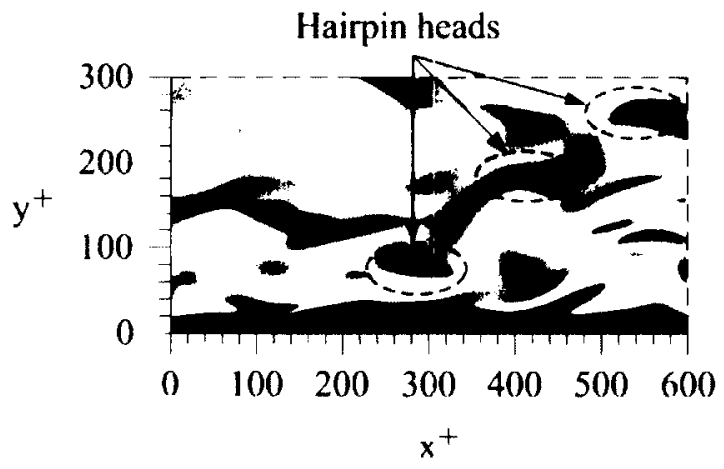

Figure 4.23: Streamwise spacing of hairpin vortices in a wave packet further away from the wall for (a) Case 1; (b) Case 2 visualized through flood plots of spanwise component of vorticity. 
Spanwise spacing of the hairpin vortices is determined by the balance of viscous dissipation and the kinetic energy of wall-normal motions (Chernyshenko and Baig, 2005). The induced upward motion between the legs of a hairpin vortex and the downward motion outside these legs result in spanwise-alternating high- and low-speed streaks in the instantaneous local streamwise velocity field, with the resultant shear layers between these streaks providing viscous energy dissipation and promoting inviscid local instabilities that contribute to the sequential development of hairpin vortices in a wave packet, as noted above (Zhou et al., 1999; McAuliffe and Yaras, 2007; Brinkerhoff and Yaras, 2011). In this context, for a given boundary layer, an optimum spanwise spacing of hairpin vortices and hence streaks exists that maximizes the strength of the hairpin vortices. In a zero-pressure-gradient turbulent boundary layer developing on a flat surface, this optimum spacing corresponds to $\Delta z^{+} \approx 100$ (Butler and Farrell, 1993; Zhou et al., 1999; Chernyshenko and Baig, 2005). The average value of the streak spacings for Case 1 visualized through the instantaneous fluctuation distribution of the streamwise velocity component in Figure 4.24(a) is consistent with this value. For Case 2, the average streak spacing is noted to be slightly larger (Figure 4.24(b)), which is consistent with the fact that the radial-equilibrium mechanism promoting stability in the case of convex-surface curvature would tend to work against the wall-normal motions induced by the hairpin vortices. 


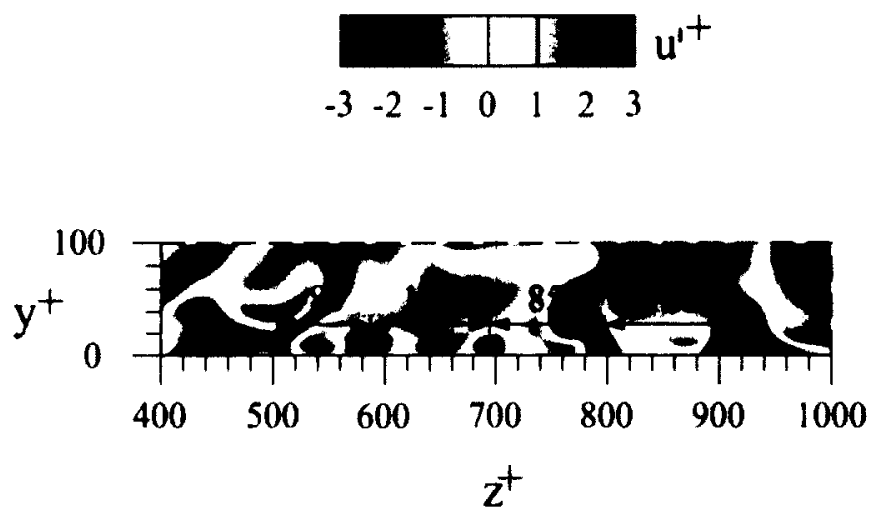

(a)

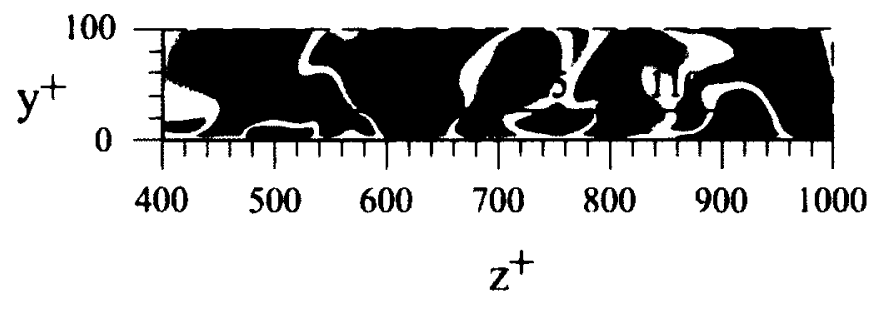

(b)

Figure 4.24: Spanwise streak spacing observed in the distribution of streamwise component of fluctuation velocity for (a) Case 1; (b) Case 2.

\subsection{Effect of Streamwise Convex Surface Curvature on Forced Convection Heat Transfer}

In the heated test cases (Cases 3 and 4), water at supercritical thermodynamic state is heated in a thermodynamic region of state with strong temperature dependence of the fluid properties which occurs within a narrow temperature band centered on the pseudo-critical point. The pseudo-critical point is defined by the temperature, $T_{p c}$, at which the specific heat is at its maximum value for a given pressure above the thermodynamic critical pressure. A recent DNS study by Azih et al. (2012) demonstrated that the coherent flow structures of turbulence and the resultant convection heat transfer 
rate in a flat-surface boundary layer are affected by such significant fluid-property variations with temperature. In the present study, the surface heat-flux value is selected to correspond to the simulation of Azih et al. (2012) in which improved heat transfer was observed, and yields a mean surface-temperature variation from $T / T_{p c} \approx 0.97$ to $T / T_{p c} \approx 1.02$ over the streamwise length of interest (see $x / \delta$ in Table 4.1). The relevant streamwise length, $x$, for the present computations is the product of the boundary-layer edge velocity and the duration over which the boundary layer was developed.

The Nusselt number values for the heated mild-curvature case (Case 3), the heated strong-curvature case (Case 4) and the study of Azih et al. (2012) are presented in Table 4.1 along with the streak spacings for all of the test cases. The Nusselt number is indicative of the heat transfer rate for a given temperature potential, and is defined as $N u=q_{w} x / k\left(T_{w}-T_{b}\right)$, where $q_{w}$ is the wall heat flux, $x$ is the streamwise length of the heated surface, $T_{w}$ and $T_{b}$ are the wall- and freestream-fluid temperatures, respectively, and $k$ is the thermal conductivity at the film temperature, defined as $T_{f}=\left(T_{w}+T_{b}\right) / 2$. To facilitate comparison with the case of Azih et al. (2012), the predicted Nusselt number for all of the test cases is obtained though the correlation proposed by Churchill (1976) presented in Eqns. 4.4 and 4.5. This correlation accounts for the laminar, transitional and turbulent flow regimes over the streamwise length of interest. 
Table 4.1: Nusselt number values and streak spacings.

\begin{tabular}{|c|c|c|c|c|c|c|c|}
\hline Test cases & $\delta / R$ & $\begin{array}{c}\boldsymbol{x} / \boldsymbol{\delta} \\
\left(\boldsymbol{x}_{\text {transition }} / \boldsymbol{\delta}\right)\end{array}$ & $\begin{array}{c}\text { Working } \\
\text { fluid }\end{array}$ & $\begin{array}{c}\boldsymbol{q} / \boldsymbol{G} \\
(\mathbf{k J} / \mathbf{k g})\end{array}$ & $\begin{array}{c}\text { Computed } \\
\text { Nusselt } \\
\text { number }\end{array}$ & $\begin{array}{c}\text { Predicted } \\
\text { Nusselt } \\
\text { number } \\
(\text { Eqn. 4.4) }\end{array}$ & $\begin{array}{c}\text { Streak } \\
\text { spacing } \\
\text { (wall } \\
\text { units) }\end{array}$ \\
\hline Case 1 & $\mathbf{0}$ & - & Air & 0 & - & - & 100 \\
\hline Case 2 & 0.0076 & - & Air & 0 & - & - & 110 \\
\hline Case 3 & 0.0017 & $\begin{array}{c}25.6 \\
(15.2)\end{array}$ & $\begin{array}{c}\text { Supercritical } \\
\text { Water }\end{array}$ & 0.9 & 500 & 412 & 95 \\
\hline Case 4 & 0.0400 & $\begin{array}{c}26.0 \\
(15.7)\end{array}$ & $\begin{array}{c}\text { Supercritical } \\
\text { Water }\end{array}$ & 0.9 & 472 & 417 & 140 \\
\hline $\begin{array}{c}\text { Azih et al. } \\
(2012) \\
\left(R e_{\mathbf{T}}=250\right)\end{array}$ & $\mathbf{0}$ & $\begin{array}{c}29.6 \\
(21.1)\end{array}$ & $\begin{array}{c}\text { Supercritical } \\
\text { Water }\end{array}$ & 0.9 & 533 & 431 & 72 \\
\hline
\end{tabular}

$$
\begin{gathered}
N u=0.4637\left[\varphi+\frac{\varphi^{8 / 5}}{209\left(1+\left(\frac{\varphi_{u}}{\varphi}\right)^{7 / 2}\right)^{2 / 5}}\right]^{1 / 2}+0.45 \\
\varphi=\frac{\operatorname{Re}_{x} \operatorname{Pr}^{2 / 3}}{\left(1+\left(\frac{0.02052}{P r}\right)^{2 / 3}\right)^{1 / 2}}
\end{gathered}
$$

Here, $\varphi_{u}$ is the value of $\varphi$ evaluated at the streamwise location where transition is completed as proposed by Churchill (1976). The streamwise distance at which transition occurs is listed in Table 4.1.

The computed Nusselt number values for Cases 3, 4 and that of Azih et al. (2012) clearly illustrate the reduction in the convection heat transfer coefficient - or increased thermal resistance - with increasing streamwise convex curvature of the surface. This trend is noted to be accompanied by increasing streak spacing with surface curvature (Table 4.1 and Figure 4.25). This implies a substantial role of reduced turbulence activity in the boundary layer under the stabilizing influence of convex-surface curvature in the observed trend in heat transfer. The predicted Nusselt number values are based on a 
correlation that does not account for the effects of surface curvature or the supercritical state of the working fluid. The small discrepancy in the predicted Nusselt number among the three cases is due to variations in the transition onset location and the difference in Reynolds number between the cases in the present study and the study of Azih et al. (2012). Comparison of the computed and predicted Nusselt number values of these three cases indicates that the effects of convex-surface curvature and supercritical state of the working fluid on the convection heat transfer rate are of the same order of magnitude for the geometry/flow configurations considered.

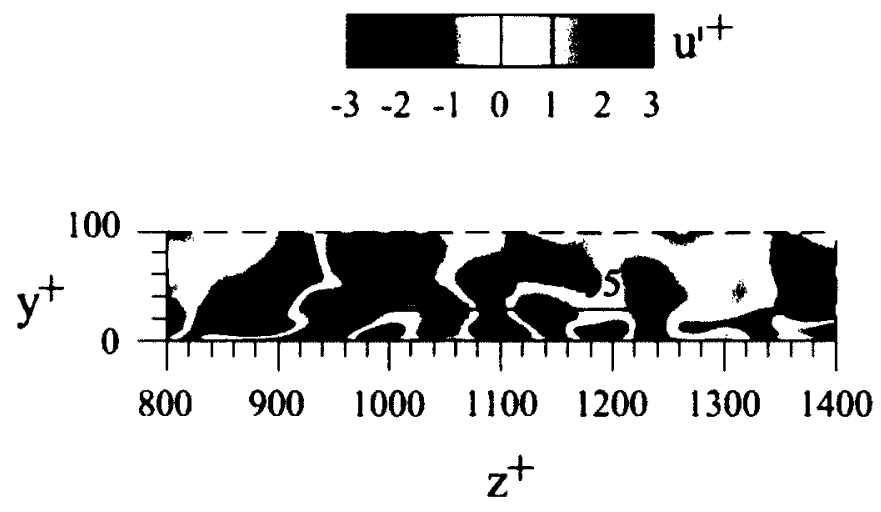

(a)

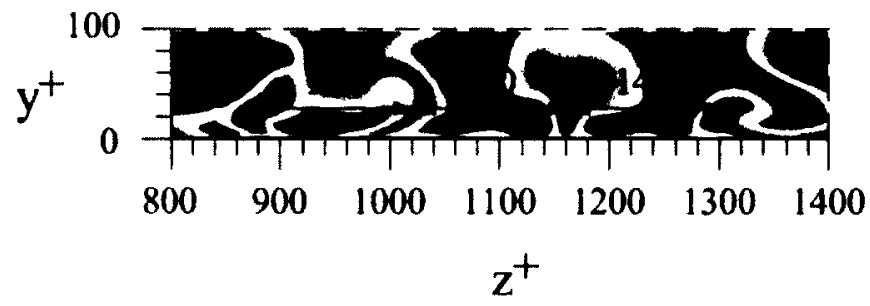

(b)

Figure 4.25: Spanwise streak spacing observed in the distribution of streamwise component of fluctuation velocity for (a) Case 3; (b) Case 4. 
The rate of turbulent mixing may be analyzed through the production $\left(P^{+}\right)$and dissipation $\left(\varepsilon^{+}\right)$rates of turbulence kinetic energy, which are plotted in Figure 4.26 for all test cases listed in Table 4.1. Analysis of the unheated test cases reveals that the mean rate of production of turbulence kinetic energy is reduced for Case 2 when compared to Case 1 , especially in the region with the highest turbulence production rates. This is expected since the radial-equilibrium mechanism due to the streamwise curvature works against wall-normal motions, and it is well established that such motions, which are related to the presence of hairpin-like vortical structures and associated near-wall streaks, significantly contribute to turbulence production (Zhou et al., 1999; Adrian et al., 2000).

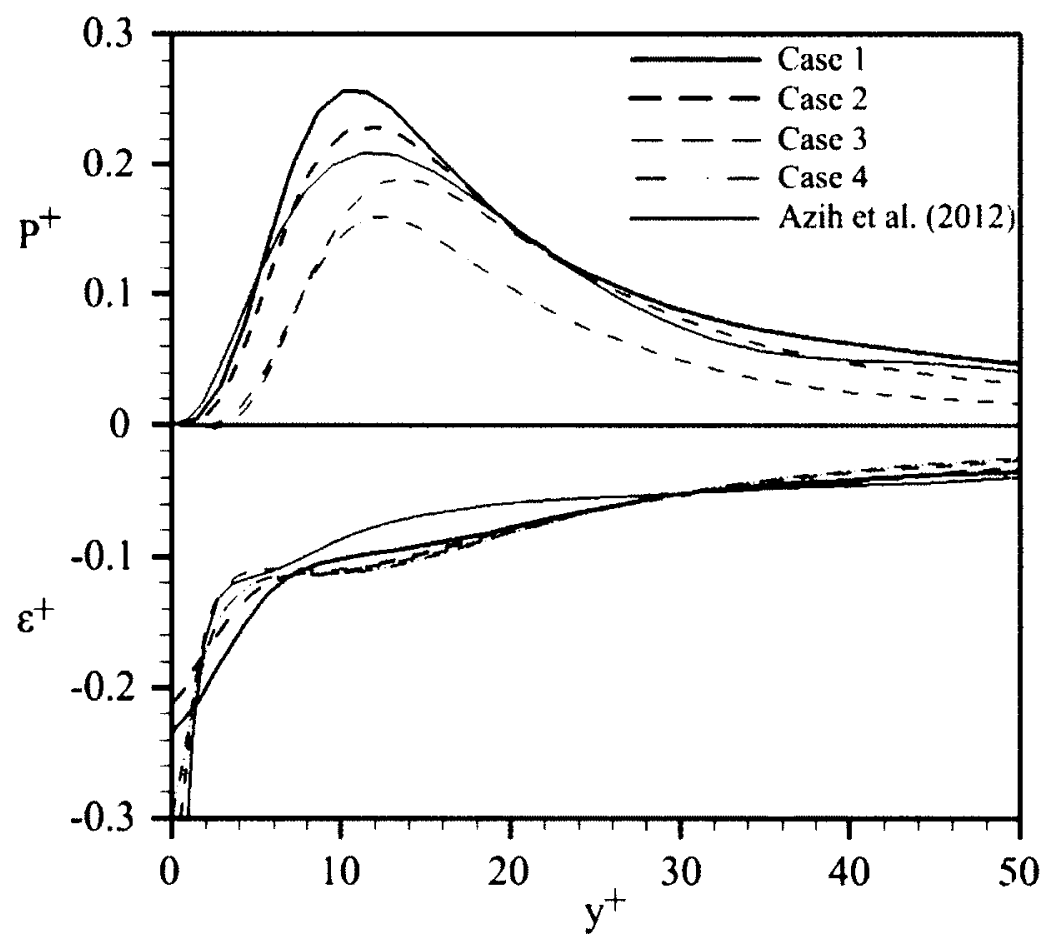

Figure 4.26: Mean rates of turbulence kinetic energy production and dissipation for Cases 1 and 2 (black lines), Case 3, Case 4 and data of Azih et al. (2012) (red lines).

To capture the additional effects of wall-normal property variations associated with the supercritical thermodynamic state, Figure 4.26 compares the results of Cases 3 
and 4 to those of Azih et al. (2012) for a flat surface. It should be noted here that Azih et al. (2012) simulated flow over a flat-surface with $q / G$ values of 0.9 and 1.2 , and at a Reynolds number of $R e_{\tau}=250$. For their case with $q / G=0.9$, the wall-normal thermophysical property gradients that occur when a supercritical-fluid flow is heated was demonstrated to cause improved-heat-transfer as compared to their case with $q / G=1.2$ in which normal-heat-transfer was observed. In particular, at the conditions in which they observed improved-heat-transfer, the viscosity decreased at a lower rate as the wall was approached, thereby promoting inviscid instabilities and thus resulting in more turbulence kinetic energy production between the wall and the wall-normal location of the peak $P^{+}$value.

When the turbulence kinetic energy production rates for Case 3, Case 4, and the study of Azih et al. (2012) corresponding to $q / G=0.9$ are compared, a decrease in the peak turbulence kinetic energy production rate is noted with increasing convex surface curvature which is consistent with the results from the unheated cases of the present study. In addition, the case of Azih et al. (2012) shows an increase in the production rate in the region between the wall and wall-normal location corresponding to the peak value of $P^{+}$when compared to both Cases 3 and 4 of the present study that show similar $P^{+}$ values in that region. This observation suggests that the presence of streamwise surface curvature suppresses the mechanisms responsible for improved heat transfer, and therefore confirms the earlier inference based on the predicted and computed Nusselt numbers, that the effect of streamwise convex surface curvature and the supercritical state of the working fluid on forced-convection heat transfer rate are of the same order of magnitude for the flow/geometry configurations considered in this study. 


\section{Chapter: Conclusions}

The use of water at the supercritical thermodynamic state as a coolant in the supercritical water-cooled reactor is proposed with wire-wrap spacers on the fuel rods which are known to enhance the heat transfer from the fuel rods to the supercritical coolant by enhancing the turbulence activity in the flow. The wire-wrap spacers impose streamwise convex surface curvature on the flow as it develops over the surface of the fuel rods and therefore in the present study, direct numerical simulations have been used to investigate the effect of streamwise convex surface curvature on the coherent flow structures in the turbulent boundary layer. The streamwise convex surface curvature is observed to have similar effects on turbulence with spatially uniform fluid properties and with strong wall-normal property variations associated with the supercritical thermodynamic state. In the case of spatially uniform fluid properties, the stabilizing effects of streamwise convex surface curvature, which exist due to the radial equilibrium between wall-normal pressure gradients and centrifugal forces in the boundary layer, cause a decrease in the streamwise spacing of individual hairpin-like vortical structures of turbulence within a wave packet. The promotion of stability by the radial equilibrium mechanism also causes an increase in the spanwise spacing of hairpin-like coherent vortical structures. These vortical structures drive the wall-normal momentum and energy exchange in the turbulent boundary layer. Correspondingly, the rate of turbulence kinetic energy production is reduced with an increase in the degree of streamwise convex curvature and thereby results in lower heat transfer rates in the case of strong wall-normal property variations of water at the supercritical thermodynamic state. For the present flow 
and geometric configurations considered, the reduction in heat transfer rate due to the stabilizing effects of streamwise convex surface curvature is observed to be of the same order of magnitude as the improvement in heat transfer rate brought about by the supercritical thermodynamic state of the working fluid. 


\section{Chapter: Recommendations for Future Work}

The use of wire-wrap spacers on the fuel rods in nuclear fuel bundles is known to enhance the heat transfer rates from the fuel rods to the coolant flow by enhancing the turbulence activity in the flow. These wire-wrap spacers impose streamwise convex surface curvature on the turbulent coolant flow and accordingly the research in this thesis established the effect of streamwise convex surface curvature on turbulence in terms of the coherent flow structures of turbulence. The stabilizing influence of streamwise convex surface curvature was seen to modify the behaviour of the coherent flow structures of turbulence and reduce the heat transfer rates in the boundary layer. In a nuclear fuel bundle scenario, although the presence of wire-wrap spacers force the turbulent coolant flow to experience streamwise convex surface curvature, they also induce a net transport of the coolant flow from one sub-channel into adjacent subchannels. In comparison to a fuel bundle without wire-wrapped fuel rods, the presence of wire-wrap spacers thus enhances the flow mixing and thereby the thermal mixing processes. Therefore, a proposed research would be to study the behaviour of coherent flow structures in a fuel bundle with wire-wrapped fuel rods using direct numerical simulations in conjunction with such a study of a fuel bundle without wire-wrap spacers to further understand the flow mixing process associated with wire-wrap spacers.

In a nuclear fuel bundle, the turbulent coolant flow is under the influence of an axial pressure gradient and due to the secondary motions induced by the wire-wrap spacers, transverse pressure gradients also exist in the fuel bundle. Thus, it would serve useful to study the effects of streamwise and spanwise pressure gradients in the presence 
of streamwise convex surface curvature on the behaviour of the coherent flow structures of turbulence. Such a study would aid in the interpretation of flow behaviour, for example, in a direct numerical simulation study of a fuel-rod bundle with wire-wrap spacers. 


\section{Appendices}

Appendix A : Review of Boundary-Layer Transition, Statistical Treatment of a Turbulent Boundary Layer and Effect of Convex Surface Curvature on Transition.

\section{A.1 Boundary Layers}

The existence of a boundary layer was first conceptualized by Prandtl (1904) when he proposed that the flow over a solid surface contains a very thin region next to the wall where viscous effects are significant in comparison to the rest of the flow, which can be modelled as inviscid. In a short period of time, boundary layer theory became the foundation of modern fluid dynamics due to its vast range of applicability. Most boundary layer flows of practical importance are turbulent in nature and therefore a significant amount of research has been devoted to the understanding and modelling of such flows (Schlichting, 1979). Flow visualization experiments and direct numerical simulations (DNS) of turbulent flows have shown the existence of coherent structures in the boundary layer which play a significant role in the generation and preservation of turbulence (Carlier and Stanislas, 2005). This section is a short description of laminar-toturbulent transition.

\section{A.1.1 Laminar-to-Turbulent Transition in Boundary Layer Flows}

The physical mechanisms contributing to the processes that promote laminar-toturbulent transition are complex and depend on several factors such as freestream turbulence, pressure-gradient, flow-oscillation, wall roughness, suction and blowing, and wall curvature; such factors impose disturbances on a laminar flow (Schlichting, 1979). According to Morkovin (1969), transition from laminar to turbulent flow can be grouped 
into two classes. In the first class of transition, the breakdown to turbulent flow can be said to comprise of three main stages: receptivity, linear stability and non-linear breakdown as shown in Figure A.1 (Kachanov, 1994). The process by which external disturbances enter the boundary layer as instability waves was referred by Morkovin (1969) as receptivity. At the receptivity stage, in the region of relatively low local Reynolds number, the external disturbances transformed into boundary layer perturbations are referred to as the Tollmien-Schlichting (T-S) waves (Schubauer and Skramstad, 1947).

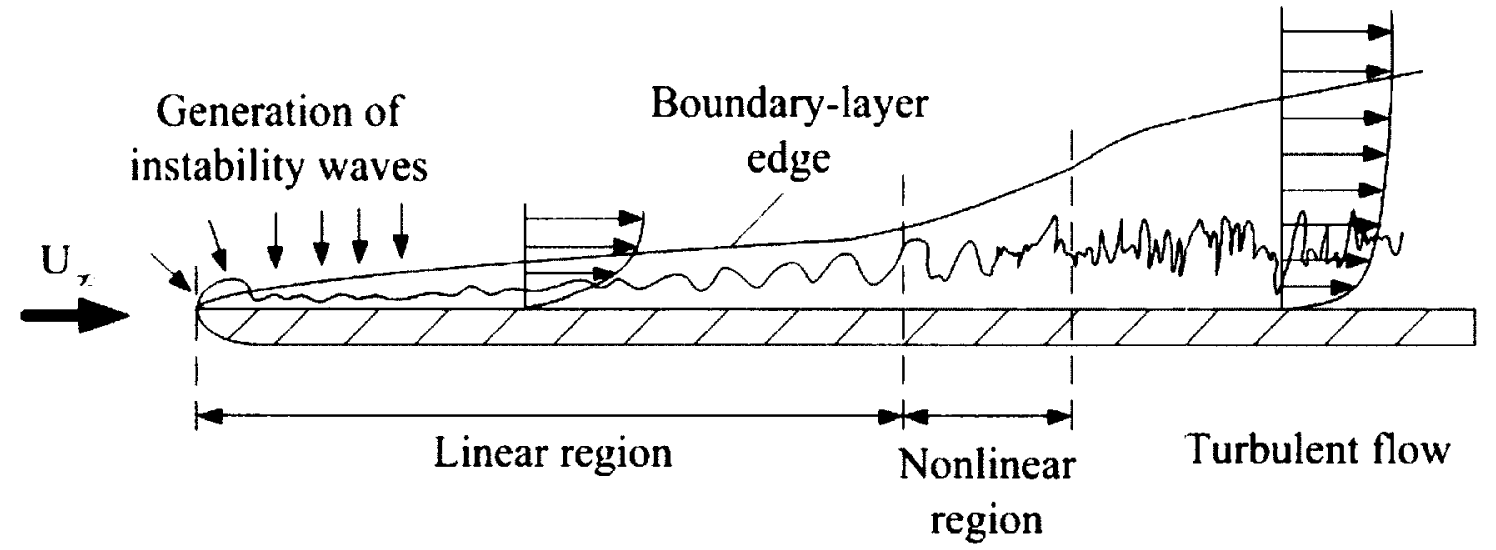

Figure A.1: Process of transition from laminar to turbulent flow (adapted from Kachanov, 1994).

If the initial amplitude of the T-S waves is small relative to the freestream velocity, then the development of these waves can be described by the linear stability theory. The region of the flow where the linear stability theory applies is called as the linear region which is usually quite extensive as compared to the non-linear region in the first class of transition as observed in Figure A.1. Instabilities that can be described by the linear stability theory are referred to as primary instabilities and are governed by the Orr-Sommerfeld equation (Herbert, 1988) which is derived by linearizing the two 
dimensional (2-D) form of the governing fluid-flow equations and applying a sinusoidal disturbance to it (Orr, 1907 and Sommerfeld, 1909). The Orr-Sommerfeld equation is a fourth-order, partial differential equation whose solution, developed by Tollmien (1929) and Schlichting (1933), was verified experimentally by Liepmann (1943) and Schubauer and Skramstad (1947). Solution of the Orr-Sommerfeld equation reveals a critical Reynolds number that represents a threshold value above which at least some of the T-S waves in the boundary layer are amplified and lead to transition. When the T-S waves are amplified on the order of $1-2 \%$ of the freestream velocity, non-linear interactions occur in the form of secondary instabilities which are characteristic of the turbulence phenomenon (Söylemez and Özkol, 2003).

In the second class of transition known as bypass transition, the relatively higher amplitude of the T-S waves results in immediate non-linear interactions, and cause the laminar-flow breakdown to turbulent-flow. Thus, the linear stability theory is not applicable for this case. The pathways of transition to turbulence can be represented by Figure A.2. During flow transition, experimental and numerical studies have shown the occurrence of organized motions in the boundary layer irrespective of the pathway taken to transition to turbulent flow (Kachanov, 1994). Such organized motions strongly contribute to the development of the transitioning and turbulent boundary layers and therefore require a detailed study; but prior to studying the structure and interaction of these organized motions, it would be beneficial to review the statistical treatment of a turbulent boundary layer conducted historically. 


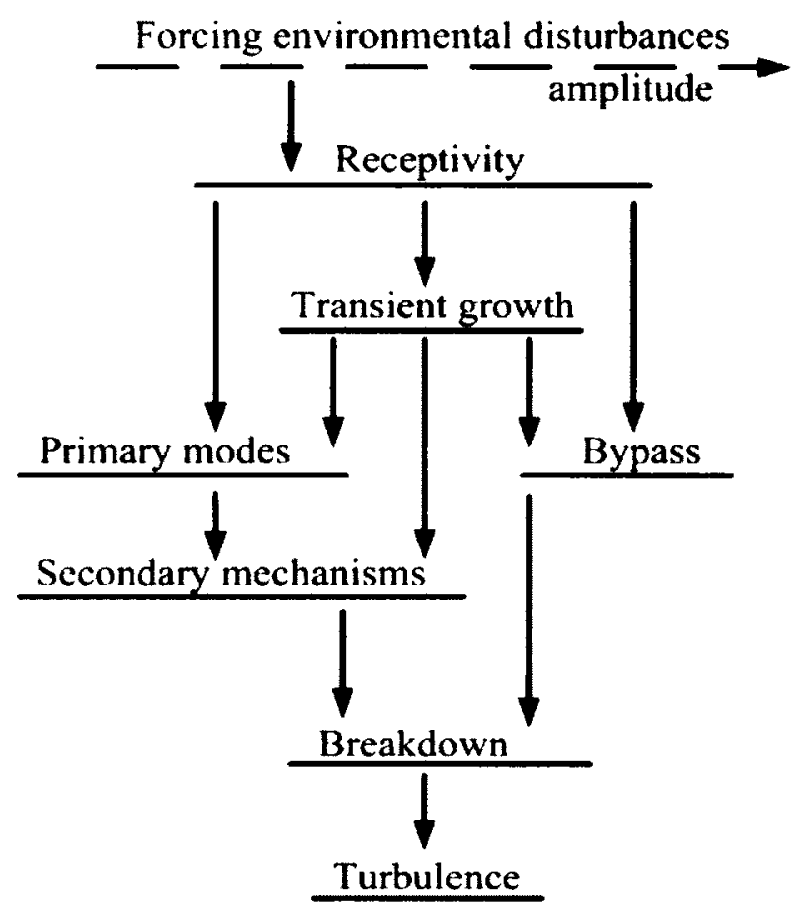

Figure A.2: Roadmap to turbulence (adapted from Saric et al., 2002).

\section{A.1.2 Wall-Bounded Turbulent Boundary Layer}

The phenomenon of turbulence is extremely complex due to the wide range of spatial and temporal scales present in the flow. Several practical applications involve turbulent fluid flow (such as the coolant flow through a nuclear fuel bundle, flow past aircraft wing surfaces, etc.), hence the need to precisely predict the behaviour of such flows. The first significant attempt in this direction was the work of Osborne Reynolds in 1895 who decomposed the velocity field of a turbulent flow into a mean and fluctuating part as follows:

$$
u_{i}=\bar{u}_{i}+u_{i}^{\prime}
$$

where $u_{i}$ is the instantaneous velocity vector, $\bar{u}_{i}$ is the time-averaged velocity vector, $u_{i}^{\prime}$ is the instantaneous fluctuating velocity vector and the subscript $i=1,2,3$ represents the streamwise $(x)$, wall-normal $(y)$, and spanwise $(z)$ coordinate directions, respectively. A 
similar decomposition is also applied to the scalar quantities such as pressure and temperature. These decompositions can be substituted into the Navier-Stokes (N-S) equation to obtain the N-S equation in terms of the average and fluctuating quantities. The N-S equations, which are the governing equations for fluid flow, are treated in more detail in Chapter 3 of this thesis. After substitution, the N-S equations are averaged in time resulting in the Reynolds averaged Navier-Stokes (RANS) equations that are similar in form to the original N-S equation with the exception of an additional term that is obtained due to averaging of the product of velocity fluctuations. This additional term is known as the convective stress term (Cantwell, 1981) or more commonly known as the Reynolds stress term, which is shown in the following equation in the tensor notation:

$$
R_{i j}=\overline{\rho u_{j}^{\prime} u_{\imath}^{\prime}}
$$

where $R_{i j}$ is the Reynolds stress tensor and $\rho$ is the density, and the overbar signifies time-averaged quantities. Several semi-empirical theories have been developed that attempt to determine the Reynolds stress term which is referred to as the "closure" problem in turbulence literature. The mixing-length theory developed by Prandtl (1925) is the first such theory that relates the Reynolds stresses to the mean flow field by an effective eddy viscosity or mixing length to close the system of equations. More sophisticated models have since been developed such as the one equation SpalartAllmaras model or the two equation turbulence kinetic energy-turbulence dissipation (kع) model among others to determine the effective eddy viscosity. According to Cantwell (1981), such mathematical models for turbulence were based on the concept of an "eddy" but there were hardly any attempts to visualize this turbulent motion. The development of 
a suitable theory to predict turbulent flows requires a substantial understanding of the physics of such flows in terms of the structure of the turbulent boundary layer.

Before attempting a study of the structure in a turbulent boundary layer, it would be beneficial to review the behaviour of the mean velocity profile which is also an important indicator of the structure of wall-bounded turbulent boundary layers. Traditionally, the turbulent boundary layer for an unheated flow has been divided into regions as shown in Table A.1 where the superscript "+" denotes non-dimensional quantities normalized by the wall variables, viscosity $(v)$ and friction velocity $\left(u_{\tau}\right)$, defined as follows:

$$
\begin{gathered}
v=\frac{\mu}{\rho} \\
u_{\tau}=\sqrt{\frac{\tau_{w}}{\rho}} \\
\left.\tau_{w}=\mu \frac{\partial \bar{u}}{\partial y}\right]_{y=0} \\
\delta_{v}=\frac{v}{u_{\tau}} \\
y^{+}=y \frac{u_{\tau}}{v} \\
u^{+}=\frac{\bar{u}}{u_{\tau}}
\end{gathered}
$$


where,

$\mu \quad: \quad$ Dynamic viscosity $(\mathrm{kg} / \mathrm{m} \mathrm{s})$

$\rho:$ Density $\left(\mathrm{kg} / \mathrm{m}^{3}\right)$

$v \quad: \quad$ Kinematic viscosity $\left(\mathrm{m}^{2} / \mathrm{s}\right)$

$\tau_{w}:$ Shear stress at the wall $(\mathrm{Pa})$

$u_{\tau}:$ Friction velocity $(\mathrm{m} / \mathrm{s})$

$\delta_{v}:$ Viscous length scale $(\mathrm{m})$

$y$ : Wall-normal distance $(\mathrm{m})(y=0$ represents at the wall)

$\bar{u}:$ Mean streamwise velocity $(\mathrm{m} / \mathrm{s})$

Table A.1: Boundary layer division based on mean velocity profile (Pope, 2000)

\begin{tabular}{|c|c|c|c|}
\hline Region & Location & Property of Region & Defining Equation \\
\hline Inner layer & $\frac{y}{\delta}<0.1$ & $\begin{array}{c}\text { Mean velocity } \\
\text { dependent on the } \\
\text { inner variables, } u_{\tau} \\
\text { and } \delta_{\gamma}\end{array}$ & \\
\hline Viscous wall region & $y^{+}<50$ & $\begin{array}{c}\text { Significant viscous } \\
\text { effects }\end{array}$ & \\
\hline Viscous sub-layer & $y^{+}<5$ & $\begin{array}{c}\text { Viscous stress } \\
\text { dominates over } \\
\text { Reynolds shear } \\
\text { stress } \\
\end{array}$ & $u^{+}=y^{+}$ \\
\hline Buffer layer & $5<y^{+}<30$ & $\begin{array}{c}\text { Region between the } \\
\text { viscous sub-layer } \\
\text { and the log-law } \\
\text { region }\end{array}$ & \\
\hline $\begin{array}{l}\text { Log-law region } \\
\text { (Law of the wall) }\end{array}$ & $y^{+}>30, \frac{y}{\delta}<0.3$ & $\begin{array}{l}\text { Region where the } \\
\text { law of the wall is } \\
\text { applicable }\end{array}$ & $u^{+}=\frac{1}{\kappa} \ln y^{+}+B$ \\
\hline Overlap region & $y^{+}>50, \frac{y}{\delta}<0.1$ & $\begin{array}{l}\text { Region overlaps } \\
\text { between the inner } \\
\text { and outer layers }\end{array}$ & \\
\hline Outer layer & $y^{+}>50$ & $\begin{array}{c}\text { Viscosity effects are } \\
\text { negligible. }\end{array}$ & \\
\hline
\end{tabular}


The viscous effects are important in the inner layer, while the outer layer is dominated by inertial effects. The inner layer is normally scaled by $v$ and $u_{\tau}$ and the outer region is normally scaled by the boundary-layer thickness $(\delta)$ and the freestream velocity of the flow. In the log-law region, the logarithmic dependence of the mean velocity on the wallnormal coordinate is observed in a wide range of wall-bounded turbulent flows. In the defining equation for the log-law region, $\kappa$ and $B$ are constants that are usually within $5 \%$ of $\kappa=0.41$ and $B=5.2$, where $\kappa$ is also known as the von Kármán constant. Smith et al. (1991) mention that the classification of the boundary layer into regions such as those shown in Table A.1 are based on the mean velocity profile and not the dynamical features of turbulence, which are believed to sustain turbulence. In a review of turbulent boundary layers by Cantwell (1981), the author observes that the maximum production of turbulence kinetic energy occurs in the buffer layer and the inner layer contributes to nearly half of the total production of turbulence kinetic energy. Such features of the turbulent boundary layer could not be explained with traditional averaging methods which compelled many researchers to examine the structure of the turbulent boundary layer in order to understand the dynamics of the flow. 


\section{A.2 Effect of Streamwise Convex Surface Curvature on Shear-Layer Transition}

The stabilizing influence of streamwise convex surface curvature dampens the disturbances in the boundary layer (Ramaprian and Shivaprasad, 1978) causing the critical Reynolds number to shift higher than the critical Reynolds number applicable for the laminar boundary layer on a flat surface. This was experimentally observed by Liepmann (1943) in a detailed experiment with two different degrees of surface curvatures, $\delta / R=0.00075$ and $\delta / R=0.005$. The critical Reynolds number increases as the degree of surface curvature in increased (synonymous with a decrease in the radius of curvature) for the convex surface but Liepmann (1943) found that convex-surface curvature in the range used in his experiments did not have an appreciable effect on transition as compared to the flat surface. The stabilizing influence of convex-surface curvature can also be expected to delay the transition to turbulence which would result in a higher shape factor and a lower intermittency factor compared to a flat surface (Ozalp and Umur, 2003; Umur and Ozalp, 2006). These authors also observe a lower magnitude of the Stanton number on the convex-surface as compared to the flat-surface case. The Stanton number $(S t)$ is given as:

$$
S t=\frac{h}{\left(\rho u_{\infty} C_{p}\right)}
$$

where $h$ is the convection heat transfer coefficient, $u_{\infty}$ is the freestream velocity and $C_{p}$ is the specific heat at constant pressure. This implies that in comparison to a flat surface, convex surface curvature reduces the heat transfer across a transitioning boundary layer. 


\section{Appendix B : Effect of Mildly-Curved and Strongly-Curved Streamwise Convex Surface on an Initial Disturbance.}

\section{B.1 Evolution of an Initial Disturbance on a Strongly-Curved Streamwise Convex Surface}

This section illustrates the effect of strongly-curved streamwise convex surface on the initial disturbance used in the flow and compares the evolution of a similar disturbance on a flat surface. The $\delta / R$ value of the strongly-curved convex surface is 25 times that of the mildly-curved convex surface Case 2 at the same boundary layer thickness. In the following discussion, the streamwise and spanwise coordinates are normalized by the test-surface length, $L$, and the wall-normal coordinate is normalized by the boundary layer thickness of the flow. The boundary layer thickness for the flat surface case is calculated based on the location where the mean streamwise velocity is $99 \%$ of the freestream velocity. The boundary layer edge velocity for the convex surface case is evaluated at the point where the slope of the mean streamwise velocity profile matches the slope of the initial radial distribution of velocity since this case is initialized with a radially linear distribution of streamwise velocity. Time is normalized by $\delta / u_{\alpha}$ $\left(t^{n}=t u_{x} / \delta\right)$, where $u_{\infty}$ is the boundary layer edge velocity and $\delta$ is the boundary layer thickness of the flow. The quantities are normalized as mentioned because the flow over the convex surface does not attain a fully turbulent state but is relaminarized due to the stabilizing effects of strong convex curvature.

The disturbance is created with two point sources placed near the wall that introduce mass into the boundary layer at a velocity that is $250 \%$ of the freestream velocity at a momentum-thickness Reynolds number of $R e_{\theta}=650$. This disturbance for 
both the flat and convex surfaces is shown in Figure B.1 and revealed using an isosurface of the second invariant of the velocity gradient tensor $(Q)$ coloured according to the wall-normal height. It should be mentioned that the magnitude of the velocity with which mass was introduced into the flow using the point sources was varied from $25 \%$ to $250 \%$ of the freestream velocity and the results presented are for the strongest magnitude of the disturbance. Figure B.1 shows that the initial disturbance used was the same for both the flat surface and the convex surface cases.

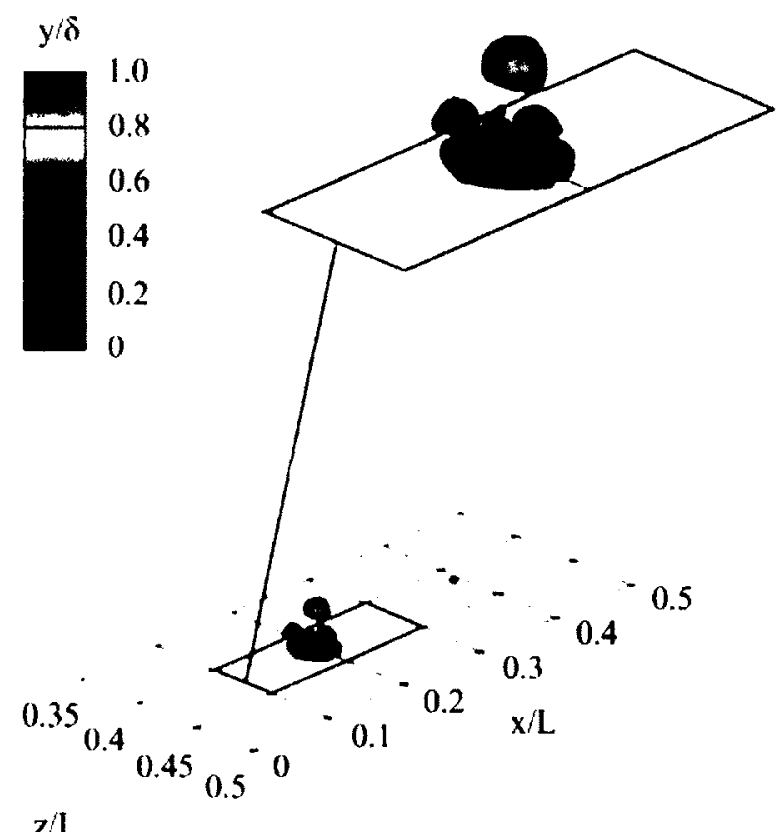

(a)

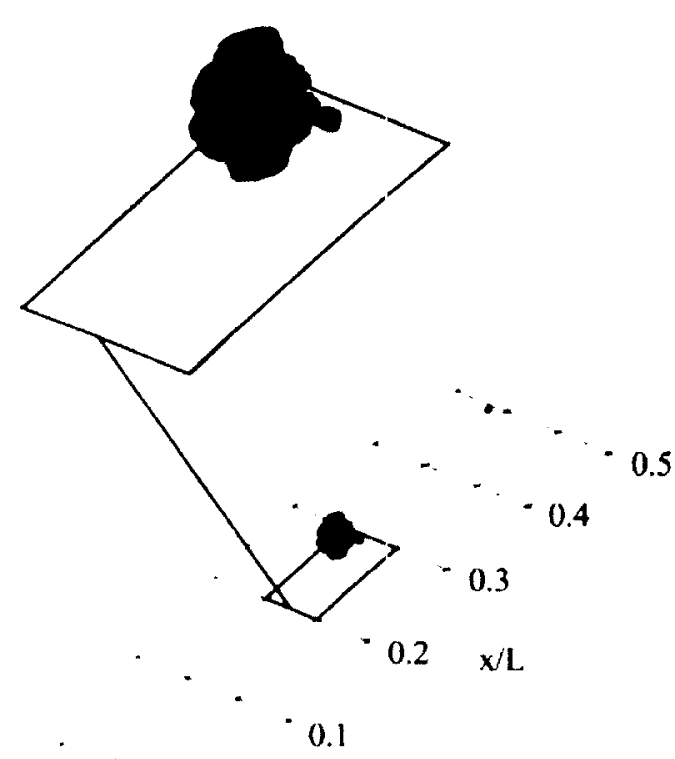

${ }^{0.35} 0.4_{0.45} 0.5$

$\mathrm{z} / \mathrm{L}$

(b)

Figure B.1: Initial disturbance on the (a) flat surface and (b) convex surface.

Further evolution of the disturbance is shown in Figure B.2, Figure B.3 and Figure B.4. A detailed analysis of the development of the structures was not carried out as the primary objective of this thesis is the study of the effect of convex surface curvature on turbulent boundary layers. The relaminarization of the flow even with strong 
perturbations suggests that the critical Reynolds number of the flow was not reached for this case and also suggests that the critical Reynolds number increases with an increase in the curvature (or $\delta / R$ ratio) of the surface. Some features of the development of the disturbance common to the mildly-curved convex surface Case 2 include the roll-up of an unstable shear layer due to the creation of an inflectional point in the velocity profile by the disturbance sources. This leads to the formation of the largest hairpin-like structures observed in both the cases. The interaction with the mean shear stretches the largest hairpin-like structures for both cases but the structures on the flat surface stretch more and rise further towards the freestream than those on the convex surface. The is due to the strong vorticity of the head and legs of the structures which increases with increased stretching by the mean shear elongating the legs and causing the structures to induce them into the higher momentum regions on the flat surface case. On the strongly-curved convex surface, interactions with the mean shear occur under the stabilizing influence of the radial equilibrium mechanism. Figure B.4 indicates that the structures on the convex surface case are already dissipating under this stabilizing influence and by $t^{n}=25$, the flow is observed to be completely laminarized. 


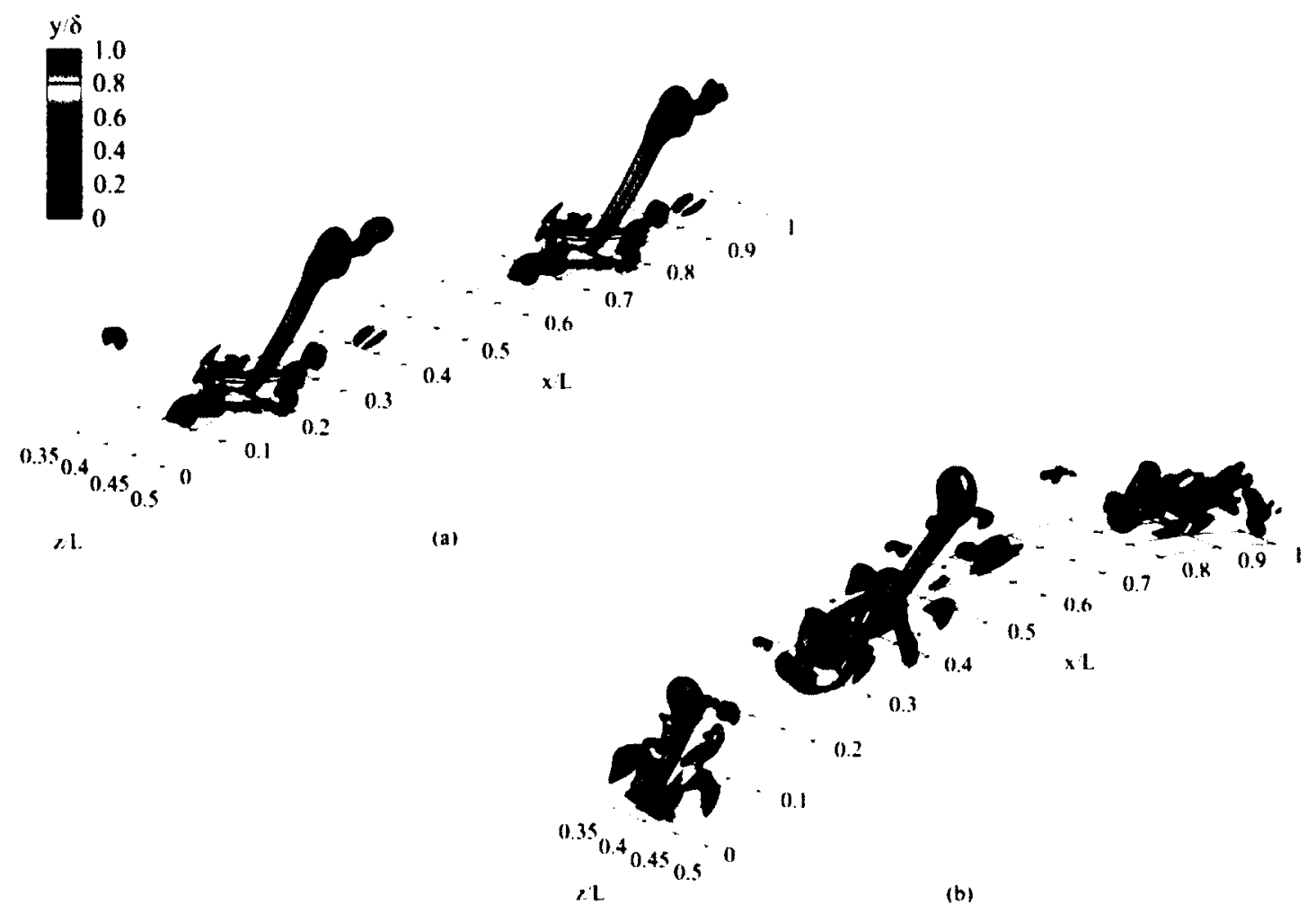

Figure B.2: Evolution of the initial disturbance compared at $t^{n}=1.5$ : (a) flat surface and (b) convex surface. 


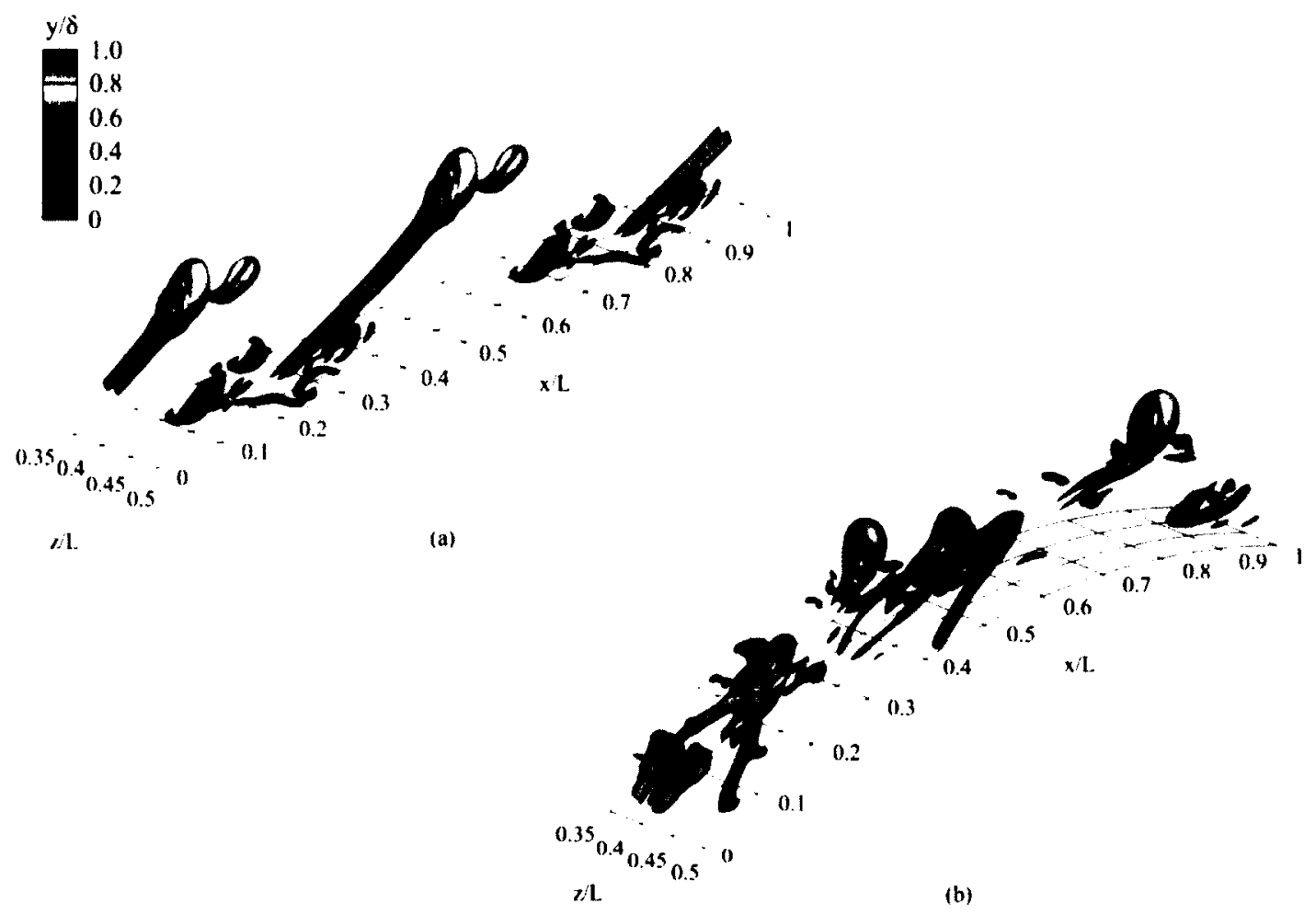

Figure B.3: Evolution of the initial disturbance compared at $t^{n}=3$ : (a) flat surface and (b) convex surface. 


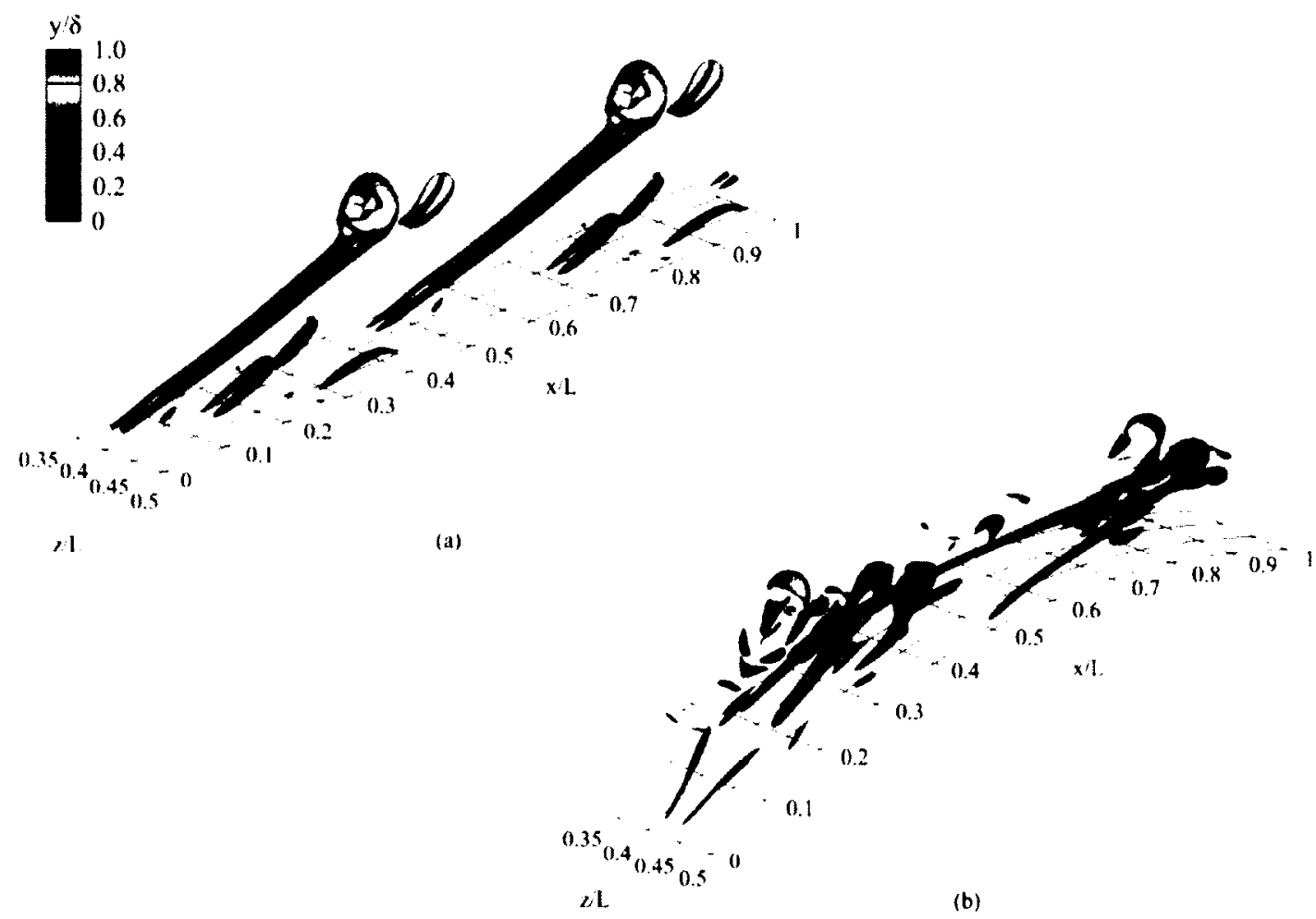

Figure B.4: Evolution of the initial disturbance compared at $t^{n}=5$ : (a) flat surface and (b) convex surface. 


\section{B.2 Comparison of Coherent Structures in the Turbulent Boundary Layer on a Mildly-Curved Convex Surface Disturbed using Two Disturbance Duration Ranges}

This section compares the properties of the coherent structures within the fully turbulent boundary layer on the mildly-curved convex surface Case 2 developed using two different mass-injection disturbance duration ranges. In both cases, the velocity at which the mass is injected has a magnitude that is $85 \%$ of the freestream velocity but the duration for which the disturbance is activated for one case is $\Delta t^{+}=100$ and for the other case is $\Delta t^{+}=17.5$. The normalized time $\Delta t^{+}=\Delta t u_{\tau}^{2} / v$, where $u_{\tau}$ is the friction velocity and $v$ is the kinematic viscosity. The case for which the duration is $\Delta t^{+}=100$ will be referred to as Case 2-1 and the case for which the duration is $\Delta t^{+}=17.5$ will be referred to as Case $2-2$ in this section for simplicity.

The comparison between the two cases is presented in terms of the streamwise spacing of hairpin vortices in a wave packet and the spanwise spacing of the hairpin vortices; the two properties which exhibited significant differences from the flat surface Case 1. Figure B.5 shows that the streamwise spacing between individual hairpin vortices in a wave packet is between 100-150 wall units for both Case 2-1 and Case 2-2. Figure B.6 shows that the average streak spacing visualized through the instantaneous fluctuation distribution of the streamwise velocity component is the same for both the cases (around 110 wall units). This shows that while the boundary layers for Cases 2-1 and 2-2 were disturbed differently, it did not affect the state of the fully turbulent boundary layer. 


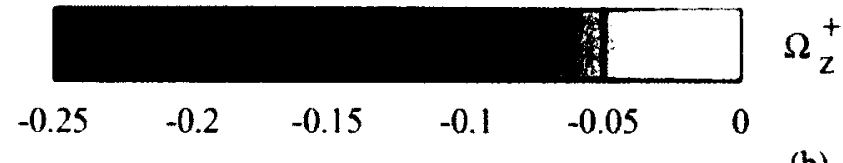

(a)
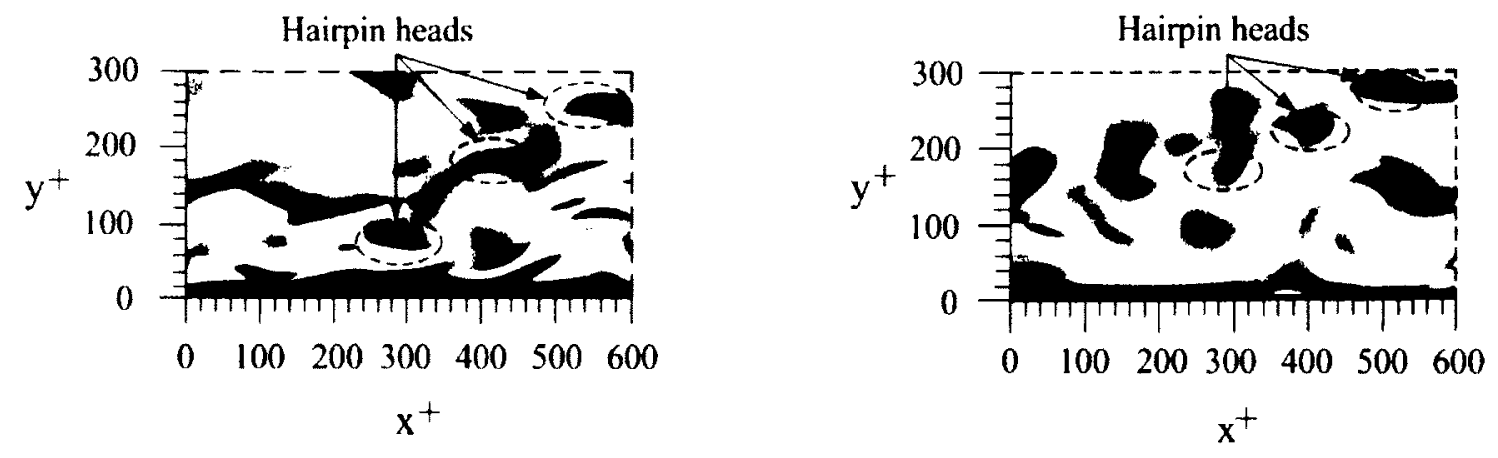

Figure B.5: Streamwise spacing of hairpin vortices in a wave packet for (a) Case 21; (b) Case 2-2 visualized through flood plots of spanwise component of vorticity.
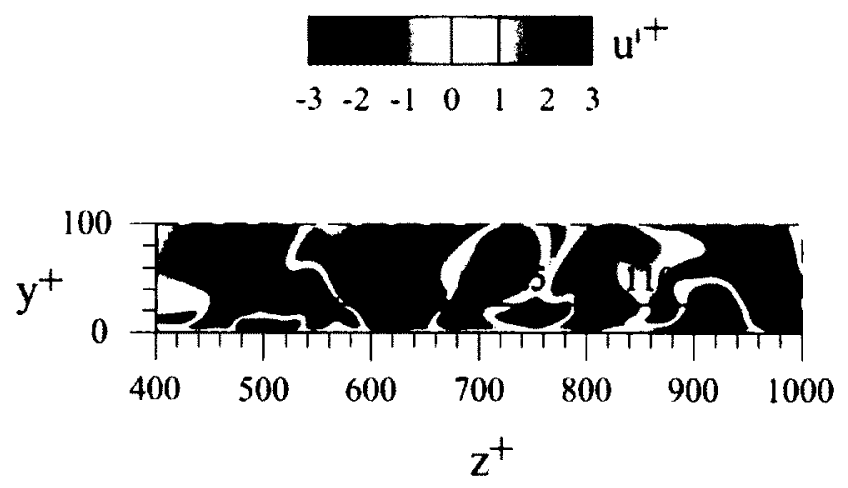

(a)

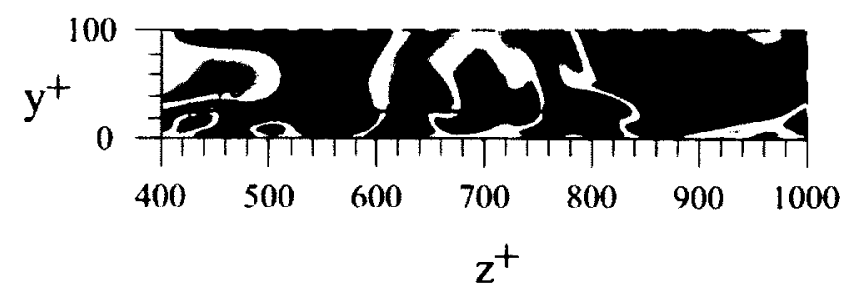

(b)

Figure B.6: Spanwise streak spacing observed in the distribution of streamwise component of fluctuation velocity for (a) Case 2-1; (b) Case 2-2. 


\section{References}

Acarlar, M. S. and Smith, C. R. (1987), "A study of hairpin vortices in a laminar boundary layer. Part 2. Hairpin vortices generated by fluid injection," Journal of Fluid Mechanics, 175, pp. 43-83.

Adrian, R. J., Meinhart, C. D. and Tomkins, C. D. (2000), "Vortex Organization in the outer region of the turbulent boundary layer," Journal of Fluid Mechanics, 422, pp. $1-54$.

Adrian, R. J. (2007), "Hairpin vortex organization in wall turbulence," Physics of Fluids, 19, 041301-16.

Ahmad, I. and Kim, K. (2006), "Flow and Convective Heat Transfer Analysis Using RANS for a Wire-Wrapped Fuel Assembly," Journal of Mechanical Science and Technology, 20, No. 9, pp. 1514-1524.

Alving, A. E., Smits, A. J. and Watmuff, J. H. (1990), "Turbulent boundary layer relaxation from convex curvature," Journal of Fluid Mechanics, 211, pp. 529-556.

ANSYS (2009), ANSYS CFX-Solver Theory Guide, 12 ed., ANSYS, inc, 275 Technology Drive, Canonsburg, PA.

Azih, C., Brinkerhoff, J. R. and Yaras, M. I. (2012), "Direct numerical simulation of convective heat transfer in a zero-pressure-gradient boundary layer with supercritical water," Journal of Thermal Science, 21, pp. 49-59. 
Bae, J. H., Yoo, J. Y. and Choi, H. (2005), "Direct numerical simulation of turbulent supercritical flows with heat transfer," Physics of Fluids, 17, 105104-1-24.

Bernard, P. S., Thomas, J. M. and Handler, R. A. (1993), "Vortex dynamics and the production of Reynolds stress," Journal of Fluid Mechanics, 253, pp. 385-419.

Bishop, A. A. and Todreas, N. (1980), "Hydraulic characteristics of wire-wrapped rod bundles," Nuclear Engineering and Design, 62, pp. 271-293.

Bradshaw, P. (1969), "The analogy between streamline curvature and buoyancy in turbulent shear flow," Journal of Fluid Mechanics, 36, part 1, pp. 177-191.

Brinkerhoff, J. R. and Yaras, M. I. (2011), "Direct numerical simulation of a square jet ejected transversely into an accelerating, laminar main flow," In: Proceedings of the $7^{\text {th }}$ International Symposium on Turbulence and Shear Flow Phenomena, Ottawa, Canada July $28-31$.

Brinkerhoff, J. R. and Yaras, M. I. (2011), "Direct numerical simulation of the growth of coherent flow structures in a triggered turbulent spot," In: Proceedings of the $7^{\text {th }}$ International Symposium on Turbulence and Shear Flow Phenomena, Ottawa, Canada July $28-31$.

Brooke, J. W. and Hanratty, T. J. (1993), "Origin of turbulence producing eddies in a channel flow," Physics of Fluids, 5, No. 4, pp. 1011-1022. 
Buschmann, M. H., Indinger, T. and Gad-el-Hak, M. (2009), "Near-wall behaviour of turbulent wall-bounded flows," International Journal of Heat and Fluid Flow, 30, pp. 996-1003.

Butler, K. M. and Farrell, B. F. (1993), "Optimal perturbations and streak spacing in wall-bounded turbulent shear flow," Physics of Fluids, 5, pp. 774-777.

Cantwell, B. J. (1981), "Organized Motion in Turbulent Flow," Annual Review of Fluid Mechanics, 13, pp. 457-515.

Carlier, J. and Stanislas, M. (2005), "Experimental study of eddy structures in a turbulent boundary layer using particle image velocimetry," Journal of Fluid Mechanics, $\mathbf{5 3 5}$, pp. 143-188.

Chernyshenko, S. I. and Baig, M. F. (2005), "The mechanism of streak formation in nearwall turbulence," Journal of Fluid Mechanics, 544, pp. 99-131.

Chiwanga, S. C. and Ramaprian, B. R. (1993), "The Effect of Convex Wall Curvature on the Large-Scale Structure of the Turbulent Boundary Layer," Experimental Thermal and Fluid Science, 6, pp. 168-176.

Chun, M. and Seo, K. (2001), "An experimental study and assessment of existing friction factor correlations for wire-wrapped fuel assemblies," Annals of Nuclear Energy, 28, pp. 1683-1695. 
Churchill, S. W. (1976), "A comprehensive correlating equation for forced convection from flat plates," Journal of American Institute of Chemical Engineers, 22, pp. 264268.

Fasel, H. F. (2006), "Instability and Transition in Boundary Layers: Direct Numerical Simulations," IUTAM Symposium on One Hundred Years of Boundary Layer Research, Meier, G. E. A. and Sreenivasan, K. R. (eds.), Springer, pp. 257-267.

Fischer, P., Lottes, J., Siegel, A. and Palmiotti, G. (2007), "Large Eddy Simulation of Wire-Wrapped Fuel Pins I: Hydrodynamics in a Periodic Array," Joint International Topical Meeting on Mathematics and Computation and Supercomputing in Nuclear Applications, California, USA.

Friedrich, R., Huttl, T. J., Manhart, M. and Wagner, C. (2001), "Direct numerical simulation of incompressible turbulent flows," Computers and Fluids, 30, pp. 555579.

Gajapathy, R., Velusamy, K., Selvaraj, P., Chellapandi, P. and Chetal, S. C. (2007), "CFD investigation of helical wire-wrapped 7-pin fuel bundle and the challenges in modelling full scale 217 pin bundle," Nuclear Engineering and Design, 237, pp. $2332-2342$.

Gibson, M. M., Verriopoulos, C. A. and Vlachos, N. S. (1984), "Turbulent boundary layer on a mildly curved convex surface Part 1: Mean flow and turbulence measurements," Experiments in Fluids, 2, pp. 17-24. 
Gibson, M. M. and Verriopoulos, C. A. (1984), "Turbulent boundary layer on a mildly curved convex surface Part 2: Temperature field measurements," Experiments in Fluids, 2, pp. 73-80.

Gillis, J. C. and Johnston, J. P. (1983), "Turbulent boundary-layer flow and structure on a convex wall and its redevelopment on a flat wall," Journal of Fluid Mechanics, 135, pp. 123-153.

Ginsberg, T. (1972), "Forced-Flow Interchannel Mixing Model for Fuel Rod Assemblies Utilizing a Helical Wire-Wrap Spacer System," Nuclear Engineering and Design, 22, pp. 28-42.

Gowda, B. H. L. and Narayana, P. A. A. (1980), "An experimental investigation of separating flow on a convex surface," Applied Scientific Research, 36, pp. 271288.

Gu, H. Y., Cheng, X. and Yang, Y. H. (2010), "CFD analysis of thermal-hydraulic behaviour of supercritical water in sub-channels," Nuclear Engineering and Design, 240, pp. 364-374.

Haidari, A. H. and Smith, C. R. (1994), "The generation and regeneration of single hairpin vortices," Journal of Fluid Mechanics, 277, pp. 135-162.

Head, M. R. and Bandyopadhyay, P. (1981), "New aspects of turbulent boundary-layer structure," Journal of Fluid Mechanics, 107, pp. 297-338. 
Herbert, T. (1988), "Secondary Instability of Boundary Layers," Annual Review of Fluid Mechanics, 20, pp. 487-526.

Hidalgo, P., Lang, A. W. and Thacker, W. D. (2006), "POD Study of the Coherent Structures within a Turbulent Spot," AIAA Paper no. 2006-1099.

Himmel, S., Class, A. G., Laurien, E. and Schulenberg, T. (2008), "CFD Analysis of Subchannel Flow in an HPLWR Assembly with Wire-Wraps," International Students Workshop on High Performance Light Water Reactors, Germany.

Hon, T. L. and Walker, J. D. A. (1987), "An analysis of the motion and effects of hairpin vortices," AFOSR Rep. FM-11, Dept. Mech. Eng. And Mech., Lehigh University; see also NASA Tech. Memo. 100858, ICOMP-88-9.

Honkan, A. and Andreopoulos, Y. (1997), "Vorticity, strain-rate and dissipation characteristics in the near-wall region of turbulent boundary layers," Journal of Fluid Mechanics, 350, pp. 29-96.

Hutchinson, B. R. and Raithby, G. D. (1986), "A Multigrid Method Based on the Additive Correction Strategy," Numerical Heat Transfer, 9, pp. 511-537.

IAPWS (2009), "Revised release on the IAPWS formulation 1995 for the thermodynamic properties of ordinary water substance for general scientific use," The International Association for the Properties of Water and Steam. 
IAPWS (2008), "Release on the IAPWS formulation 2008 for the viscosity of ordinary water substance," The International Association for the Properties of Water and Steam, Berlin, Germany, pp. 1-9.

Kachanov, Y. S. (1994), "Physical Mechanisms of Laminar-Boundary-Layer Transition," Annual Review of Fluid Mechanics, 26, pp. 411-482.

Karypis, G. and Kumar, V. (1995), "MeTiS Unstructured graph partitioning and sparse matrix ordering," Version 2.0, University of Minnesota, Minneapolis.

Kim, J., Moin, P. and Moser, R. (1987), "Turbulence statistics in fully developed channel flow at low Reynolds number," Journal of Fluid Mechanics, 177, pp. 133-166.

Kleiser, L. and Zang, T. A. (1991), "Numerical Simulation of Transition in WallBounded Shear Flows," Annual Review of Fluid Mechanics, 23, pp. 495-537.

Kline, S. J., Reynolds, W. C., Schraub, F. A. and Runstadler, P. W. (1967), "The structure of turbulent boundary layers," Journal of Fluid Mechanics, 30, pp. 741773.

Kline, S. J. and Robinson, S. K. (1989), "Quasi-coherent structures in the turbulent boundary layer. I. Status report on a community-wide survey of the data," Proceedings of the Zaric Memorial Conference on Near-Wall Turbulence, Kline, S. J. and Afgan, N. H. (eds.), pp. 200-217.

Liepmann, H. W. (1943), "Investigations on Laminar Boundary-Layer Stability and Transition on Curved Boundaries," NACA Wartime Report, pp. 1-68. 
McAuliffe, B. R. and Yaras, M. I. (2007), "Numerical study of turbulent spot development in a separated shear layer," ASME Paper No. 2007-GT-27064.

McEligot, D. M. and Jackson, J. D. (2004), "Deterioration criteria for convective heat transfer in gas flow through non-circular ducts," Nuclear Engineering and Design, 232, pp. 327-333.

Meroney, R. N. and Bradshaw, P. (1975), "Turbulent Boundary-Layer Growth over a Longitudinally Curved Surface," American Institute of Aeronautics and Astronautics, 13, No. 11, pp. 1448-1453.

Moin, P. and Mahesh, K. (1998), "Direct Numerical Simulation: A Tool in Turbulence Research,” Annual Review of Fluid Mechanics, 30, pp. 539-578.

Mokhtarzadeh-Dehghan, M. R. and Yuan, Y. M. (2002), "Measurements of turbulence quantities and bursting period in developing turbulent boundary layers on the concave and convex walls of a $90^{\circ}$ square bend," Experimental Thermal and Fluid Science, 27, pp. 59-75.

Morkovin, M. V. (1969), "Critical evaluation of transition from laminar to turbulent shear layers with emphasis on hypersonically travelling bodies," Technical Report AFFDL-TR-68-149.

Moser, R. D. and Moin, P. (1987), "The effects of curvature in wall-bounded turbulent flows," Journal of Fluid Mechanics, 175, pp. 479-510. 
Muck, K. C., Hoffmann, P. H. and Bradshaw, P. (1985), "The effect of convex surface curvature on turbulent boundary layers," Journal of Fluid Mechanics, 161, pp. 347 369.

Natesan, K., Sundararajan, T., Narasimhan, A. and Velusamy, K. (2010), "Turbulent flow simulation in a wire-wrap rod bundle of an LMFBR," Nuclear Engineering and Design, 240, pp. 1063-1072.

Ninokata, H., Merzari, E. and Khakim, A. (2009), "Analysis of low Reynolds number turbulent flow phenomena in nuclear fuel pin sub-assemblies of tight lattice configuration," Nuclear Engineering and Design, 239, pp. 855-866.

Ninokata, H. and Merzari, E. (2008), "Computer Simulation of Turbulent Flow Phenomena in Nuclear Fuel Pin Sub-assemblies," Indonesian Journal of Physics, 19, No. 1, pp. 1-11.

NIST (2011), "Thermophysical Properties of Fluid Systems," NIST Standard Reference Data, United States Department of Commerce.

Orr, W. M. F. (1907), "The stability or instability of the steady motions of a perfect liquid and of a viscous liquid," In: Proceedings of the Royal Irish Academy, 27, pp. 9138.

Ozalp, A. A. and Umur, H. (2003), "An experimental investigation of the combined effects of surface curvature and streamwise pressure gradients both in laminar and turbulent flows," Heat and Mass Transfer, 39, pp. 869-876. 
Patel, V. C. and Sotiropoulos, F. (1997), "Longitudinal Curvature Effects in Turbulent Boundary Layers," Progress in Aerospace Sciences, 33, pp. 1-70.

Peniguel, C., Rupp, I., Rolfo, S. and Guillaud, M. (2010), "Thermal-hydraulics and conjugate heat transfer calculation in a wire-wrapped SFR assembly," Proceedings of the ICAPP '10, Paper 10174, CA, USA.

Pioro, I. L. and Duffey, R. B. (2005), "Experimental heat transfer in supercritical water flowing inside channels (survey)," Nuclear Engineering and Design, 235, pp. 24072430.

Pioro, 1. L., Khartabil, H. F. and Duffey, R. B. (2004), "Heat transfer to supercritical fluids flowing in channels - empirical correlations (survey)," Nuclear Engineering and Design, 230, pp. 69-91.

Pope, S. B. (2000), “Turbulent Flows," Cambridge, UK.

Prandtl, L. (1904), "Uber Flussigkeitsbewegung bei sehr kleiner Reibung," Third International Congress of Mathematicians, Heidelberg, August 8-13. "Motion of fluids with very little viscosity," Vier Abhandlungen zur Hydrodynamik und Aerodynamik, Gottingen, 1927.

Prandtl, L. (1925), "Bericht uber untersuchungen zur ausgebildeten turbulenz," ZAMM, 5, pp. 136-139. 
Ramaprian, B. R. and Shivaprasad, B. G. (1977), "Mean Flow Measurements in Turbulent Boundary Layers along Mildly Curved Surfaces," American Institute of Aeronautics and Astronautics, 15, No. 2, pp. 189-196.

Ramaprian, B. R. and Shivaprasad, B. G. (1978), "The structure of turbulent boundary layers along curved surfaces," Journal of Fluid Mechanics, 85, pp. 273-303.

Ramaprian, B. R. and Shivaprasad, B. G. (1982), "The instantaneous structure of mildly curved turbulent boundary layers," Journal of Fluid Mechanics, 115, pp. 39-58.

Raza, W. and Kim, K. Y. (2008), "Comparative Analysis of Flow and Convective Heat Transfer between 7-pin and 19-pin Wire-Wrapped Fuel Assemblies," Journal of Nuclear Science and Technology, 45, No. 7, pp. 653-661.

Raza, W. and Kim, K. Y. (2008), "Effects of wire-spacer shape in LMR on thermalhydraulic performance," Nuclear Engineering and Design, 238, pp. 2678-2683.

Redjem-Saad, L., Ould-Rouiss, M. and Lauriat, G. (2007), "Direct numerical simulation of turbulent heat transfer in pipe flows: Effect of Prandtl number," International Journal of Heat and Fluid Flow, 28, pp. 847-861.

Robinson, S. K. (1990), "A perspective on coherent structures and conceptual models for turbulent boundary layer physics," American Institute of Aeronautics and Astronautics, Paper no. 90-1638.

Robinson, S. K. (1991), "Coherent motions in the turbulent boundary layer," Annual Review of Fluid Mechanics, 23, pp. 601-639. 
Roelofs, F. (2004), "CFD Analyses of Heat Transfer to Supercritical Water Flowing Vertically Upward in a Tube," NRG, pp. 1-28.

Rolfo, S., Peniguel, C., Guillaud, M. and Laurence, D. (2011), "Thermal-hydraulic simulations of a wire spacer fuel assembly," Nuclear Engineering and Design.

Sabatino, D. R. and Smith, C. R. (2008), "Turbulent spot flow topology and mechanisms for surface heat transfer," Journal of Fluid Mechanics, 612, pp. 81-105.

Saric, W. S., Reed, H. L. and Kerschen, E. J. (2002), "Boundary-Layer Receptivity to Free Stream Disturbances," Annual Review of Fluid Mechanics, 34, pp. 291-319.

Schlichting, H. (1979), "Boundary-Layer Theory," Seventh Edition, McGraw-Hill, USA.

Schlichting, H. (1933), "Berechnung der anfachung kleiner storungen bei der platenstromung," ZAMM, 13, pp. 171-174.

Schubauer, G. B. and Skramstad, H. K. (1947), "Laminar Boundary-Layer Oscillations and Transition on a Flat Plate," Journal of Research of the National Bureau of Standards, 38, pp. 251-292.

Singer, B. A. and Joslin, R. D. (1994), "Metamorphosis of a hairpin vortex into a young turbulent spot," Physics of Fluids, 6, No. 11, pp. 3724-3736.

Smith, C. R., Walker, J. D. A., Haidari, A. H. and Sobrun, U. (1991), "On the Dynamics of Near-Wall Turbulence," Philosophical Transactions: Physical Sciences and Engineering, 336, No. 1641, pp. 131-175. 
Shang, Z. and Lo, S. (2010), "Numerical investigation of supercritical water-cooled nuclear reactor in horizontal rod bundles," Nuclear Engineering and Design, 240, pp. 776-782.

So, R. M. C. (1975), "A turbulence velocity scale for curved shear flows," Journal of Fluid Mechanics, 70, pp. 37-57.

So, R. M. C. and Mellor, G. L. (1973), "Experiment on convex curvature effects in turbulent boundary layers," Journal of Fluid Mechanics, 60, pp. 43-62.

So, R. M. C. and Mellor, G. L. (1978), "Turbulent Boundary Layers with Large Streamline Curvature Effects," Journal of Applied Mathematics and Physics, 29, pp. 54-74.

Sommerfeld, A. (1909), "Ueber die Ausbreitung der Wellen in der drahtlosen telegraphie," Annual Physik, 28, pp. 665-737.

Söylemez, H. T. and Özkol, I. (2003), "The Stability Dependence on the Different Pressure Gradients in a Channel Flow," Aircraft Engineering and Aerospace Technology, 75, pp. 485-490.

Spalart, P. R. (1988), "Direct numerical simulation of a turbulent boundary layer up to $\operatorname{Re}_{\theta}=1410$, , Journal of Fluid Mechanics, 187, pp. 61-98.

Sreenivasulu, T. and Prasad, B. (2011), "Flow and Heat Transfer Characteristics in a Seven-Tube Bundle Wrapped with Helical Wires," International Journal of Advancements in Technology, 2, No. 3, pp. 350-381. 
Stanislas, M., Perret, L. and Foucaut, J. M. (2008), "Vortical structures in the turbulent boundary layer: a possible route to a universal representation," Journal of Fluid Mechanics, 602, pp. 327-382.

Tollmien, W. (1929), "Uber die entstehung der turbulenz," Nachr. Ges. Wiss. Gottingen, pp. 21-44.

Torgerson, D. F., Shalaby, B. A. and Pang, S. (2006), "CANDU technology for Generation III+ and IV reactors," Nuclear Engineering and Design, 236, pp. 15651572.

Umur, H. and Ozalp, A. A. (2006), "Fluid flow and heat transfer in transitional boundary layers: effects of surface curvature and free stream velocity," Heat and Mass Transfer, 43, pp. 7-15.

Winterton, R. H. S. (1998), "Where did the Dittus and Boelter equation come from?," International Journal of Heat and Mass Transfer, 41, No. 4-5, pp. 809-810.

Wu, X. and Moin, P. (2009), "Direct numerical simulation of turbulence in a nominally zero-presssure-gradient flat-plate boundary layer," Journal of Fluid Mechanics, 630, pp. 5-41.

Wu, X. and Moin, P. (2010), "Transitional and turbulent boundary layer with heat transfer," Physics of Fluids, 22, 085105, pp. 1-8. 
Yamagata, K., Nishikawa, K., Hasegawa, S., Fujii, T. and Yoshida, S. (1972), "Forced Convective Heat Transfer to Supercritical Water Flowing in Tubes," International Journal of Heat and Mass Transfer, 15, pp. 2575-2593.

Zhou, J., Adrian, R. J., Balachandar, S. and Kendall, T. M. (1999), "Mechanisms for generating coherent packets of hairpin vortices in channel flow," Journal of Fluid Mechanics, 387, pp. 353-396. 